\title{
Novel Structural Design of a Piezo Transducer for Roadway Power Harvesting Applications
}

by

\author{
Matteo J. Louter
}

A thesis submitted to the Faculty of Graduate and Postdoctoral Affairs in partial fulfillment of the requirements for the degree of

\section{Master of Applied Science}

in

Mechanical Engineering

Department of Mechanical \& Aerospace Engineering

Carleton University

Ottawa, Ontario

(C) 2015, Matteo J. Louter 


\begin{abstract}
The roadway power harvesting project was started to design and produce a working prototype of a piezoelectric based power harvester for applications in vehicle power harvesting. The system is composed of a rubber speed bump with arrays of piezoelectric transducers. The transducers are connected to power harvesting circuitry which can capture and store energy generated by the transducers and deliver it at a specific voltage to an arbitrary load application. This thesis focuses on the hardware aspect of the project, more specifically the design and fabrication and experimental validation of a novel piezoelectric transducer that incorporates both radial slits with circumferential preloading of each transducer in order to improve power generation from each transducer. The designs undergo finite element analysis for appropriate parameter determination and prototypes are tested to confirm the finite element analysis conclusions. Finally the fully constructed system is tested both on a hydraulic loading machine and with a road vehicle and determined to have a $113 \%$ improvement in peak voltage generation over a conventional cymbal transducer design with similar parameters.
\end{abstract}


To Amy.

Somewhere, something incredible is waiting to be known.

- Carl Sagan 


\section{Acknowledgements}

This research was assisted by a great many people, all of which, big or small, contributed in their own way.

This project couldn't have been completed as is without the encouragement, support, and guidance of my supervisors Professor Junjie Gu and Professor Jie Liu.

I would like to thank the help of my research partner, Xinghe, for his hard work on the circuitry component of the project as well as a great help in bouncing ideas off of and pointing out potential flaws.

A special thanks to Ebrahim Desai who sparked the project and without his support, would not have been possible.

I would also like to thank all the lab technicians and professionals who helped in many of the prototype construction and testing phases, namely Alex Proctor, Kevin Sangster, Ian Lloy, and David Raude among others.

And finally I would like to thank my parents for supporting me the whole way, and nurturing my interests in science and technology. 


\section{Contents}

1 Introduction $\quad 1$

1.1 Motivation .......................... 1

1.2 Background ............................. 2

1.2.1 Piezoelectric applications ............... 2

1.2.2 Vehicle energy harvesting .............. 4

1.2.3 Piezoelectric materials . . . . . . . . . . . . 4

1.2.4 Piezoelectric notation ............... 6

1.2.5 Piezoelectric material properties . . . . . . . . . . 11

1.3 Thesis overview . . . . . . . . . . . . . . . . 14

2 Power harvesting electronics $\quad 16$

2.1 Brief history of harvesting circuitry . . . . . . . . . . 16

2.2 The circuit model . . . . . . . . . . . . . . 18

3 Transducer design $\quad 23$

3.1 Transducer designs . . . . . . . . . . . . . . . 23

3.2 Finite element analysis model . . . . . . . . . . . . . . 29

3.2.1 Material selection ................. 33

3.2.2 Endcap thickness model . . . . . . . . . . . 36 
3.2 .3 Epoxy ooze model . . . . . . . . . . . . . . . . . 38

$3.2 .4 \quad$ FEA preload model . . . . . . . . . . . . . . . . 40

3.2.5 Analytical preload model . . . . . . . . . . . . . . 46

3.2 .6 Slit model . . . . . . . . . . . . . . . . . . . . . . 49

3.3 FEA conclusions $\ldots \ldots \ldots \ldots \ldots \ldots \ldots \ldots \ldots \ldots$

4 Implementation and testing $\quad 54$

4.1 Initial transducer designs . . . . . . . . . . . . . . . . 54

4.1 .1 Bridge transducer . . . . . . . . . . . . . . . . 54

4.1 .2 Cymbal transducer . . . . . . . . . . . . . . 54

4.2 Comparison of slitted and solid endcapped transducers . . . . . . 59

4.2 .1 Initial feasibility testing $\ldots \ldots \ldots \ldots \ldots \ldots$

$4.2 .2 \quad$ Feasibility testing results . . . . . . . . . . . . 61

$4.2 .3 \quad$ Epoxy ooze experiment . . . . . . . . . . . . . 65

4.2.4 Initial prestressed and water cut transducers . . . . . . . 68

4.2.5 Preloading of a slitted transducer . . . . . . . . . . 75

4.2.6 Transducer loading and end cap material experiment . . . . 77

4.3 Preload measurement . . . . . . . . . . . . . . . . . 81

4.4 Harvester design $\ldots \ldots \ldots \ldots \ldots \ldots \ldots \ldots \ldots$ 


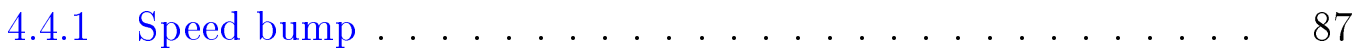

4.4 .2 Power harvesting module . . . . . . . . . . . . . . 88

4.4.3 Manufacturing of preloaded transducers . . . . . . . . 89

4.4 .4 Solid transducers . . . . . . . . . . . . . . . . . . 93

4.4.5 Assembly of the harvesting speed bump . . . . . . . . . 94

4.5 Vehicle testing . . . . . . . . . . . . . . . . 95

4.5.1 Initial vehicle test setup $\ldots \ldots \ldots \ldots \ldots \ldots$

4.5 .2 Test results $\ldots \ldots \ldots \ldots \ldots \ldots \ldots \ldots$

4.5.3 Effect of vehicle speed on power generation . . . . . . . . 103

4.5.4 Reducing preload tension . . . . . . . . . . . . . 106

4.5.5 Transducer damage . . . . . . . . . . . . . . . . . 110

$4.6 \quad$ Full scale roadway harvester . . . . . . . . . . . . . . . . . 113

4.6.1 Full scale harvester design and fabrication . . . . . . . . 113

4.6.2 Full scale harvester test . . . . . . . . . . . . . 117

5 Conclusion

5.1 Summary . . . . . . . . . . . . . . . . . . . . 124

5.2 Recommendations for future work . . . . . . . . . . . 125

References 


\section{List of Tables}

1.1 Piezoelectric properties nomenclature . . . . . . . . . . . . . 12

2.1 APC materials and their $d \cdot g$ values. . . . . . . . . . . 18

2.2 Calculated compressions needed to charge the $200 \mathrm{~F}$ capacitor pack . 21

3.1 Model default parameters . . . . . . . . . . . . . 31

3.2 Materials used and their properties. . . . . . . . . . . . 34

3.3 Model updated default parameters. . . . . . . . . . . . . . 42

4.1 Harvester MTS ${ }^{\circledR}$ machine test peak voltage results. . . . . . . . . . 59

4.2 Harvester MTS ${ }^{\circledR}$ machine test power dissipation results. . . . . . . 59

4.3 Power dissipated by the oscilloscope, produced by transducers in the 1st experiment. ......................... 64

4.4 Power produced by the rebuilt transducers. . . . . . . . . . . . . . 67

4.5 Material and load MTS $^{\circledR}$ test results. . . . . . . . . . . . . . 80

4.6 Initial vehicle test results. . . . . . . . . . . . . . 98

4.7 Peak voltage attained by transducers using reduced preloading tension. 108 


\section{List of Figures}

1.1 Piezoelectric generator modes: mode 33 (left) and mode 31 (right). .7

2.1 Capacitor storage bank. . . . . . . . . . . . . 20

3.1 THUNDER transducer manufactured by FACE ${ }^{\circledR}$ International. . . . . 24

3.2 Cymbal transducer with cutout. . . . . . . . . . . 25

3.3 Schematic of a modified electromechanical model for a preloaded Cym-

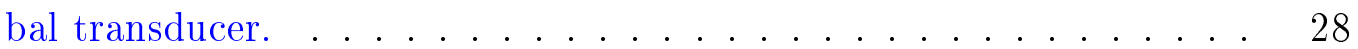

3.4 Basic axisymmetric model geometry. . . . . . . . . . . . . . 30

3.5 Parametric cross section model of a Cymbal transducer for FEA purposes. 31

3.6 FEA geometry mesh. . . . . . . . . . . . . . . . 32

3.7 Maximum calculated stress in the stainless steel end cap. $\quad$. . . . . . 37

3.8 Maximum calculated stress in the piezo disc. . . . . . . . . . . 37

3.9 Total electric energy produced under compression. . . . . . . . . . 37

3.10 Maximum stress in the end cap while varying the ooze depth. . . . . 39

3.11 Max stress in the piezo material while varying ooze depth. . . . . . . 39

3.12 Electrical energy generated as a function of ooze depth. . . . . . . . 40

3.13 Preload model FEA mesh. . . . . . . . . . . . . . . 43

3.14 Peak piezo material stress for the range of end cap thicknesses and preload tensions examined. . . . . . . . . . . . . 
3.15 Peak stress in the epoxy region of the model in a range of and cap thicknesses and preload tensions tested. . . . . . . . . . .

3.16 Peak stress in the stainless steel end cap region of the model in a range of and cap thicknesses and preload tensions. . . . . . . . . . . . 46

3.17 Analytic preload model cross section. . . . . . . . . . . . . 47

3.18 Analytic model top view. . . . . . . . . . . . . . . . . . 48

3.19 A single twelfth slice of a six slit transducer with a rough mesh for testing model stability . . . . . . . . . . . . 50

3.20 Slit model parametric study results, varying the number of slits. . . . 51

3.21 Maximum end cap stresses in the slit model plotted against end cap

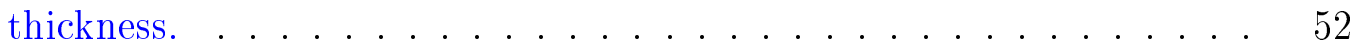

3.22 Maximum PZT stresses in the slit model plotted against end cap thick-

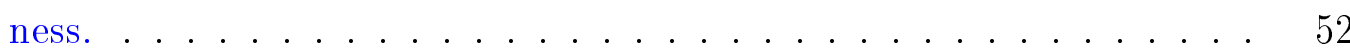

4.1 Bridge transducer. . . . . . . . . . . . . . . 54

4.2 Cymbal end cap male and female dies attached to a die set (center-left) with bridge dies (right). . . . . . . . . . . 55

4.3 Manual press for operating the die set. . . . . . . . . . 55

4.4 Early tested vehicle harvester. . . . . . . . . . . 56

$4.5 \operatorname{MTS}^{\circledR}$ test setup. . . . . . . . . . . . . . 57

4.6 Example harvester traces measured during the MTS ${ }^{\circledR}$ tests. . . . . . 58

4.7 Machined cast nylon individual transducer test housing. . . . . . . 60 
4.8 Image of a slitted (left) and solid (right) transducers assembled before wiring and testing. . . . . . . . . . . . . 61

4.9 The over performing transducer, "Slitted Transducer 2". . . . . . . . . 62

4.10 The under performing transducer (Taken apart after the test), "Slitted

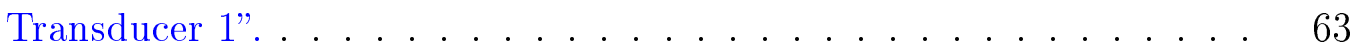

4.11 Traces of tested transducers during 1 st test. . . . . . . . . . .

4.12 One of the solid end capped transducers (Solid Transducer 1), taken

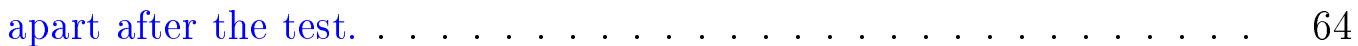

4.13 The other solid end capped transducer (Solid Transducer 2) taken apart after the test (The PZT material cracked during disassembly). . . . .

4.14 The rebuilt transducers from left to right: Slitted Transducer 2, Slitted transducer 1 , Solid traducer. . . . . . . . . . . . . .

4.15 Traces from the epoxy ooze MTS ${ }^{\circledR}$ test. . . . . . . . . . . . . 66

4.16 Slitted Transducer 1 after testing. The break seems to be caused by the small patch without epoxy near the left edge of the end cap on the

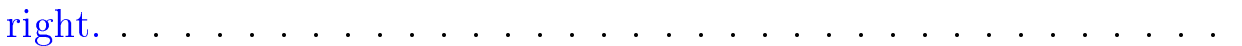

4.17 Slitted Transducer 2 after testing. The failure in this case seems to be caused by the larger lump of conductive epoxy near the top left of the left hand end cap. . . . . . . . . . . . . . . .

4.18 The solid transducer that performed very well and did not fail during the test despite small conductive epoxy ooze seen on the left end cap.

4.19 Six and four slit transducer end caps produced by water jet cutting compared to the plain versions on the right. . . . . . . . . 
4.20 Preloaded transducers next to their non-preloaded counterparts (left) and assembled water cut end cap slitted transducers (right). . . . . 70

4.21 Trace of the four slit transducer. . . . . . . . . . . . . . 70

4.22 The six slitted transducer after testing. . . . . . . . . . . 71

4.23 Preloaded transducer after $5 \mathrm{KN}$ load. . . . . . . . . . . . . 71

4.24 Failure of the preloaded cymbal transducer with $0.017 \mathrm{~J}(0.432 \mathrm{~mm})$

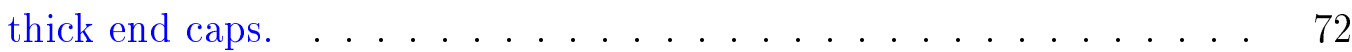

4.25 Failure of the non-preloaded cymbal transducer with $0.017 \mathrm{~J}(0.432 \mathrm{~mm})$ thick end caps. . . . . . . . . . . . . . 73

4.26 First failure of the non-preloaded, 0.013j $(0.330 \mathrm{~mm})$ transducer. . . . 74

4.27 Second failure of the non-preloaded, 0.013j $(0.330 \mathrm{~mm})$ transducer. . . 74

4.28 Failure of the preloaded cymbal transducer with $0.013 \mathrm{~J}(0.330 \mathrm{~mm})$ thick end caps. .......................... 75

4.29 The preloaded six slit transducer after testing (left) The same transducer with the testing pucks removed (right). . . . . . . . 76

4.30 Preloaded six slitted transducer trace. . . . . . . . . . . . 77

4.31 The solid end capped transducers after the MTS ${ }^{\circledR}$ test (numbered 1-4

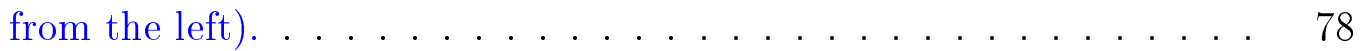

4.32 Solid transducers after testing with end caps removed (ordered 1-4 from

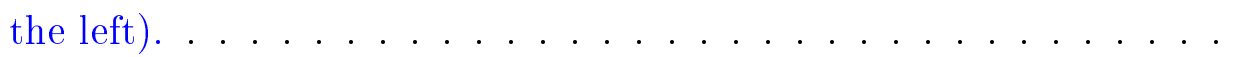

4.33 Voltage trace of transducer 1 for a sinusoidal load of 0 - $200 \mathrm{~N}$ labeled "V1-0-200" and for a load range of 0 - 250 N labeled "V1-0-250". . . . 
4.34 Fujifilm Prescale ${ }^{T M}$ sample pack. . . . . . . . . . . . . . 82

4.35 Tekscan ${ }^{\circledR}$ FlexiForce ${ }^{\circledR}$ A201 force sensor. . . . . . . . . . . . . 83

4.36 Omega ${ }^{\circledR}$ KFH-03-120-C1-11L1M2R strain gauge. . . . . . . . . 83

4.37 Omega ${ }^{\circledR}$ strain gauge mounted on a preloaded transducer. . . . . . . 84

4.38 Bridge circuit used to measure preload stress. . . . . . . . . 85

4.39 A preloaded and slitted transducer set to the correct preload. . . . . 87

4.40 Barco mini speed hump section. . . . . . . . . . . . 88

4.41 Power harvesting module design for accommodating preloaded transducers. . . . . . . . . . . . . . . . . . . 89

4.42 Power harvesting module design for standard cymbal transducers. . . 89

4.43 Slitted end caps before and after stamping. . . . . . . . . . . 90

4.44 Preloaded transducers being epoxied (left) and the hardened transduc-

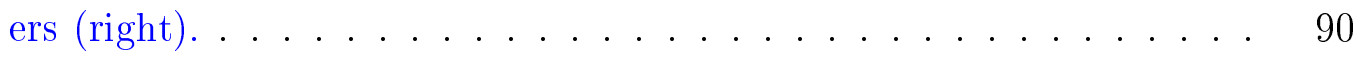

4.45 Preloaded transducers being paired up and electrically connected (left) and the finished preloaded transducers (right). . . . . . . . 91

4.46 Preloaded transducer housing after machining with inserted transducers. 92

4.47 Under performing slitted transducer. . . . . . . . . . . . . . 93

4.48 Machined non preloaded transducer housing module sitting in the speed

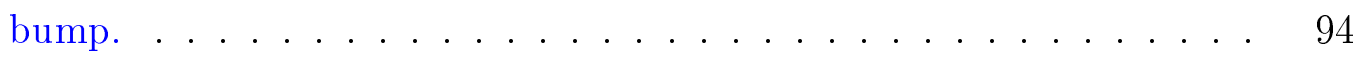

4.49 Fully assembled non preloaded harvester. . . . . . . . . . . . 94 
4.50 Harvesters inserted into speed bump. . . . . . . . . . .

4.51 Vehicle test setup. . . . . . . . . . . . . . 96

4.52 Trial 1 traces. . . . . . . . . . . . . . . . . 99

4.53 Trial 2 traces showing backward driving on the left, and forward driving on the right. . . . . . . . . . . . . . 100

4.54 Trial 3 traces. . . . . . . . . . . . . . . . . 101

4.55 Trial 7 traces showing voltage spikes corresponding to the heavier front, and lighter rear tire loads. . . . . . . . . . . . . . . . . . 102

4.56 Summary of all the peak voltages of the August vehicle testing. . . . 102

4.57 Vehicle speed test setup. . . . . . . . . . . . . 103

4.58 Peak open circuit voltages vs speed. . . . . . . . . . . . . . 104

4.59 Traces from the experimental transducers with foam padding removed. 105

4.60 Peak open circuit voltages vs speed with foam padding removed. . . . 106

4.61 Waterproofed harvesting pads. . . . . . . . . . . 107

4.62 Reduced preload traces. . . . . . . . . . . . . . 108

4.63 Short term degradation test. . . . . . . . . . . . . 109

4.64 Peak voltages plotted against speed for the reduced preload tests. . . 109

4.65 Damaged transducer traces. . . . . . . . . . . . . 111

4.66 Damaged transducer in a pair. . . . . . . . . . . 111 
4.67 Damaged piezo disc from Figure 4.66. . . . . . . . . . . . . . . 112

4.68 The second damaged transducer disassembled. . . . . . . . . . . . . 112

4.69 The most heavily damaged piezo unit examined. . . . . . . . . . . . 112

4.70 Improved harvesting pad housing design. . . . . . . . . . . . . . . 114

4.71 Transducers at various stages of assembly. . . . . . . . . . . . 114

4.72 A single harvesting pad wired and sealed with electrical tape. . . . . 115

4.73 Speed bump with truss connector plates used to attach the wooden

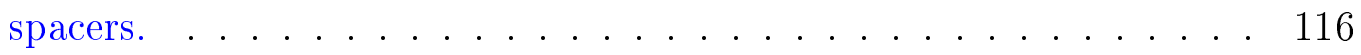

4.74 Harvesting pads on top of and placed in the speed bump. . . . . . . 116

4.75 Full scale harvester test setup. . . . . . . . . . . . . . 117

4.76 Initial compression with only harvesting pad 2. . . . . . . . . 118

4.77 Schematic for a voltage divider used to allow measurement of higher voltages. . . . . . . . . . . . . . . . . 118

4.78 Calculated full scale traces from initial front wheel loading. . . . . . . 119

4.79 All seven, $20 \mathrm{lb}$ weights placed in the rear of the test vehicle. . . . . 120

4.80 Calculated voltage traces for rear wheel loading scenarios. . . . . . . 121

4.81 Calculated peak voltages from all harvesting pads plotted against the added mass for rear wheel loading scenarios. . . . . . . . . . . 121

4.82 Vehicle speed vs average peak harvesting pad voltage. . . . . . . . 122 


\title{
Nomenclature
}

\author{
Abbreviations
}

AADT Annual Average Daily Traffic

APC American Piezo Ceramics International Ltd

CNC Computer Numeric Control

DC Direct Current

DIP Dual Inline Package

FEA Finite Element Analysis

LED Light Emitting Diode

MATLAB MATrix LABoratory

MEMS Micro Electro Mechanical Systems

MFC Micro Fiber Composites

PCB Printed Circuit Board

PVDF Polyvinylidene fluoride, a piezoelectric polymer

PZT Lead Zirconate Titanate

SONAR SOund Navigation And Ranging

THUNDER THin layer UNimorph ferroelectric DrivER and sensor

\section{Mathematical Symbols}

$\varepsilon$

Permittivity tensor $\ldots \ldots \ldots \ldots \ldots \ldots \ldots \ldots \ldots \ldots \ldots \ldots \ldots \ldots \ldots \ldots \ldots \ldots \ldots \ldots$ 
Permittivity perpendicular to polarization direction $\ldots \ldots \ldots[\mathrm{F} / \mathrm{m}]$

Piezoelectric charge constant with polarization direction $\mathrm{x}$ and strain in direction $\mathrm{y}$

A tensor that relates stress to surface charge density $\ldots \ldots \ldots\left[\mathrm{C} / \mathrm{m}^{2}\right]$

Normal force

Piezoelectric voltage constant .$[\mathrm{V} \cdot \mathrm{m} / \mathrm{N}]$

Piezoelectric charge constant with polarization direction $\mathrm{x}$ and strain in direction $\mathrm{y}$ $[\mathrm{V} \cdot \mathrm{m} / \mathrm{N}]$

$k_{p} \quad$ Electromechanical coupling factor, used for thin discs, with electric field in direction 3 and radial vibrations in directions 1 and $2 \ldots[\%]$ 
$k_{p}^{2} \quad$ Electromechanical coupling factor for a thin piezoelectric disc being stressed at static or low frequencies

$k_{t} \quad$ Electromechanical coupling factor used for thin discs $\ldots \ldots \ldots[\%]$

$k_{x y} \quad$ Electromechanical coupling factor with electric field in direction $\mathrm{x}$ and

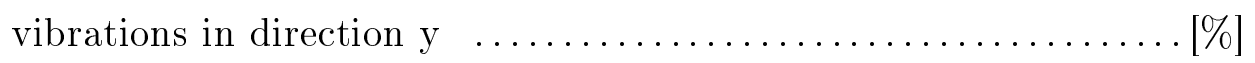

$K_{x y}^{T} \quad$ Relative dielectric constant, at a constant force in direction $\mathrm{y}$, with polarization in direction $\mathrm{x}$

$N$

$Q$

$s$

$s^{E}$

$s_{11}^{E}$

$s_{33}^{D}$

u

V

$V_{c}$

$V_{r t}$

$W$

$X$
Number of radial wedges

Generated charge

Elastic compliance $\left[\mathrm{m}^{2} / \mathrm{N}\right]$

Compliance under a constant electric field $\left[\mathrm{m}^{2} / \mathrm{N}\right]$

Elastic compliance perpendicular to polarization direction, connected in short circuit $\left[\mathrm{m}^{2} / \mathrm{N}\right]$

Elastic compliance parallel to polarization direction, connected in open circuit $\left[\mathrm{m}^{2} / \mathrm{N}\right]$

Displacement $[\mathrm{m}]$

Voltage

Static voltage of a piezoelectric ring under compression

Static voltage of a piezoelectric disc under radial tension

Work

Mechanical strain 
Mechanical stress

Young's modulus $[\mathrm{Pa}]$

Inside diameter parameter $[\mathrm{m}]$

Outside diameter parameter $[\mathrm{m}]$

Thickness parameter $[\mathrm{m}]$ 


\section{Introduction}

This work covers the motivation, background, design, and testing of a piezoceramic based power harvesting system designed to harvest power from vehicles. The final goal of this project is to build working prototypes of a roadway power harvesting system to be implemented in either speed bumps, or in the road surface itself. Using the aforementioned piezoelectric technology, the speed bump would be able to store and power a variety of applications through on board power harvesting circuitry and power conditioning circuitry to deliver the power at a variety of voltages. The number of speed bumps or harvesting pads, the number of power harvesting transducers per pad, and the electrical storage capacity would be determined by the power demanded by a specific application.

\subsection{Motivation}

As more people become aware of the effects of global warming, there is greater commercial pressure to develop sustainable energy technologies. One area that has attracted some attention is roadway power harvesting using piezoelectric materials [1]. Power harvesting is not only attractive for its use of so called green energy but is also useful for replacing batteries as power sources where applicable with the added benefit of not needing replacement [2]. Finally a very large proportion of energy that is used by vehicles is given off in the form of waste heat from braking and road deformation

$[3,4]$. This energy can be recovered for other purposes by placing roadway power 
harvesters on roadways, in particular areas of vehicle deceleration or where power is needed.

In addition to the stated goals, automated speed measurement is also explored for harvesting pads with two or more piezoelectric harvesting arrays. Although not part of the objectives, speed measurement would make an interesting additional feature to any future product that may come from this research.

Pavegen is a company based in the United Kingdom, striving to produce an energy harvester that is economically viable on a large scale and is producing a series of pedestrian footstep harvesting pads that use a mechanism to apply stress to piezoelectric materials [5]. Another Israeli company Innowattech is also developing piezoelectric power harvesters using simple rods of PZT [6] for applications in roadway power harvesting, railway power harvesting, and airport runway power harvesting [7, 8].

Currently there are few commercial roadway power harvesting technologies in existence, and those that do exist have not been implemented on a large scale. Because of this, BW Services and MITACS funded this research to produce a roadway power harvesting device.

\subsection{Background}

This section provides background information on piezoelectric materials, power harvesting projects and applications as well as research performed in this area. Fundamental governing equations for piezoelectric materials are also covered.

\subsubsection{Piezoelectric applications}

Piezoelectric materials are widely used today in a variety of applications. From pressure sensors, accelerometers, microphones, SONAR and ultrasound systems all the 
way to gas lighters, buzzers and clocks, piezoelectric materials are used in many aspects of daily life $[9,10,11,12,13,14,15,16]$. Piezoelectric energy harvesting has been applied to many situations that involve vibrations and where it is difficult to attach fixed power cables such as ocean buoys, knapsack straps, and shoes $[17,18,19,20,21]$.

Due to its uniform crystal nature, Piezoelectric materials allow Micro-Electro-Mechanical Systems (MEMS) such as accelerometers and clock oscillators to be made cheaply and in very small packages. Piezoelectric accelerometers work by attaching small masses through piezoelectric pads to a base fixed to the sensor body. When the sensor body accelerates, the small internal mass will put the piezo element under stress, generating a proportional voltage to the acceleration. Quartz crystal clocks use specific configurations of quartz crystals, usually shaped as thin plates or tuning forks, and include them as a component of an electronic oscillator. The quartz crystals help produce very stable oscillation frequencies and thereby keep accurate time. Piezoelectric SONAR and ultrasound systems take advantage of the inverse piezoelectric effect, and are able to produce powerful sound waves at much higher frequencies than conventional speakers by replacing the coil actuator with a piezoelectric one. High voltages can be reached when striking piezo materials, paving way for the existence of reliable and small gas lighters without the need for batteries or other external power sources. Piezoelectric gas lighters use a mechanism to store energy in a spring, which is compressed by a user, until it is fully compressed. This causes the spring to release, striking a mass into the piezoelectric element which in turn generates a high voltage and a visible spark. Often pressure sensors will use thin film piezoelectric materials spread over a small hole exposed to an external pressure. This method of pressure sensing allows for large pressures to be measured at high accuracy and even allows for absolute pressure measurement. 


\subsubsection{Vehicle energy harvesting}

Apart from piezoelectric based roadway power harvesting, research has been put into power recovery from vehicles using permanent magnet linear generators as discussed and demonstrated in [22] and hydraulic systems which are demonstrated in [3]. However most research appears to employ piezoelectric materials for power harvesting due to the lack of moving parts required and compact sizing, allowing for easier design, installation, and maintenance of power harvesting roads and pads [1, 4, 23, 24, 25, 26]

\subsubsection{Piezoelectric materials}

Piezoelectric materials generate an electric potential by way of electric field generation when stressed, called the piezoelectric effect. The inverse piezoelectric effect is also possible by way of inducing a strain in the material after an electric potential is applied.

There are a wide variety of piezoelectric materials, all well suited to various applications $[2,9,27]$. Natural and synthetic quartz crystals, a crystalline form of silicon dioxide, are an example of a non ferroelectric piezoelectric material. Generally non ferroelectric materials exhibit poorer piezoelectric properties but are still useful for applications as sensors such as pressure, gyroscopic, and microphone sensors. The largest use of quartz is as a resonator used for timing circuits in clocks, computer processors, and transmitter/receivers, to name a few.

Piezoelectric polymers are another family of piezoelectric materials geared toward their own set of applications. These polymers can be manufactured at lower temperatures and offer high flexibility and mechanical stability. With poor piezoelectric properties, these polymers are poorly suited for actuators but can work well as sensors. Among the few known piezoelectric polymers, Polyvinylidene Fluoride (PVDF) 
is one of the most widely used. Applications include vibration dampening actuators and artificial muscles. PVDF has even been experimentally used in shoes [2, 21]. Lead $(\mathrm{Pb})$ Zirconate Titanate $(\mathrm{PZT})$ is a ceramic and ferroelectric piezoelectric material which has excellent piezoelectric properties sometimes hundreds of times higher than quartz [28]. It is commonly used in transducers and actuators because of its piezoelectric and mechanical properties. PZT is a synthetic crystal with chemical formula $\operatorname{PbZr}(\mathrm{Ti}) \mathrm{O}_{3}$ in a perovskite structure. For thin films, PZT can be manufactured by chemical vapor deposition however for transducer and actuator applications where thicker solids are desired another technique is used. Powder components of the PZT crystal are combined with each other and a polymer binder in a mold and compressed under high heat. After the molding process is complete, the PZT components are fired in order to finalize the sintering process. Finally a large electric field is applied to set the polarity direction while the material is held above its Curie temperature. Without the polarization process, the PZT crystals would produce no net electric field when the material is strained. The specific properties of a PZT material can be modified by changing the zirconia to titania ratio of the precursor powders [28]. Because of these benefits, PZT is the chosen material for the piezoelectric power harvesting project presented here.

PZT is popular because of its piezoelectric properties, however being a ceramic causes that material to be brittle and therefore unsuited to many applications. In order to get the desired material properties, piezoelectric composites have been created which are called Micro Fiber Composites (MFC). Three main piezo composites, typically using PZT and a binding polymer such as epoxy, exist: $0-3,1-3$, and $3-3$. The first digit represents the number of directions that the piezoelectric material is connected in and the second represents the directions in which a binding polymer is connected in. 0-3 composites are formed by evenly dispersing powdered PZT within a polymer. Composites composed of PZT filaments suspended in a polymer are classed as 1-3 
and finally larger chunks and networks of PZT in suspension is referred to as a 33 composite. Even though these composites have improved strength characteristics compared to ceramic PZT components, the reduced amount of piezoelectric material also reduces the overall charge available to be harvested in compression. For this reason these materials were not used in this research project.

\subsubsection{Piezoelectric notation}

Piezoelectric materials have the ability to convert mechanical energy into electric charge or to convert electric charge into mechanical energy. The process of converting mechanical into electrical energy is called the piezoelectric effect [2, 11, 20, 29]. Conversely, the process of converting electrical into mechanical energy is called the inverse or converse piezoelectric effect. Because piezoelectric materials are anisotropic a common vector notation seen in literature and industrial purposes is Voigt notation $[11,30]$. This notation replaces standard right hand coordinate directions $\mathrm{x}, \mathrm{y}, \mathrm{z}$ and rotations about $\mathrm{x}, \mathrm{y}$, and $\mathrm{z}$ with the digits 1 - 6. Piezoelectric materials that are deformed in different directions will behave differently because of their anisotropy. Piezoelectric generator modes are the term used to refer to piezoelectric loading directions and their calculations. Figure 1.1 demonstrates two common modes, mode 33 on the left, and mode 31 on the right. The first digit refers to the poling direction represented in Figure 1.1 by the vector marked P. This is the direction that an electric field was applied when the material was being manufactured and is conventionally

aligned with the $\mathrm{Z}$ axis. The second digit refers to the loading direction which an applied force is aligned to. Most piezoelectric materials that are symmetric about the $\mathrm{X}-\mathrm{Z}$ plane will have properties that are equal in 31 mode and 32 mode. 


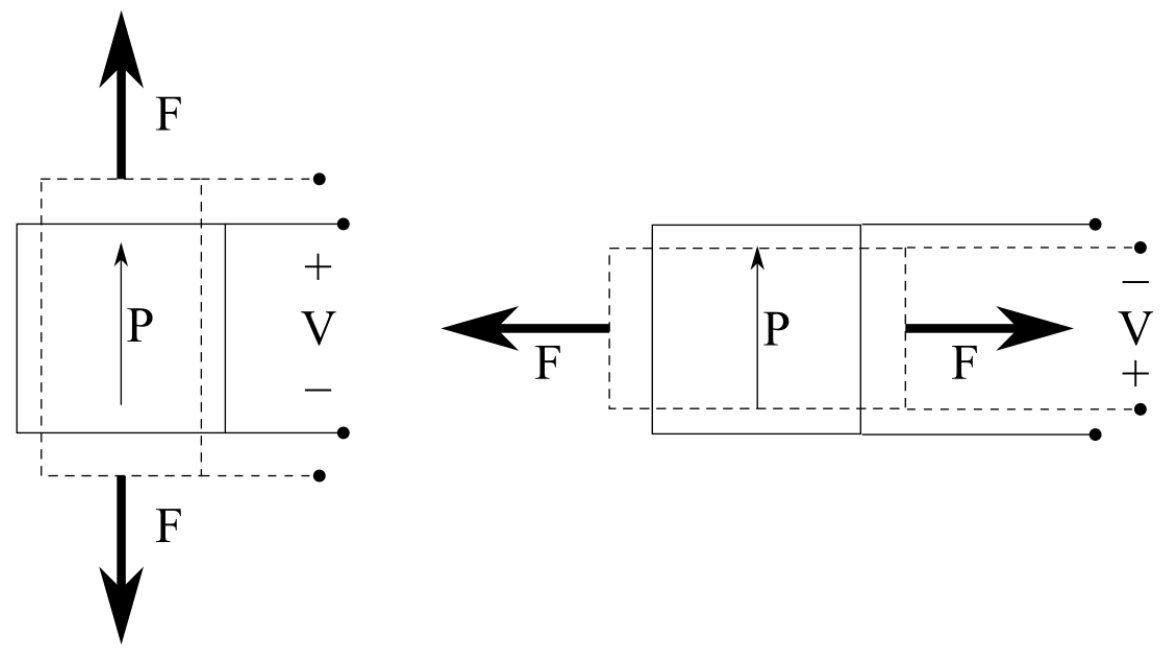

Fig. 1.1: Piezoelectric generator modes: mode 33 (left) and mode 31 (right).

The following equations model the behavior of piezoelectric materials [28]. Surface charge density and electric field are proportional to mechanical strain as described in Equations 1.1 and 1.2.

$$
\begin{aligned}
& D=d x \\
& E=g x
\end{aligned}
$$

Where $D$ is the surface charge density, $E$ is the electric field, and $x$ is the input mechanical stress. Here $d\left(\frac{C}{N}\right)$ and $g\left(\frac{V \cdot m}{N}\right)$ are piezoelectric constants unique for every piezoelectric material and often refered to as the piezoelectric charge and voltage constants respectively. The stress, $x$, is a second order tensor of the form:

$$
x=\left[\begin{array}{lll}
x_{11} & x_{12} & x_{13} \\
& x_{22} & x_{23} \\
\text { sym } & & x_{33}
\end{array}\right] .
$$


Since $x$ has six independent values, it is often rewritten in Voigt notation as a vector $[2,9,30,31]:$

$$
x=\left[\begin{array}{l}
x_{11} \\
x_{22} \\
x_{33} \\
x_{23} \\
x_{13} \\
x_{12}
\end{array}\right]=\left[\begin{array}{l}
x_{1} \\
x_{2} \\
x_{3} \\
x_{4} \\
x_{5} \\
x_{6}
\end{array}\right] .
$$

Similarly $D$ is a three component vector:

$$
D=\left[\begin{array}{l}
D_{1} \\
D_{2} \\
D_{3}
\end{array}\right]
$$

Equation 1.1 can then be rewritten as:

$$
\left[\begin{array}{c}
D_{1} \\
D_{2} \\
D_{3}
\end{array}\right]=\left[\begin{array}{llllll}
d_{11} & d_{12} & d_{13} & d_{14} & d_{15} & d_{16} \\
d_{21} & d_{22} & d_{23} & d_{24} & d_{25} & d_{26} \\
d_{31} & d_{32} & d_{33} & d_{34} & d_{35} & d_{36}
\end{array}\right]\left[\begin{array}{c}
x_{1} \\
x_{2} \\
x_{3} \\
x_{4} \\
x_{5} \\
x_{6}
\end{array}\right] .
$$

We can see that the piezoelectric constant $d$ can be represented as a $3 \times 6$ matrix. Strain $X$ is also a second order tensor like the stress tensor $x$ and can be written 
similarly to $x$ in Equations 1.3 and 1.4. $X$ can then be written in terms of $x$ by the elastic compliance constant $s$ shown in Equation 1.7.

$$
\left[\begin{array}{l}
X_{1} \\
X_{2} \\
X_{3} \\
X_{4} \\
X_{5} \\
X_{6}
\end{array}\right]=\left[\begin{array}{llllll}
s_{11} & s_{12} & s_{13} & s_{14} & s_{15} & s_{16} \\
s_{21} & s_{22} & s_{23} & s_{24} & s_{25} & s_{26} \\
s_{31} & s_{32} & s_{33} & s_{34} & s_{35} & s_{36} \\
s_{41} & s_{42} & s_{43} & s_{44} & s_{45} & s_{46} \\
s_{51} & s_{52} & s_{53} & s_{54} & s_{55} & s_{56} \\
s_{61} & s_{62} & s_{63} & s_{64} & s_{65} & s_{66}
\end{array}\right]\left[\begin{array}{l}
x_{1} \\
x_{2} \\
x_{3} \\
x_{4} \\
x_{5} \\
x_{6}
\end{array}\right]
$$

The surface charge density and electric field can be related through the permittivity tensor defined as:

$$
\left[\begin{array}{l}
D_{1} \\
D_{2} \\
D_{3}
\end{array}\right]=\left[\begin{array}{lll}
\varepsilon_{11} & \varepsilon_{12} & \varepsilon_{13} \\
\varepsilon_{21} & \varepsilon_{22} & \varepsilon_{23} \\
\varepsilon_{31} & \varepsilon_{32} & \varepsilon_{33}
\end{array}\right]\left[\begin{array}{l}
E_{1} \\
E_{2} \\
E_{3}
\end{array}\right] .
$$

Because the piezoelectric material, PZT, being used is in a crystalline structure and is poled in such a way to make it symmetric about the single poled axis (conventionally assumed to be the z-axis or direction 3) the Equations 1.6, 1.7, and1.8 can be simplified and rewritten using Equations 1.9, 1.10, and 1.11.

$$
[d]=\left[\begin{array}{cccccc}
0 & 0 & 0 & 0 & d_{15} & 0 \\
0 & 0 & 0 & d_{15} & 0 & 0 \\
d_{31} & d_{31} & d_{33} & 0 & 0 & 0
\end{array}\right]
$$

where $d_{15}=d_{51}$ and $d_{13}=d_{31}$ because of symmetry. $s$ becomes: 


$$
[s]=\left[\begin{array}{cccccc}
s_{11} & s_{12} & s_{13} & 0 & 0 & 0 \\
s_{12} & s_{11} & s_{13} & 0 & 0 & 0 \\
s_{13} & s_{13} & s_{33} & 0 & 0 & 0 \\
0 & 0 & 0 & s_{44} & 0 & 0 \\
0 & 0 & 0 & 0 & s_{44} & 0 \\
0 & 0 & 0 & 0 & 0 & 2\left(s_{11}-s_{12}\right)
\end{array}\right]
$$

where $s_{11}=s_{22}, s_{12}=s_{21}, s_{13}=s_{31}, s_{44}=s_{55}$, and $s_{66}=2\left(s_{11}-s_{12}\right)$. And finally $\varepsilon$ becomes:

$$
[\varepsilon]=\left[\begin{array}{ccc}
\varepsilon_{1} & 0 & 0 \\
0 & \varepsilon_{2} & 0 \\
0 & 0 & \varepsilon_{3}
\end{array}\right]
$$

where $\varepsilon_{1}=\varepsilon_{2}$. The governing equations for the piezoelectric effect then take on two forms based on boundary conditions. In the case where the piezoelectric material is free to expand and contract along with changing electric field the governing equation becomes:

$$
D=d x+\varepsilon E .
$$

In the case where the piezoelectric material is clamped, the equation becomes:

$$
D=e X+\varepsilon E
$$

where $e$ is the tensor that relates the stress to the surface charge density. 
The indirect piezoelectric effect can also be described using the following equations. In the case were the piezoelectric material is under a constant electric field, which corresponds to the material in a short circuit, the governing equation becomes:

$$
\begin{aligned}
& X=s^{E} x+d E, \\
& x=c^{E} X-e E,
\end{aligned}
$$

where $s^{E}$ and $c^{E}$ are the compliance and stiffness under a constant electric field. In the other case, where the piezoelectric material is in a constant charge density, corresponding to the material connected in open circuit. The equations then become:

$$
\begin{aligned}
& X=s^{D} x+g D \\
& x=c^{D} X-h D .
\end{aligned}
$$

where $h$ is the electric field strength per unit strain.

\subsubsection{Piezoelectric material properties}

Piezoelectric materials are anisotropic and so the notation used allows subscripts to define the poling and loading directions that the specific property applies in. 
Table 1.1: Piezoelectric properties nomenclature

\begin{tabular}{|c|c|}
\hline Symbol & Description \\
\hline$d_{x y}$ & $\begin{array}{l}\text { Piezoelectric charge constant with polarization direction } \mathrm{x} \text { and strain } \\
\text { in direction } \mathrm{y}\end{array}$ \\
\hline$g_{x y}$ & $\begin{array}{l}\text { Piezoelectric voltage constant with electric field in direction } \mathrm{x} \text { and } \\
\text { strain in direction } \mathrm{y}\end{array}$ \\
\hline$\varepsilon_{11}^{T}$ & Permittivity perpendicular to polarization direction \\
\hline$\varepsilon_{33}^{T}$ & Permittivity parallel to polarization direction \\
\hline$s_{11}^{E}$ & $\begin{array}{l}\text { Elastic compliance perpendicular to polarization direction, connected } \\
\text { in short circuit }\end{array}$ \\
\hline$s_{33}^{D}$ & $\begin{array}{l}\text { Elastic compliance parallel to polarization direction, connected in } \\
\text { open circuit }\end{array}$ \\
\hline$k_{x y}$ & $\begin{array}{l}\text { Electromechanical coupling factor with electric field in direction } \mathrm{x} \text { and } \\
\text { vibrations in direction } \mathrm{y}\end{array}$ \\
\hline$k_{t}$ & Electromechanical coupling factor used for thin discs \\
\hline$k_{p}$ & $\begin{array}{l}\text { Electromechanical coupling factor, used for thin discs, with electric } \\
\text { field in direction } 3 \text { and radial vibrations in directions } 1 \& 2\end{array}$ \\
\hline$Q$ & Generated charge \\
\hline$K_{x y}^{T}$ & $\begin{array}{l}\text { Relative dielectric constant, at a constant force in direction } \mathrm{y} \text {, with } \\
\text { polarization in direction } \mathrm{x}\end{array}$ \\
\hline
\end{tabular}

The piezoelectric properties in Table 1.1 can be related to each other and other physical properties using the following equations [2, 11, 28]. Equation 1.18 relates the elastic compliance to the young's modulus of the material.

$$
\begin{gathered}
s_{x y}=\frac{1}{Y_{x y}} \\
k_{p}^{2}=\frac{2 d_{31}^{2}}{\left(\left(s_{11}^{E}+s_{12}^{E}\right) \varepsilon_{33}^{T}\right)}
\end{gathered}
$$


Equation 1.19 describes the electromechanical coupling factor for a thin piezoelectric disc being stressed at static or low frequencies.

$$
\begin{gathered}
g_{x y}=\frac{d_{x y}}{\varepsilon_{x y}^{T}} \\
V=\frac{g_{x y} F t}{A}
\end{gathered}
$$

Equation 1.20 relates the piezoelectric voltage constant to the piezo charge constant. While Equation 1.21 returns the static voltage of a piezo material under load in the $y$ direction, and polarization in the $x$ direction. In this equation, $F$ is an applied force to the piezo unit, $t$ is the thickness along which the force is being applied, and $A$ is the cross sectional area perpendicular to the direction of applied force. The forces applied to the piezoelectric disc in any cymbal style transducer are a combination of compression of the outer ring, and a radial tension, both forces being applied by the end cap. These two effects can be superimposed on each other [9, 32]. Equation 1.22 defines the capacitance of a piezo disc, where $h$ is the thickness of the disc, and $r$ is the radius.

$$
\begin{gathered}
C_{s}=\frac{K_{33}^{T} \varepsilon_{0} \pi r^{2}}{h} \\
V_{r t}=\frac{g_{31} F_{r}}{2 \pi r} \\
V_{c}=\frac{4 g_{33} F_{3} h}{\pi\left(O D^{2}-I D^{2}\right)}
\end{gathered}
$$


where $O D$ and $I D$ are the outside and inside diameters defining the ring shaped region of the piezoelectric disc being compressed. Equations 1.23 and 1.24 are the static voltages of a piezoelectric disc under radial tension, and compressed piezo ring respectively. By using equations 1.22, 1.23, and 1.24, the total energy harvested from a given force can be estimated. Conversely it may be possible to use the above equations to estimate vehicle weight and transducer performance separately.

\subsection{Thesis overview}

Chapter 2: Power harvesting electronics

This chapter covers information on various piezoelectric power harvesting circuitry topologies as well as feasibility calculations using the circuitry concepts to produce an estimate of the amount of power that could be generated from a set number of harvesters. This estimated power is compared to a potential application to conclude that the estimated energy generation amount is sufficient for the potential application.

\section{Chapter 3: Transducer design}

This chapter focuses on the physical design of transducers, giving a background to other transducer designs made along with their applications. A novel variant of the Cymbal transducer designed for vehicle power harvesting is also introduced along with Finite Element Analysis (FEA) models. FEA is used to examine existing designs and to assist in finding improved design parameters for improved performance.

Chapter 4: Implementation and testing 
This chapter documents the generations of prototyped transducers and what was learned from them. It also discusses the manufacturing processes and implementation of the transducers into nylon housings to be implanted into rubber speed bumps. Results from both MTS hydraulic testing apparatus and vehicle tests are also presented here.

Chapter 5: Conclusion

Finally this chapter summaries the conclusions and accomplishments that this study found along with promising avenues for improvements and future work. 


\section{Power harvesting electronics}

This section covers various methods for piezoelectric charge collection in order to store and deliver the harvested power to lower voltage, higher current applications.

\subsection{Brief history of harvesting circuitry}

As Piezoelectric materials become stressed by an external load, an electric field is generated by the material. Often, fabricated piezoceramic solids, such as PZT, will have two sides coated in a thin conductive film. These conductive film panels behave like a capacitor allowing the generated electric field to draw in or push out electrons from the conductive contact areas. This flow of charge is the source of electrical energy to be harvested or used by circuitry or an electrical load.

The simplest way of utilizing piezoelectric power is by simply connecting the two contact terminals of a piezoelectric material to an electrical load. Many papers focusing on transducer design will report voltages attained while attaching a simple resistive load to a piezo transducer $[2,19,25,33,34]$. This method for harvesting power by direct connection to a load is the least efficient method. This method also generates inverting and often high voltages and low currents which make them difficult to adapt to lower voltage and higher direct current loads such as LED lighting or low power sensors [35].

A simple solution to the above problems is by rectifying the piezo harvesters output, adding a storage capacitor, and then using a DC - DC step down converter to produce

the desired voltage and current for a specific application. Typically the DC - DC converter chosen is a buck converter. Modeling the piezoelectric material subjected to strain can be done by representing the material as a current source with an internal 
capacitance caused by the electrodes [35]. Because of this, it is beneficial to allow the piezo material to reach a higher voltage and then step the voltage down.

More advanced harvesting circuits tend to improve power harvested by inverting or charging the internal capacitance of a harvester using an inductor [2, 33, 34, 35, 36, 37]. One way to grasp the reasoning behind inverting or pre-charging the internal piezo capacitance is by examining the work that the load force must do in order to allow the full compression of a piezoceramic or piezoceramic device. The total work done by an external force is shown in Equation 2.1 .

$$
W=F_{n o r m} \cdot d
$$

Where $W$ is the work done by an external force, $F_{n o r m}$ which is the normal force resisting the external force, and $d$ is the displacement of the compressed piezo unit under the external load. The total work can then be increased by increasing the normal force required to compress a piezoelectric transducer, assuming that the external force is sufficient to compress the transducer to its maximum allowed displacement. The stiffness of any piezoelectric material is determined by the superposition of its mechanical stiffness along with the electric field within the material due to the inverse piezoelectric effect [9]. Thus a circuit that inverts or applies an initial charge to a piezo material just before it is loaded will allow the external load to generate more work, assuming that it compresses the piezoelectric transducer a fixed amount, and therefor increase the amount of energy available for harvesting.

These circuits are referred to as synchronous circuits and are similar to the one that will be used with this project. 


\subsection{The circuit model}

The harvesting circuit being used for this project synchronizes the discharge of the piezoelectric materials with their peak voltage. As the piezoelectric material approaches its peak voltage caused by its peak strain, a peak detection circuit triggers the harvesting circuit to drain the piezoelectric capacitor into a storage capacitor. This means that the energy gathered can be approximated by Equation 2.2 where $V$ is the peak voltage of the piezoelectric material. This equation ignores losses in the circuitry, dissipation from measurement equipment, and the voltage of the storage capacitor.

$$
E=\frac{1}{2} C V^{2}
$$

A variety of possible piezoceramic materials exist for use in power harvesting applications. American Piezo Ceramics (APC) is a company that provides custom fabricated piezoelectric materials as well as testing samples. Their materials were considered because of the ease of purchasing samples from them which suited the initial testing phases of the project. A variety of APC materials are shown in Table 2.1.

Table 2.1: APC materials and their $d \cdot g$ values.

\begin{tabular}{|c|c|c|c|}
\hline Material (navy type equivalent) & $d_{31} \cdot g_{31}$ & $d_{33} \cdot g_{33}$ & $d_{15} \cdot g_{15}$ \\
\hline \hline $840($ Navy I) & 1.38 & 7.69 & 18.2 \\
\hline 841 & 1.14 & 7.65 & 15.8 \\
\hline $850($ Navy II) & 2.17 & 9.92 & 21.2 \\
\hline 855 (Navy VI) & 2.48 & 13.2 & 19.4 \\
\hline 880 (Navy III) & 0.950 & 5.38 & 9.24 \\
\hline 842 & - & 7.89 & - \\
\hline 844 & - & 7.35 & - \\
\hline 851 & - & 9.92 & - \\
\hline 881 & - & 5.87 & - \\
\hline \multicolumn{2}{|c|}{ Units in $\left(\times 10^{-12} \frac{\mathrm{ms}^{2}}{k g}\right)$} \\
\end{tabular}


APC International Ltd 855 material was chosen for its high $d_{33} \cdot g_{33}$ and $d_{31} \cdot g_{31}$ values, which represent one of the more desirable metrics for a PZT harvesting transducer [20, 28, 38]. Several PZT samples made of APC 855 were purchased with readily available dimensions. The sample discs had a diameter of $25.4 \mathrm{~mm}$ and a thickness of $2 \mathrm{~mm}$. The capacitance can be estimated for the disc using Equation 1.22:

$$
C_{s}=\frac{3,300\left(8.85 \times 10^{-12}\right) \pi 0.0127^{2}}{0.002}=6.73 \mathrm{nF} .
$$

A Tektronix DMM4050 multimeter was used to measure the capacitance of the PZT disc. The measured capacitance from the probes was $0.035 \mathrm{nF}$ and the measured capacitance of a piezo unit was $7.94 \mathrm{nF}$. Equivalently then, the capacitance of the piezo unit should be the difference of these, being $7.91 \mathrm{nF}$. The measured value is about $18 \%$ larger than the calculated value and is probably due to variations between the materials actual properties, and the reported properties. To represent the piezoelectric material more accurately, the measured value will be used in calculations.

We are using a synchronous circuit that will empty the transducer voltage into a storage capacitor when the transducer reaches its peak voltage. Because the charge is transferred from the piezoelectric power bank into a storage capacitor, not all the charge can be transferred, and so the energy transferred should be:

$$
\begin{gathered}
E_{\text {transfer }}=E_{\text {total }}-E_{\text {leftOver }}, \\
E_{\text {transfer }}=\frac{1}{2} C_{\text {piezo }} V_{\text {piezo }}^{2}-\frac{1}{2} C_{\text {piezo }} V_{\text {storage }}^{2},
\end{gathered}
$$


where $E_{\text {transfer }}$ is the total energy being transferred over from a piezo transducer, $E_{\text {total }}$ is the total energy stored in the transducer at its peak voltage, and finally $E_{\text {leftOver }}$ is the leftover energy stored in the transducer after the synchronous circuit has discharged $E_{\text {transfer }}$ into the storage capacitor bank assuming that the storage capacitance is much larger than the capacitance of the transducer. The size of storage capacitor would vary from power harvesting application to power harvesting application, however if we assume that storage capacitors used are a pair of $400 \mathrm{~F}, 2.7 \mathrm{~V}$ super capacitors. The super capacitors are shown in Figure 2.1 connected in series such that they have an equivalent capacitance and voltage limit of $200 \mathrm{~F}$, and $5.4 \mathrm{~V}$ respectively.

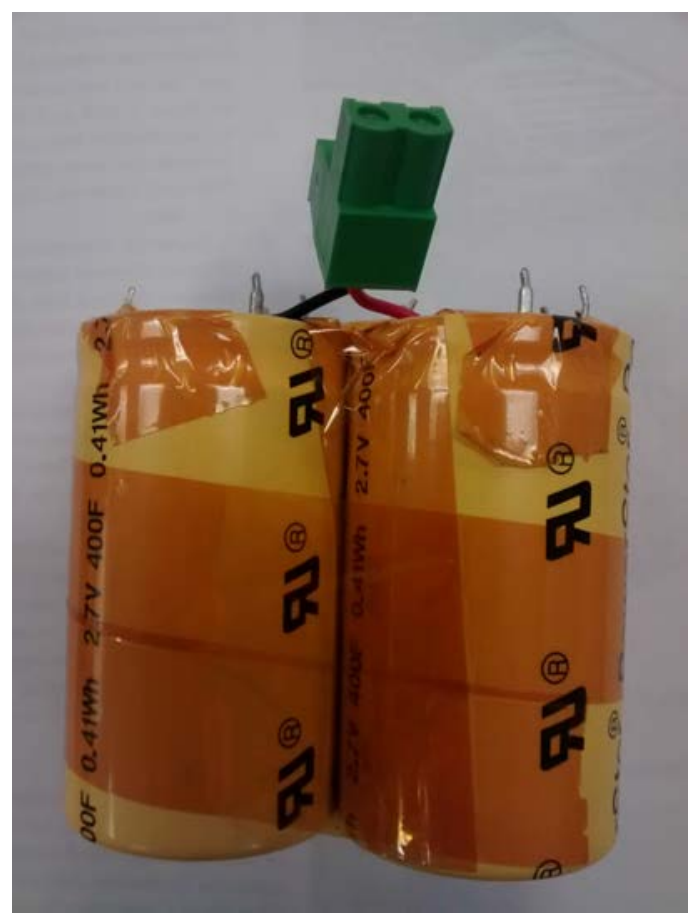

Fig. 2.1: Capacitor storage bank.

Using MATLAB ${ }^{\circledR}$, we can calculate the number of compressions needed for an arbitrary harvesting pad assuming it reaches an average peak voltage of $300 \mathrm{~V}$ and using the formula below. These equations are used to update the stored voltage after each compression: 


$$
\begin{gathered}
V_{\text {storageNew }}=\sqrt{\frac{2}{C_{\text {storage }}}\left(E_{\text {transfer }}+E_{\text {storage }}\right)} \\
V_{\text {storageNew }}=\sqrt{\frac{2}{C_{\text {storage }}}\left(\frac{1}{2} C_{\text {piezo }} V_{\text {piezo }}^{2}-\frac{1}{2} C_{\text {piezo }} V_{\text {storageOld }}^{2}+\frac{1}{2} C_{\text {storage }} V_{\text {storageOld }}^{2}\right)} \\
V_{\text {storageNew }}=\sqrt{\frac{C_{\text {piezo }}\left(V_{\text {piezo }}^{2}-V_{\text {storageOld }}^{2}\right)+C_{\text {storage }} V_{\text {storageOld }}^{2}}{C_{\text {storage }}}}
\end{gathered}
$$

Iterating the above equations in MATLAB $^{\circledR}$, the program calculates the results shown in Table 2.2. Note that the calculation iteratively added optimal amounts of energy from the piezo units until the goal voltage was met or exceeded. As such the calculated number of compressions shown in Table 2.2 are rounded up to the nearest whole number of compressions. The circuit that was used is allowed to store no more than 5 volts, and produces variable voltage power for a given load.

Table 2.2: Calculated compressions needed to charge the $200 \mathrm{~F}$ capacitor pack

\begin{tabular}{|c|c|c|}
\hline Storage voltage increase & $\begin{array}{c}2 \text { harvesting pads } \\
\text { (number of } \\
\text { compressions) }\end{array}$ & $\begin{array}{c}8 \text { harvesting pads } \\
\text { (number of } \\
\text { compressions) }\end{array}$ \\
\hline \hline $0-5 \mathrm{~V}$ & 175,611 & 43,903 \\
\hline $4-5 \mathrm{~V}$ & 63,226 & 15,807 \\
\hline $0-4 \mathrm{~V}$ & 112,386 & 28,097 \\
\hline
\end{tabular}

The $0-5 \mathrm{~V}$ trial indicates the fully discharged module being charged to maximum operating voltage, $4-5 \mathrm{~V}$ represents the harvesting module charging between minimum and maximum operating voltages and is the intended continuous mode of operation, and $0-4 \mathrm{~V}$ represents the charging to the minimum operating voltage. This system can be scaled up linearly to provide more power by simply adding more harvesting pads or speed bumps. 
A moderately busy off ramp with 150,000 Annual Average Daily Traffic (AADT) which is common for off ramps in Canadian cities [39], would produce 300,000 compressions (front and rear tire each compressing the harvesting units once) of a smooth harvesting pad. The optimal total energy generated in a day by eight harvesting pads can then be calculated as:

$$
\left(\frac{300,000 \frac{\text { compressions }}{\text { day }}}{15,807 \frac{\text { compressions }}{4-5 \mathrm{~V}}}\right) \frac{1}{2}(200 \mathrm{~F})\left((5 \mathrm{~V})^{2}-(4 \mathrm{~V})^{2}\right)=17,081 \frac{\mathrm{J}}{\mathrm{day}} .
$$

The average power output for the harvesting pads then becomes:

$$
\left(17,081 \frac{J}{d a y}\right)\left(\frac{1}{24} \frac{d a y}{h}\right)\left(\frac{1}{60} \frac{h}{\min }\right)\left(\frac{1}{60} \frac{\min }{s}\right)=0.1977 \mathrm{~W}
$$

MicroStrain ${ }^{\circledR}$ produces a product called EH-Link ${ }^{\circledR}[40]$. This is a wireless node, which can connect to a variety of sensors and transmit their measurements in addition to measurements from its on board accelerometer, temperature and humidity sensors. The sensor node is designed to run off of "ambient" energy sources including piezoelectric power sources. Using all internal sensors, as well as all external sensor options and operating at the maximum sample rate of $256 \mathrm{~Hz}$ the EH-Link ${ }^{\circledR}$ will consume $18.5325 \mathrm{~mW}$. Choosing lower sampling rates, and using fewer sensors can drastically reduce the power requirements if they are not needed. Ideally then, 8 harvesting pads could power $\frac{0.1977 \mathrm{~W}}{0.0185325 \mathrm{~W}}=10.67$ units or $\sim 1.333$ sensor nodes at full output per harvesting pad. In reality inefficiencies will reduce this number, however this estimate certainly indicates the feasibility of the project. 


\section{Transducer design}

This section introduces the design and modeling of the transducer to be used. First the mathematics behind piezoceramic modeling are covered so that one has an understanding of what the commercial Finite Element Analysis (FEA) software used is doing when calculating solutions. Analytical equations are then introduced and used for estimation purposes of the amount of power that could be harvested by the harvesting circuitry used, and finally the FEA process is covered in order to justify design decisions made.

\subsection{Transducer designs}

There are a variety of transducer designs, piezoelectric devices that convert mechanical work into electricity [38]. In general, different transducers are designed for different loading and displacement scenarios. The simplest form of transducer, called the multilayer transducer, gets its name from its composition simply being several layers of thin PZT plates. The advantage of the multilayer transducer is that it will produce a lower voltage and a higher current when compared to a solid block of PZT, however it will have a similarly high stiffness which limits its power harvesting applications to those that apply very large forces. A more advanced transducer design for power harvesting applications is the THUNDER (THin layer UNimorph ferroelectric DrivER and sensor) transducer which has been implemented in shoe power harvesters [18, 38]. The THUNDER transducer is composed of a curved sheet of PZT laminated between a thin curved aluminum sheet and a curved stainless steel sheet shown in Figure 3.1. Although the THUNDER exhibits good efficiency and power recovery capabilities, its very low stiffness would be inappropriate for the high stresses expected with the wheel load of a vehicle. 


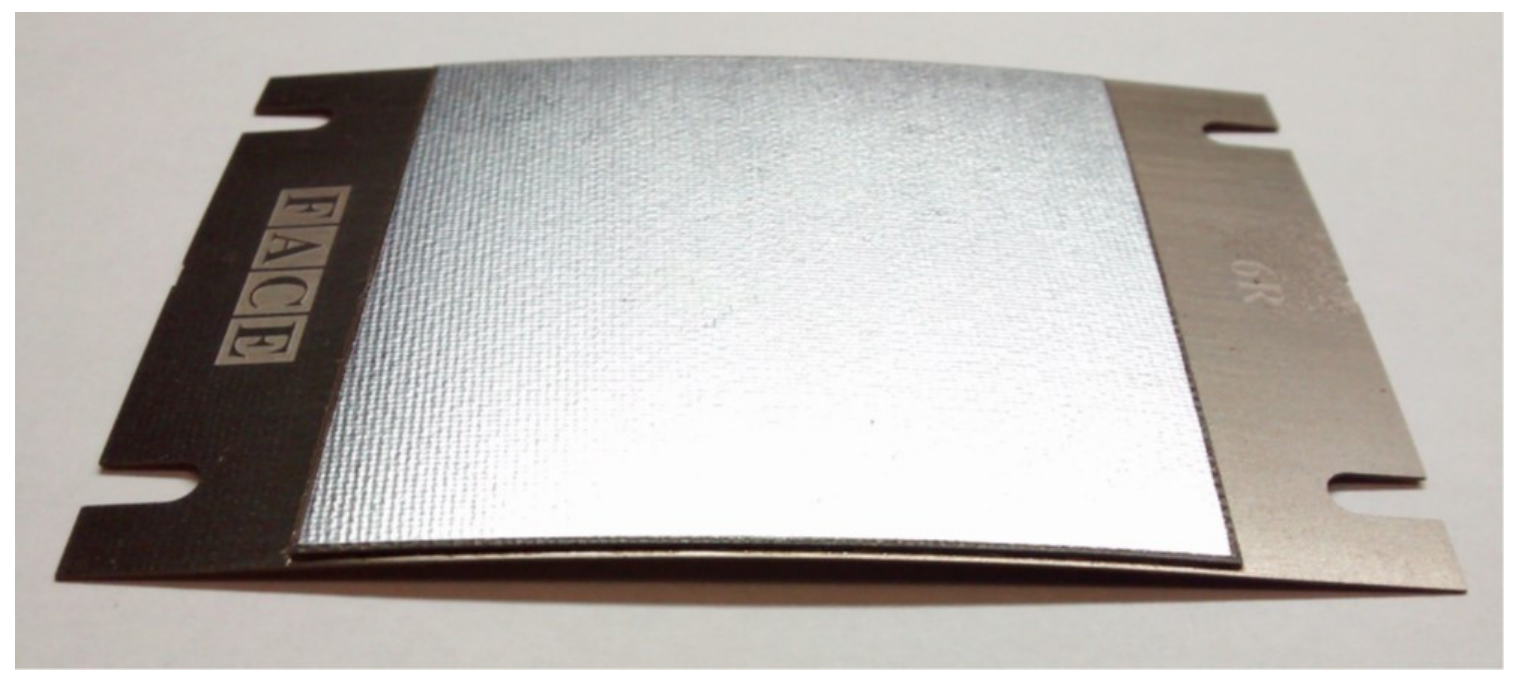

Fig. 3.1: THUNDER transducer manufactured by FACE ${ }^{\circledR}$ International.

The Moonie transducer was developed in 1989 by Xu and Newnham at Pennsylvania State University [41]. Originally designed for use as a hydrophone, transceiver or actuator, the transducer consists of a flat disc of piezoelectric material with two thicker metal discs adhered to either side. Each metal disc has a small hollow pocket adjacent to the piezoelectric material such that they can transform the radial strain of the piezo disc into a linear motion in the dimension normal to the transducer. The Moonie transducer is very flexible in that its metal end caps dimensions can be tailored to suit a variety of applications, however the rigidity of its end caps reduce the transducers effectiveness.

In order to improve on the performance of the Moonie transducer, the manufacturing process can be refined to using a stamped metal disc for the top and bottom end caps. This modified Moonie transducer is referred to as a Cymbal transducer which is shown in Figure 3.2. In the figure, the darker material is the PZT disc, the lighter components are the metal end caps, and the copper coloured material is the bonding epoxy. This transducer is popular because of its design flexibility, similar to the Moonie transducer, but has increased efficiency from the removal of excessive metal which absorbed energy in the form of strain energy [13, 42]. 


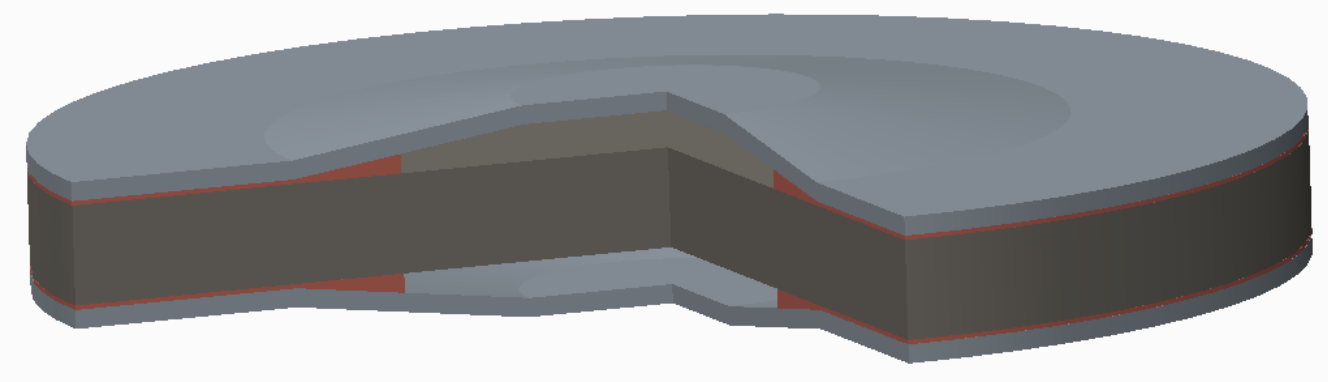

Fig. 3.2: Cymbal transducer with cutout.

Recent research has focused on novel designs aimed at improving performance and/or load tolerance for various or generic applications, some of which are discussed here.

One interesting modification was used to adapt a Cymbal transducer to high depth hydrophone or transmitter by making it more resistant to pressure. This was done by using a piezoelectric disc with a hole in the center and then attaching end caps similar to the Cymbal transducer (Figure 3.2) however inverting the end caps so that two concave cavities are created in the center of the transducer [14]. This is similar in design to [26] which uses a piezoceramic ring to enclose two protruding truncated conical pieces of sheet metal forming a Cymbal inside the ring. The latter transducer design was to achieve a lower profile for power harvesting applications.

Others have been examining the coupling between the end caps and the piezoceramic disc for improving sound generation underwater. Both [13, 15] utilize an outer metal ring which is directly attached to the stamped metal discs, giving more surface attachment area to the piezo unit. This technique helps with transducers which are expected to experience both compressive and tensile radial loads transfer those loads more efficiently to their end caps.

Cymbal transducers generally apply a radial tensile stress to the PZT disc that they typically house. Because of the ceramics poor tensile strength, several researchers have laminated the PZT disc with a metal, typically steel, disc [25, 23]. This would 
have similar drawbacks to MFC's in that the reduced amount of piezoelectric material would also reduce the potential energy available to be harvested in addition to limiting the amount that the piezo material can strain.

Bridge transducers are a simple variant of the Cymbal transducer. Both transducers share exactly the same cross section, but unlike the Cymbals cross section being revolved around the vertical line of symmetry, the Bridge transducer is a linear extrusion of the same cross section. Bridge transducers are useful for applications which require a square or rectangular transducer but are more prone to failure from high loads [38].

The Cymbal transducer was an improvement on the Moonie transducer by removing excess material to reduce the stiffness of the end cap, however further studies have gone on to examining further reductions of material and stiffness of end caps. The study in [24] proposes a circumferential slot be cut into the top of end caps where it begins to bend away from the piezo disc, while the research work in [43] proposes adding radial slits in transducer end caps to reduce circumferential stress and further improve the efficiency of force transmission. While a novel idea, the study in [43] only performed analytical and FEA analysis with the assumption of an infinitely thin radial slit width, a poor assumption that would have been noticed if they had manufactured prototypes. A similar design proposed that much more material may be removed from the end caps in order to produce a "wagon wheel" transducer [44]. In their design, the study in [44] proposes that large triangles be cut out of the end cap material to produce thick spokes radiating from the center of the end caps to the intact edges. This design reduces circumferential stresses, however does so at the expense of removing much of the end cap material, reducing needed end cap integrity for roadway power harvesting.

Larger strains would generate more power, however the drawback to improving the 
mechanical force transmission to the piezoceramics is that the high loading experienced in roadway power harvesting would apply very large tensile forces onto the PZT discs potentially damaging them. Accordingly, we propose combining the concept of a radially slitted transducer to reduce the stiffness of the end cap, with a radially prestressing band encircling the transducers circumference, and applying a compressive load to withstand the high tensile forces of roadway power harvesting. The radial slits have the potential of being more stable than a "wagon wheel" style transducer and the novel prestressing technique would allow it to experience a larger range of strains without failing. While this method of "prestressing" or "preloading" is similar in concept to compressive prestress that is applied to piezoelectric actuators which are expecting large tensile stresses [45], it has never been applied radially to cymbal transducers before. Prestress had been experimented with in the study [46], which applying a compressive constant vertical load. This has the opposite effect which we are striving for however by increasing initial tension within the cymbal transducer. Our novel transducer variant is expected to be able to withstand larger loads while allowing a much larger change in strain from an initially radially compressive state to a radially tensile state when a large load is applied to the transducer. The radial stress is applied by a steel band with a rubber ring buffer. This rubber layer between the transducer and the prestressing band allows the piezo transducer to both expand and contract while approximately maintaining the desired radial prestress. The rubber layer also reduces any stress concentration points that the metal band may impose on the edges of the piezoceramic disc. To further demonstrate the concept, a modified electromechanical model based on one used in [47] will be presented here. The modified model, shown in Figure 3.3, represents a single radially slitted, preloaded transducer. In this model, $F_{l}$ is the variable radial load force applied to the piezoelectric disc by a vehicle through the end cap. This force is applied in an upward fashion, in the model, equating to tension of the PZT component in the model 
as would a cymbal end cap apply a tensile force to a piezoelectric disc. $F_{p}$ is the constant preload force applied by a circumferential steel band which counteracts the load force by applying a radially compressive force to the PZT material. The stiffness $K$ which represents the equivalent combination of the mechanical stiffness's of the end caps and the piezoceramic disc. The equivalent damping is denoted as $C$ and mass $M$. Finally the single dimensional model had direction vector $u$ with a voltage and current associated with the PZT disc being $V$ and $I$.

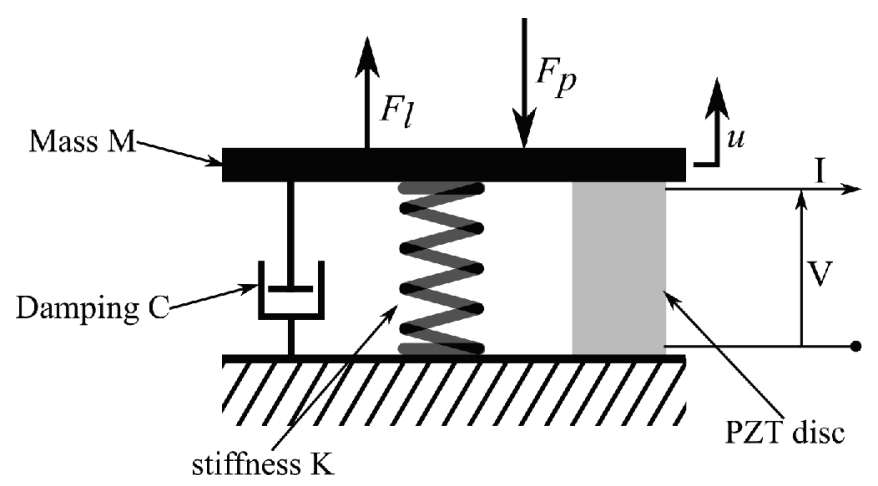

Fig. 3.3: Schematic of a modified electromechanical model for a preloaded Cymbal transducer.

The equation of motion for the model is shown in Equation 3.1.

$$
M \ddot{u}+C \dot{u}+K u+\alpha V=F_{l}-F_{p}
$$

The model can be simplified in order to determine an optimal preload to minimize peak stress in the PZT material. This can be done by assuming the voltage $V$ is zero, and that the vibrations will be slow enough such that the time derivatives of $u$ can be ignored. The new equation becomes:

$$
K u=F_{l}-F_{p}
$$


In order to minimize $\|u\|$ over a range of $F_{l}$ with a preload force $F_{p}, F_{p}$ must be set to approximately half the total expected radial force on the PZT disc, $F_{p}=\frac{F_{l}}{2}$. This is because at rest, $F_{l}=0$, so the PZT disc will be under compressive load $F_{p}$ from the preload band. While under a vehicles load, the piezo disc will experience a radial tension of $F_{l}-F_{p}$. It is assumed that setting $F_{p}=F_{l}$ will give a value close to the minimum in terms of absolute displacement $\|u\|$ and correspondingly close to a minimum stress during the fully loaded condition.

\subsection{Finite element analysis model}

After constructing several preliminary transducers and measuring the parameters after disassembly of the end caps, a model was constructed using the average values found from careful measurements of the preliminary transducers. These values are shown in Table 3.1 and are used to create the 2D axisymmetric model shown in Figure 3.4. Done in COMSOL Multiphysics ${ }^{\circledR}$, and shown in Figure 3.4, the model is axisym-

metric and the upper half of a single, cymbal transducer. COMSOL Multiphysics ${ }^{\circledR}$ was used as an FEA software package for its ability to model piezoelectric materials and the internal electric fiends that change the materials mechanical properties. 


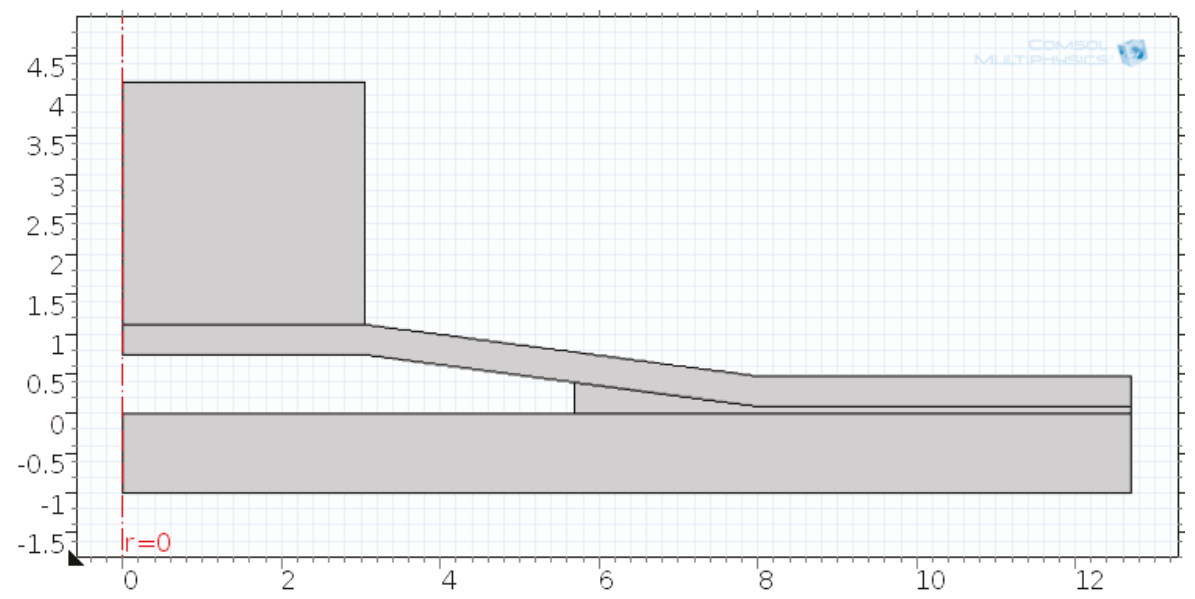

Fig. 3.4: Basic axisymmetric model geometry.

The geometry shown in Figure 3.4 is an axisymmetric half model in order to decrease computation time while performing large parameter sweeps to explore the parameter or design space. Early FEA models applied a uniformly distributed force to the top of the end cap geometry shown in Figure 3.4. This method for applying forces would cause the flat surface of the top of the end cap to bow downward generating high and unrealistic stress concentrations. In order to apply the force more realistically to the top of the end cap of the FEA model, a puck shaped block was placed and is seen as a square on top of the geometry. This is a nylon block, used to apply pressure to the top of the end cap in the same way that pressure is intended to be applied though the cast nylon casing that will house the transducers. The values of the parameters governing the model are shown in Table 3.1 and the parametric model in Figure 3.5. The model geometry shown in Figure 3.4 appears to be one quarter of the parametric model, depicted as a full cross section in Figure 3.5, however since the model is axisymmetric, the geometry is revolved around the red axis on the left side of Figure 3.4, the basic geometry actually represents the upper half of a cymbal transducer. 
Table 3.1: Model default parameters

\begin{tabular}{|c|c|l|}
\hline Variable name & Value & \multicolumn{1}{|c|}{ Description } \\
\hline \hline t_endCap & $0.38[\mathrm{~mm}]$ & Thickness of the end cap. \\
\hline h_endCap & $0.65[\mathrm{~mm}]$ & Height of cavity. \\
\hline d_epoxy & $7.01[\mathrm{~mm}]$ & Epoxy ooze depth from the outside radius. \\
\hline r1 & $3.05[\mathrm{~mm}]$ & $\begin{array}{l}\text { Radius of the smallest bend at the top of the end } \\
\text { cap. }\end{array}$ \\
\hline r2 & $8.00[\mathrm{~mm}]$ & $\begin{array}{l}\text { Radius of the bend where the end cap and PZT } \\
\text { are bonded. }\end{array}$ \\
\hline t_epoxy & $0.088[\mathrm{~mm}]$ & The thickness of the epoxy. \\
\hline r_edge & $12.7[\mathrm{~mm}]$ & Radius of the and cap itself. \\
\hline Fapp & $367.875[\mathrm{~N}]$ & The applied force. \\
\hline t_PZT & $2[\mathrm{~mm}]$ & The thickness of the PZT disc. \\
\hline
\end{tabular}

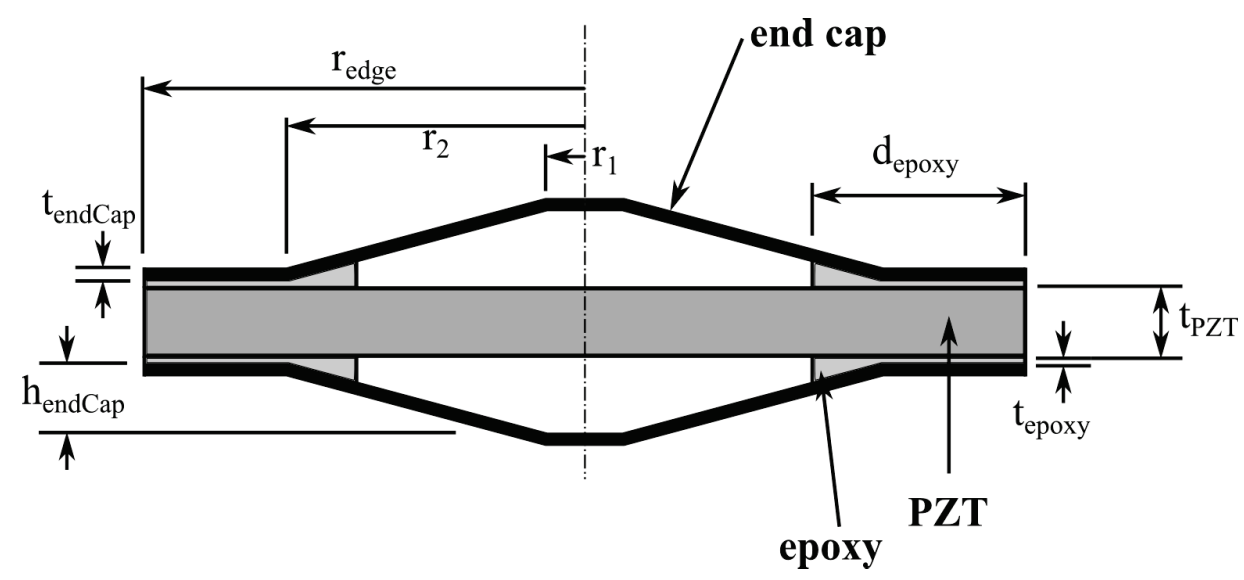

Fig. 3.5: Parametric cross section model of a Cymbal transducer for FEA purposes.

The force applied to the cymbal transducer model was calculated assuming a vehicle weight of $1,500 \mathrm{~kg}$ and 10 power harvesting transducers in one cast nylon transducer casing. It is assumed that the block of ten transducers will be supporting the entire weight on a single tire and that each tire supports one quarter of the vehicle weight. The calculation is shown in Equation 3.3:

$$
F_{\text {app }}=1,500 \mathrm{~kg} \cdot 9.81 \frac{\mathrm{N}}{\mathrm{kg}} \cdot \frac{1}{4} \text { tires } \cdot \frac{1}{10} \text { transducers. }
$$


In order to perform FEA on a geometry, the assembly must be reduced to a series of finite elements. The density of these elements roughly correlates to the intricacy of the analysis that the model is capable of. Unless otherwise stated, the model was meshed such that the features of the model, especially small ones such as the epoxy layer and epoxy ooze shown in Figure 3.5, were spanned by several finite elements. This was done to ensure accuracy at the expense of computation time. The basic mesh assigned to the default geometry is shown in Figure 3.6.

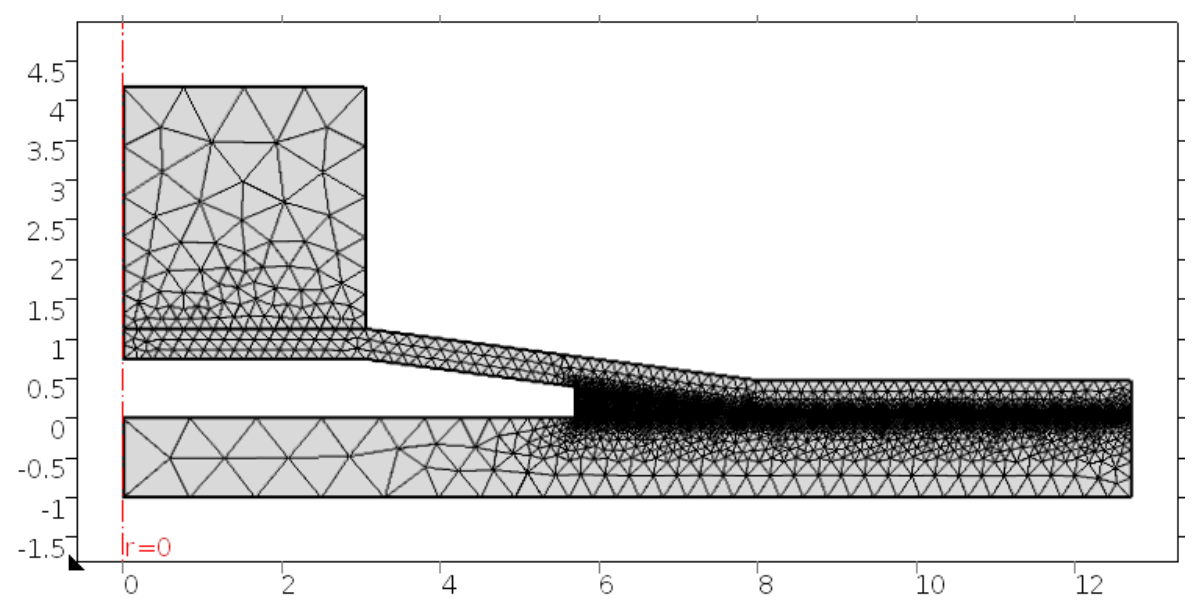

Fig. 3.6: FEA geometry mesh.

Governing equations for the FEA model were defined in Chapter 1: Introduction as Equations Piezoelectric notation and Piezoelectric notation but are repeated here for clarity.

$$
\begin{gathered}
x=c^{E} X-e_{E S} E \\
D=e_{E S} X+\varepsilon E
\end{gathered}
$$


where $c^{E}, e_{E S}$, and $\varepsilon$ are material properties defined in Section 3.2.1. $E$ is the applied electric field, $X$ is the strain, $x$ is the internal stress, and $D$ is the surface charge density. These equations apply to the piezoelectric material component of the model only, shown in Figure 3.5. The rest of the material follows the governing equations derived from Hooke's law:

$$
x=Y_{x y} X
$$

where $Y_{x y}$ is the materials Young's modulus and can be related to the elastic compliance with Equation 1.18.

All axisymmetric models applied the symmetry axis about the red line in Figure 3.6 and bounded the bottom edge of the model to only displace radially (a symmetric constraint). The load, shown in Table 3.1, is applied to the model as an evenly distributed load to the top edge of the model.

\subsubsection{Material selection}

The materials used in the model along with their properties are shown in Table 3.2. 
Table 3.2: Materials used and their properties.

\begin{tabular}{|c|c|c|}
\hline Material & Properties & Purpose \\
\hline Cast nylon [48] & $\begin{array}{c}\text { Density: } 1,150 \frac{\mathrm{kg}}{\mathrm{m}^{3}} \\
\text { Young's modulus: } 2 \times 10^{9} \mathrm{~Pa} \\
\text { Poisson's ratio: } 0.4 \\
\text { Yield strength: } \\
(6.89-9.31) \times 10^{7} \mathrm{~Pa}\end{array}$ & $\begin{array}{l}\text { To emulate a machined } \\
\text { nylon harvesting } \\
\text { container and to reduce } \\
\text { stress concentrations } \\
\text { around end cap contact } \\
\text { points. }\end{array}$ \\
\hline $\begin{array}{l}\text { 301 Stainless } \\
\text { steel [48] }\end{array}$ & $\begin{array}{c}\text { Density: } 7,880 \frac{\mathrm{kg}}{\mathrm{m}^{3}} \\
\text { Young's modulus: } 1.93 \times 10^{11} \mathrm{~Pa} \\
\text { Poisson's ratio: } 0.33 \\
\text { Yield strength: } 9.65 \times 10^{8} \mathrm{~Pa}\end{array}$ & $\begin{array}{c}\text { A high stiffness and } \\
\text { high strength end cap } \\
\text { material. }\end{array}$ \\
\hline 510 Bronze [48] & $\begin{array}{c}\text { Density: } 8,857 \frac{\mathrm{kg}}{\mathrm{m}^{3}} \\
\text { Young's modulus: } 1.103 \times 10^{11} \mathrm{~Pa} \\
\text { Poisson's ratio: } 0.4 \\
\text { Yield strength: } 5.51 \times 10^{8} \mathrm{~Pa}\end{array}$ & $\begin{array}{l}\text { Another widely used } \\
\text { end cap material. }\end{array}$ \\
\hline $\begin{array}{l}\text { Lepage }{ }^{\circledR} \\
\text { Professional } \\
\text { Speed } \\
\text { Set }^{\mathrm{TM}} \text { Epoxy }\end{array}$ & $\begin{array}{l}\text { Density: } 1,150 \frac{\mathrm{kg}}{\mathrm{m}^{3}} \\
\text { Young's modulus: } 2 \times 10^{9} \mathrm{~Pa} \\
\text { Poisson's ratio: } 0.4\end{array}$ & $\begin{array}{c}\text { The epoxy to be used to } \\
\text { bond the end cap to the } \\
\text { PZT disc. }\end{array}$ \\
\hline APC 855 [28] & $\begin{array}{c}\text { Density: } 7,600 \frac{\mathrm{kg}}{\mathrm{m}^{3}} \\
\text { Elasticity matrix: } c_{E} \\
\text { Coupling matrix: } e_{E S} \\
\text { Relative permittivity: } \varepsilon \\
\text { Yield strength: } 2.76 \times 10^{7} \mathrm{~Pa} \\
(4000 \mathrm{psi})\end{array}$ & $\begin{array}{l}\text { The Piezoelectric } \\
\text { material to be used. }\end{array}$ \\
\hline Rubber & $\begin{array}{l}\text { Density: } 1,100 \frac{\mathrm{kg}}{\mathrm{m}^{3}} \\
\text { Young's modulus: } 3.8 \times 10^{8} \mathrm{~Pa} \\
\text { Poisson's ratio: } 0.55\end{array}$ & $\begin{array}{l}\text { To behave as a buffer } \\
\text { between the preloading } \\
\text { band and the piezo } \\
\text { transducer. }\end{array}$ \\
\hline
\end{tabular}


where:

$c_{E}=\left[\begin{array}{cccccc}127.205 & 80.2122 & 84.6702 & 0 & 0 & 0 \\ & 127.205 & 84.6702 & 0 & 0 & 0 \\ & & 117.436 & 0 & 0 & 0 \\ & & & 22.9885 & 0 & 0 \\ & & & & 22.9885 & 0 \\ & & & & & 23.4742\end{array}\right] \mathrm{GPa}$

and

$$
e_{E S}=\left[\begin{array}{ccc}
0 & 0 & -111.11 \\
0 & 0 & -111.11 \\
0 & 0 & 47.619 \\
0 & 37.037 & 0 \\
37.037 & 0 & 0 \\
0 & 0 & 0
\end{array}\right] \frac{C}{m^{2}}
$$

finally

$$
\varepsilon=\left[\begin{array}{ccc}
1704.4 & 0 & 0 \\
0 & 1704.4 & 0 \\
0 & 0 & 1433.6
\end{array}\right] \frac{F}{m} .
$$

As previously mentioned, examining the results of the FEA model shown in Figure 3.6, it was noticed that adding a nylon cylinder push block to the top of the transducer was needed to avoid unrealistic stress concentrations in the load bearing component of the end cap. The end caps themselves are made of 301 stainless steel by default 
in the models because the material is very strong, and can withstand smaller end cap thicknesses. The 510 bronze, also known as phosphor bronze, was used in other transducer designs $[24,38,41,49]$, and in our initial prototypes. The Lepage ${ }^{\circledR}$ Professional Speed Set ${ }^{T M}$ Epoxy proved to be a capable adhesive in early prototypes, and so was used in the modeling. APC 855 was used due to reasons described in Section 2.2 and finally, the rubber was used as a buffer, reducing stress concentrations, and allowing the transducer to expand in the model.

\subsubsection{Endcap thickness model}

In order to determine the effect of end cap thickness on the output power produced, the model was set up to step through a range of possible end cap thicknesses using 301 stainless steel end caps. This is the material that will later be chosen for use on the final prototypes due to its high stiffness and yield strength. The thickness ranged from $0.0254 \mathrm{~mm}$ to $0.381 \mathrm{~mm}$ with a step size of $0.0254 \mathrm{~m}$. These correspond to a step size and initial thickness of $0.001 "(0.0254 \mathrm{~mm})$ and a final thickness of 0.015 " $(0.381 \mathrm{~mm})$ as the thicknesses available for purchase tend to be in imperial units and thus in thousandths of an inch [48]. The maximum stress in the PZT material, the end cap material, and total electric energy from the model was recorded and is plotted against end cap thickness in Figures 3.7, 3.8, and 3.9, respectively. 


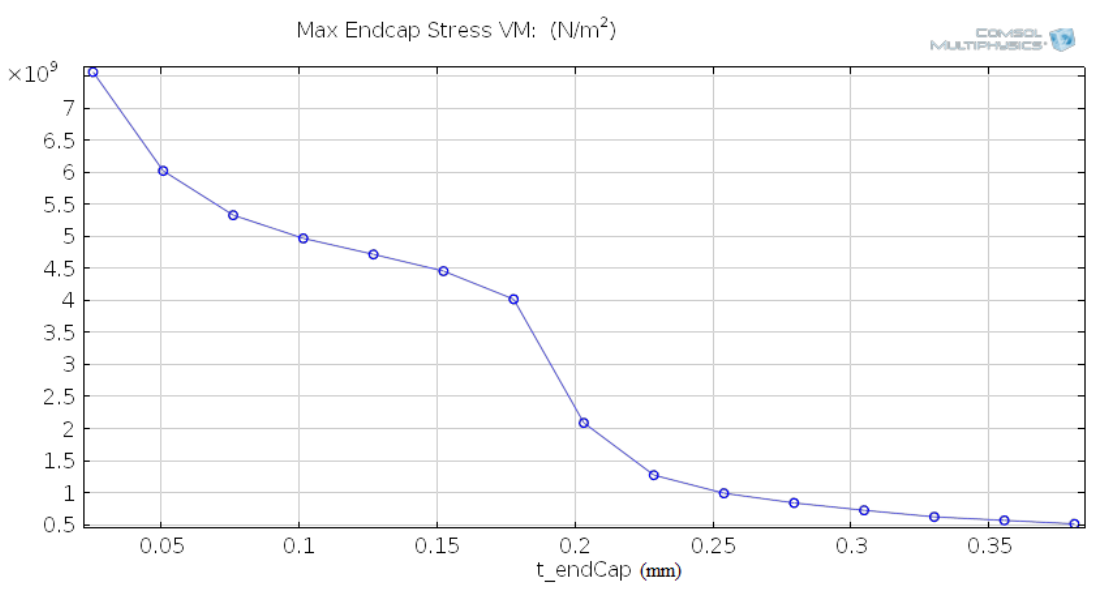

Fig. 3.7: Maximum calculated stress in the stainless steel end cap.

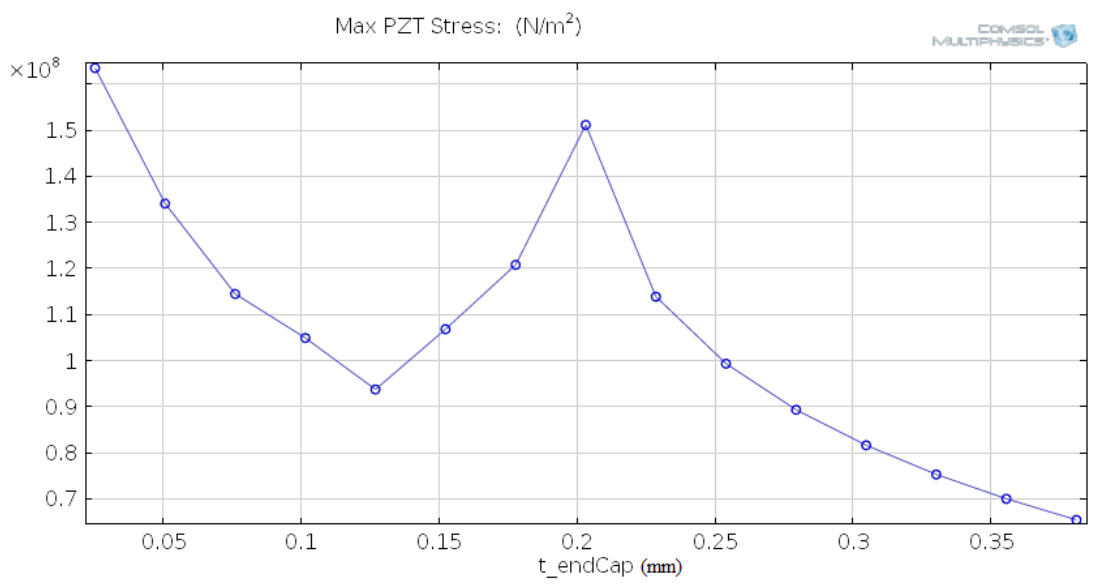

Fig. 3.8: Maximum calculated stress in the piezo disc.

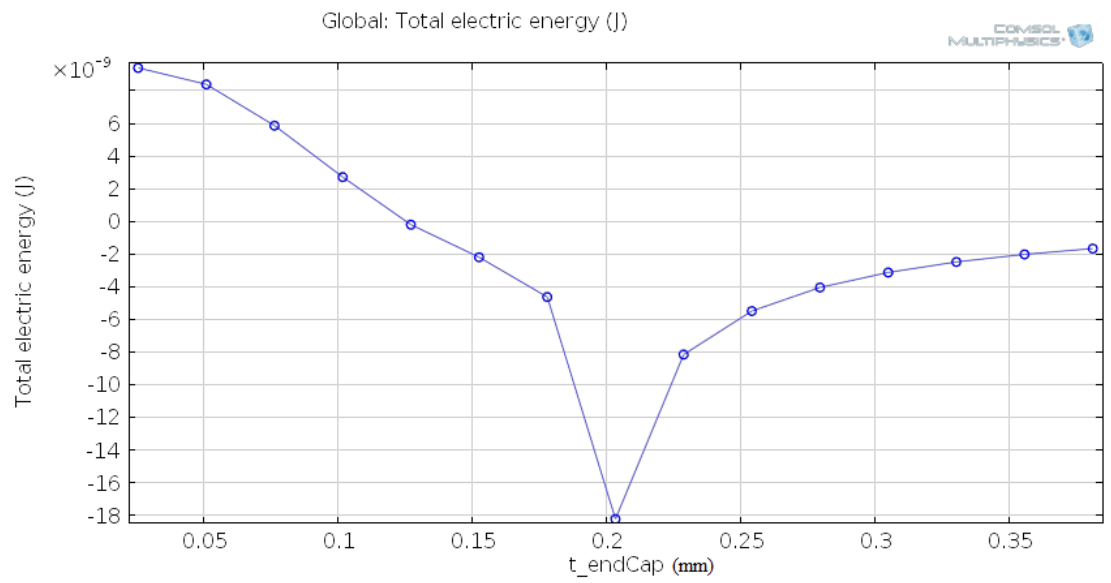

Fig. 3.9: Total electric energy produced under compression. 
From these models, it can be seen that a solid end cap thickness of about 0.008 " $(0.2032 \mathrm{~mm})$ has the highest potential to produce the most electricity however it results in the maximum stress of the PZT material that exceeds its yield strength. This result is later tested with fabricated prototypes. It is noted that the range of peak stresses shown in Figure 3.8 are all above the failure strength which was reported by the manufacturer, APC, which reported it as being between 2,000 and 4,000 psi $(13.8 \mathrm{MPa}-27.6 \mathrm{MPa})$ in tension and 6,000 and 8,000 psi (41.4 MPa-55.2 MPa) in compression. Transducers prototyped, built, and tested in Chapter 4 have performed well without damage to the PZT disc, indicating that the reported failure values may have a safety factor associated with them. Although preloading methods will be explored later in this paper to reduce the peak stresses below the maximums suggested by APC, the largest end cap thickness of $0.015 "(0.381 \mathrm{~mm})$ is chosen as a conservative parameter value.

\subsubsection{Epoxy ooze model}

It was noticed when deconstructing old cymbal transducer prototypes that often a large amount of epoxy had oozed into the central cavity. It was decided to simulate this phenomenon in order to see if it has a significant effect on the cymbals power production and internal stresses. The model varied the depth of epoxy ooze from having no ooze (just epoxy where the end cap would contact the piezoelectric material) to the point where the epoxy filled into the cavity up to the smaller radius bend, truncating the cymbals cone. This was simulated assuming the default model thickness for the end cap.

The stresses in the end cap and PZT are shown in Figures 3.10, and 3.11. More importantly however, the total power generated is shown in Figure 3.12 which shows that there is an inverse relationship between epoxy ooze and electrical energy gener- 
ated. This indicated that transducers must be built consistently with as little epoxy ooze as possible.

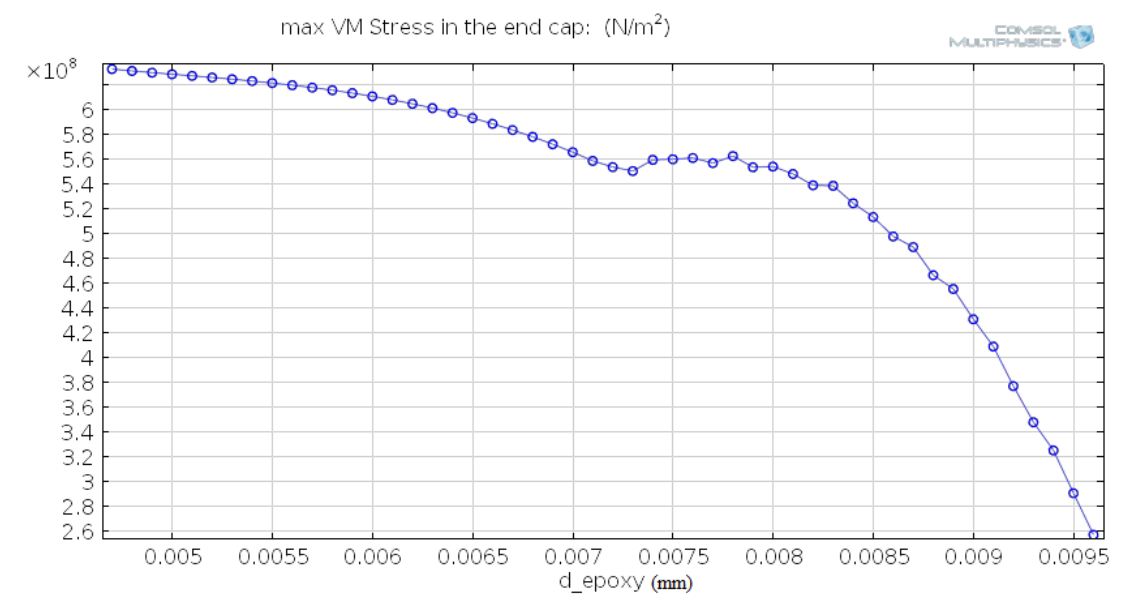

Fig. 3.10: Maximum stress in the end cap while varying the ooze depth.

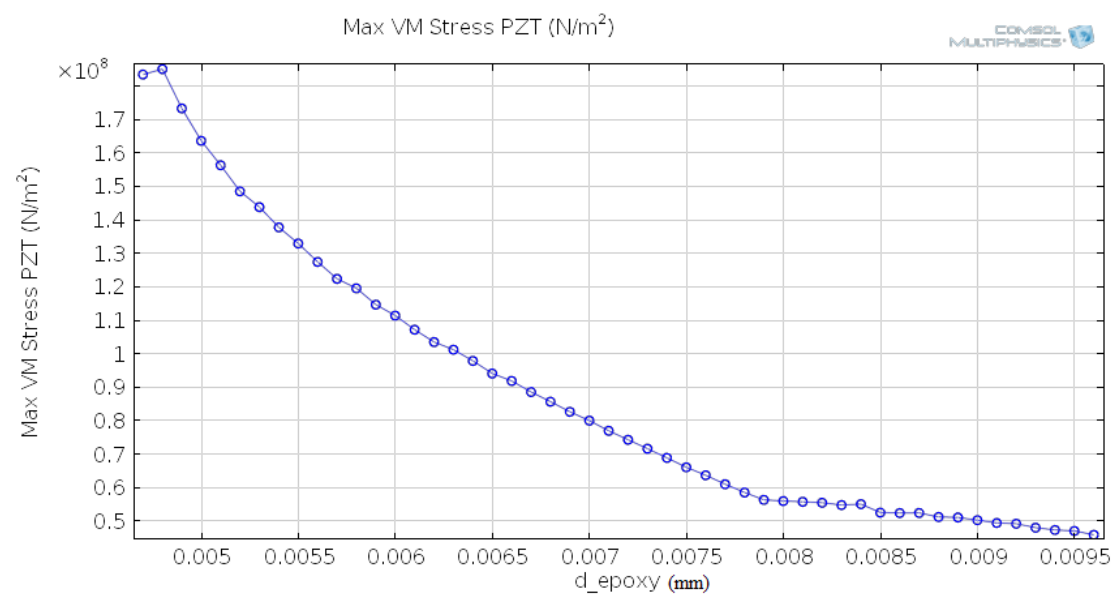

Fig. 3.11: Max stress in the piezo material while varying ooze depth. 


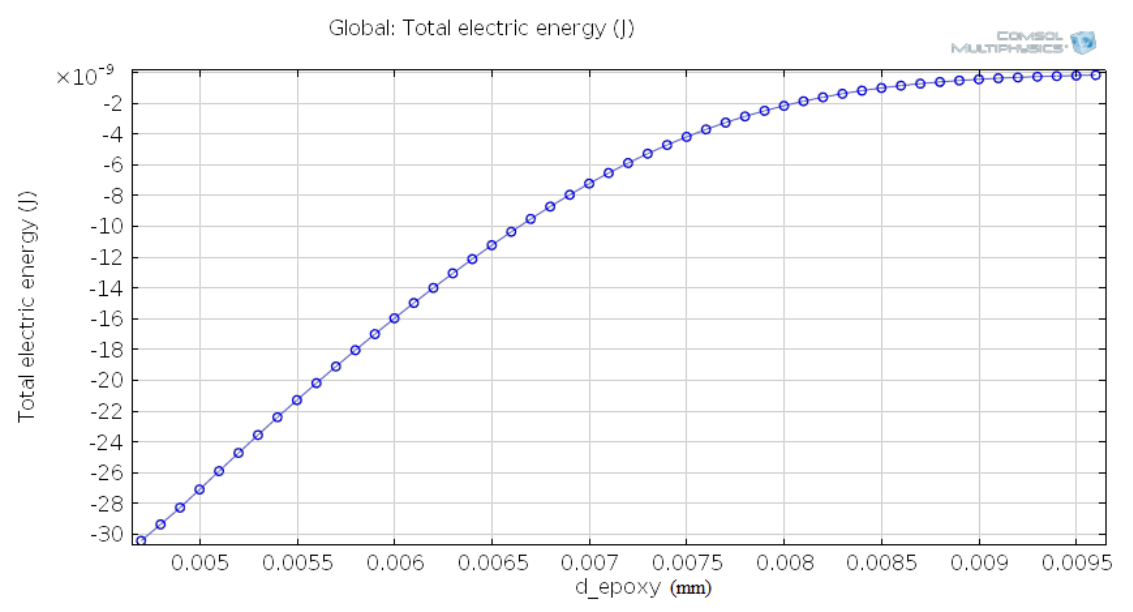

Fig. 3.12: Electrical energy generated as a function of ooze depth.

The simulation was repeated with smaller end cap thicknesses, which resulted in similar shaped electrical energy plots as shown in Figure 3.12. This indicates that the epoxy ooze depth into the central cavity should be kept to a minimum to maintain transducer performance. This analysis caused prototypes to be manufactured with care to use as little epoxy as needed to reduce epoxy ooze and degraded transducer performance as much as possible.

\subsubsection{FEA preload model}

Qualitatively, the preload model initially showed little improvement in reducing the peak stress in the PZT material. Upon further inspection, it appeared that the simulated metal band (which was placed adjacent to the PZT disc) was causing stress concentrations to form between it and the PZT disc and negating its peak stress reduction. A preload band directly contacting a transducer could cause a short circuit, reducing charge generation. A buffer band was added to the model with an estimated Young's modulus, calculated from rubber stretch tests described later in this section. This modification led to a large improvement in reduction of stress on the PZT disc. As the Young's modulus of the band material was decreased (simulating a 
softer, more pliable material), the ideal preload stress was found to increase. It has made the study of preloading difficult as the ideal amount of preload stress seems highly dependent on how hard the buffering material is between the preload band and the PZT disc.

The simulation indicates that the buffer banding should be a very pliable material such as rubber, and that the preload stress can be quite high with such a pliable material. The preload model used an updated set of default model parameters shown in Table 3.3 for the parametric model shown in Figure 3.5 which are the result of more careful measurements being taken of disassembled transducers. In addition, the preload model incorporated a preload band and buffering rubber band. The preload band was chosen to be $0.5 \mathrm{~mm}$ thick however any thickness would have been sufficient as the important information desired from the model is optimal preload stress. The rubber buffer layer's thickness was obtained from measurements of the diameter of the cymbal transducer with three rubber bands around it. The rubber bands chosen were originally designed for holding lobster claws closed. This type of band was chosen because of their high thickness, ample height large enough to accommodate the thickness of one or two transducers, and circular diameter smaller than one inch so that they would encircle a 1" PZT transducer without slack. Three bands were chosen due to a compromise between the transducer size and the improved performance of having more buffing material. The updated parameters, however, did not incorporate a smaller epoxy ooze depth measurement, d_epoxy, due to the instability it caused in the nonlinear model. 
Table 3.3: Model updated default parameters.

\begin{tabular}{|l|l|l|}
\hline Variable Name & Value & Description \\
\hline \hline t_endCap & $0.38[\mathrm{~mm}]$ & Thickness of the end cap \\
\hline h_endCap & $0.62[\mathrm{~mm}]$ & Height of cavity \\
\hline d_epoxy & $7.01[\mathrm{~mm}]$ & Epoxy ooze depth from the outside radius \\
\hline r1 & $3.34[\mathrm{~mm}]$ & $\begin{array}{l}\text { Radius of the smallest bend at the top of the end } \\
\text { cap }\end{array}$ \\
\hline r2 & $7.97[\mathrm{~mm}]$ & $\begin{array}{l}\text { Radius of the bend where the end cap and PZT } \\
\text { are bonded }\end{array}$ \\
\hline t_epoxy & $0.079[\mathrm{~mm}]$ & The thickness of the epoxy \\
\hline r_edge & $12.7[\mathrm{~mm}]$ & Radius of the and cap itself \\
\hline Fapp & $367.875[\mathrm{~N}]$ & The applied force \\
\hline t_PZT & $1[\mathrm{~mm}]$ & The thickness of half of the PZT disc \\
\hline t_rubb & $2.45[\mathrm{~mm}]$ & Thickness of the preload rubber \\
\hline t_band & $0.5[\mathrm{~mm}]$ & Thickness of the end cap preload band \\
\hline
\end{tabular}

In order to derive an estimate for the elastic modulus of the rubber, the elastics were warped around two small metal bars, one was fixed to a table, the other for attaching weights to. The rubber elastics were measured to be $27.96 \mathrm{~mm}$ long (flattened with no load), $24.60 \mathrm{~mm}$ with no weight on the test rig, $40.90 \mathrm{~mm}$ with a single kilogram mass, and $64.62 \mathrm{~mm}$ with a two kilogram mass. The elastic is measured to be $1.55 \mathrm{~mm}$ thick and $12.28 \mathrm{~mm}$ wide. The cross sectional area is then calculated as $1.55 \mathrm{~mm}$. $\frac{1 \mathrm{~m}}{1,000 \mathrm{~mm}} \cdot 12.28 \mathrm{~mm} \cdot \frac{1 \mathrm{~m}}{1,000 \mathrm{~mm}}=1.9034 \times 10^{-9} \mathrm{~m}^{2}$. The Young's modulus is defined as $E=\frac{F / A_{0}}{\Delta L / L_{0}}[32]$ so using the stretched measurements gathered, the elastic modulus becomes:

$$
\begin{gathered}
E_{1}=\frac{9.81 /\left(2 \cdot 1.9034 \times 10^{-9}\right)}{((40.9-24.6) / 24.6) / 1000}=3.8892 \times 10^{8} \mathrm{~Pa} \\
E_{2}=\frac{(2 \cdot 9.81) /\left(2 \cdot 1.9034 \times 10^{-9}\right)}{((64.62-24.6) / 24.6) / 1000}=3.1681 \times 10^{8} \mathrm{~Pa}
\end{gathered}
$$

The preloaded transducer was modeled using structural nonlinearities since the geometry changed significantly due to the flexing of the end cap and compression of the 
preload band. As such, the model was solved iteratively by COMSOL Multiphysics ${ }^{\circledR}$ until the model deformation was estimated correctly. A simplified mesh was used as shown in Figure 3.13 in order to improve the model stability, and improve the overall solution times for the large sets of parametric studies performed. The labeled elements in the figure from right to left are the preload band, followed by a rubber buffer layer and finally the transducer.

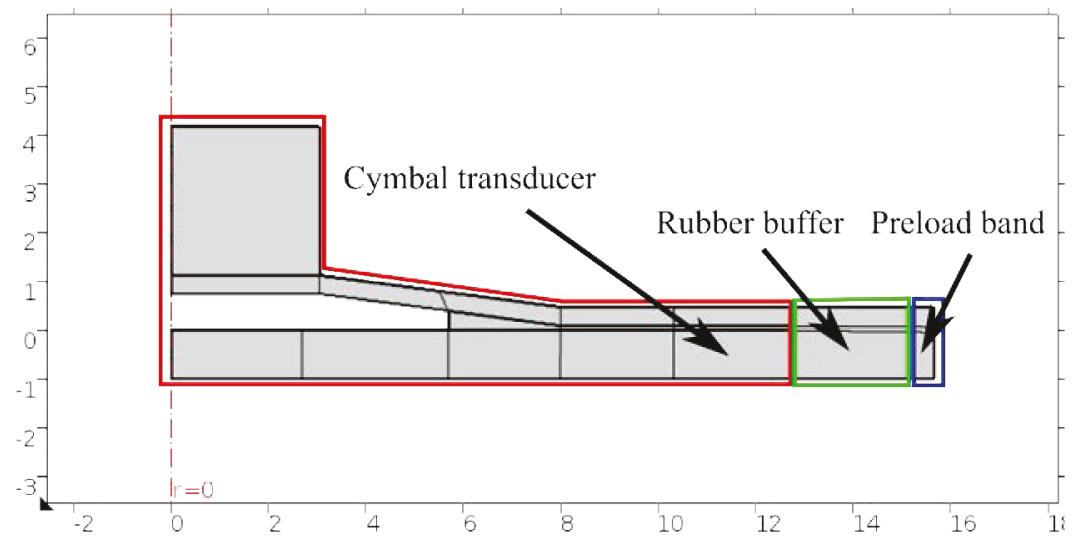

Fig. 3.13: Preload model FEA mesh.

The preload was applied to the model by modifying the initial stress within the preload band region shown in Figure 3.13. The initial stress was set to:

$$
x_{0}=\left[\begin{array}{ccc}
0 & 0 & 0 \\
0 & 0 & 0 \\
0 & 0 & -x_{p r l d}
\end{array}\right]
$$

where $x_{p r l d}$ is the initial preload stress within the preload band.

The model underwent a series of studies in which the end cap thickness and the preload tension were varied in order to find the optimum preload stress through a variety of end cap thicknesses. Early hydraulic testing experiments demonstrated that the epoxy layer and the PZT disc were the most susceptible to failure and so 
examination of the peak stresses in both the epoxy and the piezo material are critical to increasing transducer performance and damage resistance.

The FEA studies varied the initial preload stress, $x_{\text {prld }}$, from $0 \mathrm{GPa}$ to $8 \mathrm{GPa}$ which manifests as increasing preloading tension in the band and compression of the Cymbal transducer. The end cap thickness was also varied from $0.127 \mathrm{~mm}$ to $0.381 \mathrm{~mm}$ in $0.0127 \mathrm{~mm}$ increments. This is the equivalent of 0.005 " to 0.015 " inches thick, the range of stainless steels being available considered for use in preloaded transducers. Figure 3.14 shows the peak stress within the piezo disc region of the FEA model while at full vehicle wheel load calculated in Equation 3.3 and shown in Table 3.1 as "Fapp". This demonstrates that an optimum preload tension exists for a given end cap thickness that minimizes peak stresses in the piezo material. The preloading tension is calculated and included in Figure 3.14 by calculating the tension given the initial preload stress, $x_{p r l d}$, and the cross sectional area of the preload band given by the parameters t_band, t_PZT, t_epoxy, and t_endCap. Optimal preload stress appears linearly dependent on a given end cap thickness. Using the optimal preload tensions for each end cap thickness, a linear regression was performed to relate end cap thickness to optimal full load preload tension and is shown in Equation 3.8 with an $R^{2}=0.9943$

$$
F_{p}=-16,367.64 \cdot \mathrm{t} \_ \text {endCap }+11,205.90 \mathrm{~N}
$$

This does not correspond to a minimization of stress through a load cycle however. It is assumed that to minimize the stress in the PZT element for an entire load cycle, the optimum preload stress would apply half the compressive load of the full load optimal preload tension. This is to prevent excessive compressive loading on the transducer 
while it is unloaded.

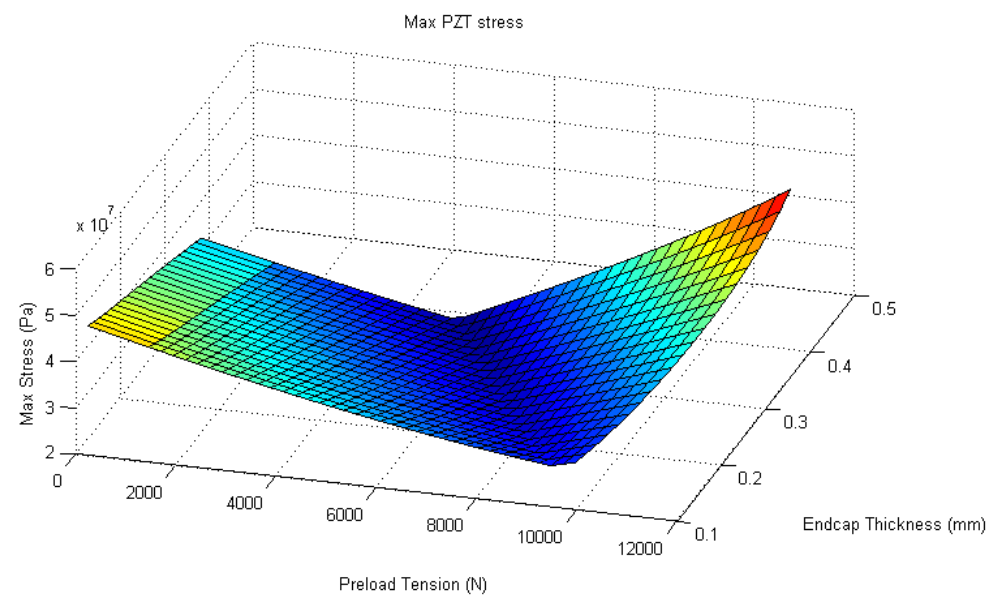

Fig. 3.14: Peak piezo material stress for the range of end cap thicknesses and preload tensions examined.

Because of the susceptibility of the epoxy to fail, consideration was also given to examining the peak stress in the epoxy region under the same range of preloading and end cap thicknesses. Figure 3.15 shows those peak stresses, which indicates that for the range of preload and end cap thickness cases solved, the stress within the epoxy bond is reduced with increased preload tension and increased end cap thickness.

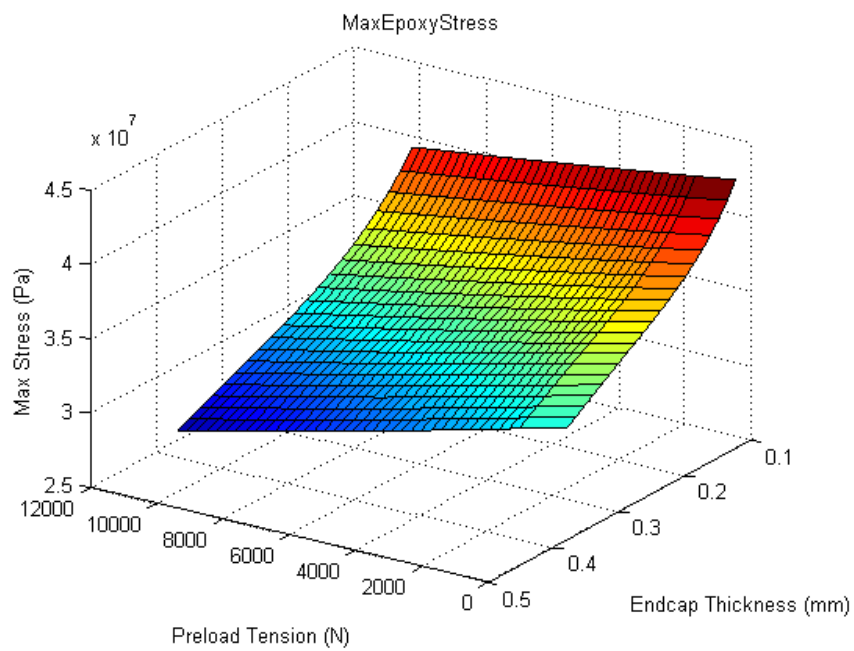

Fig. 3.15: Peak stress in the epoxy region of the model in a range of and cap thicknesses and preload tensions tested.

Finally the effect of preloading and end cap thickness on the end caps peak stress was 
examined and the results are shown in Figure 3.16. Although the preload seems to have little effect on the peak end cap stress, it does has the effect of reducing the peak stress experienced by the end cap. The largest determiner of stress on end caps is the end cap thickness with smaller end cap thicknesses drastically increasing internal end cap stresses, preload tension, and very slightly reducing internal stresses. Referencing Table 3.2 the thinnest safe end cap thickness would be $0.2032 \mathrm{~mm}$ or 0.008 " thick. These results indicate that preloading can be used to reduce the peak piezoelectric and end cap materials stresses and is explored with prototypes later in this thesis.

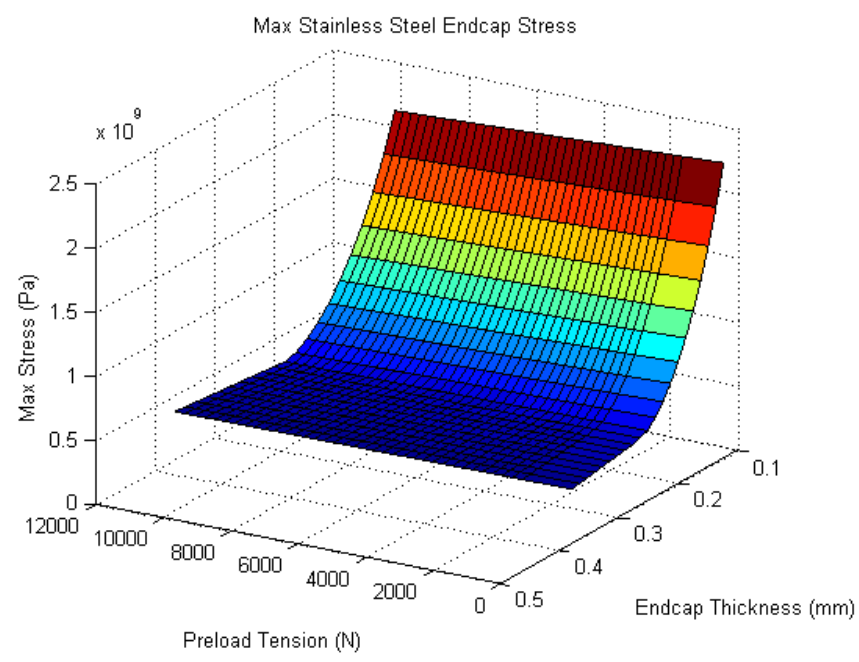

Fig. 3.16: Peak stress in the stainless steel end cap region of the model in a range of and cap thicknesses and preload tensions.

\subsubsection{Analytical preload model}

Due to the very large finite elements in the FEA model, an analytic model as created to verify the FEA results. In order to place an upper bound the preload tension needed, a model is devised where the end cap is divided into $N$ thin wedges where cylindrical stresses are ignored. The introduction of cylindrical stresses to the model would reduce the amount of preloading required due to the end caps applying a circumferential tension, supplementing the preloading tension. One of these extremely 
thin wedges is shown in Figure 3.17. The radially compressive stress that would cause the radial stress in the PZT disc to be zero for a single wedge would then become:

$$
F_{r}=\frac{r_{2}-r_{1}}{h_{\text {endCap }}}\left(\frac{F_{a p p}}{N}\right) .
$$

where $F_{r}$ is the total radial compressive force on a single wedge.

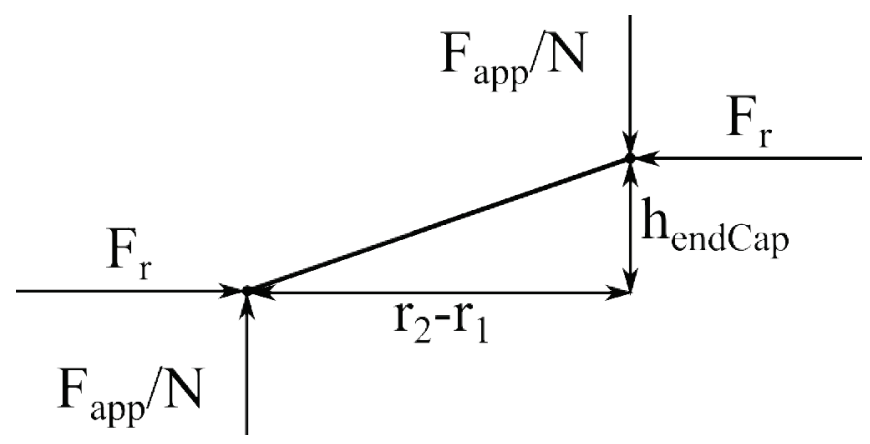

Fig. 3.17: Analytic preload model cross section.

Figure 3.18 shows a top view of a wedge of the model. Ignoring the cylindrical forces causes the model to estimate an upper bound for preload forces. Radially slitted end caps are designed to experience smaller cylindrical stresses and an underestimation of preload tension may make a preloaded transducer more susceptible to damage so the overestimate which model will make is not undesirable. Using Figure 3.18, the radial tension, $F_{t}$, needed to compensate for a radial force $F_{r}$ would become:

$$
\begin{gathered}
F_{t}=\frac{F_{r}}{2 \sin \left(\frac{\pi}{N}\right)} \\
F_{t}=\frac{\frac{r_{2}-r_{1}}{h_{\text {endCap }}}\left(\frac{F_{a p p}}{N}\right)}{2 \sin \left(\frac{\pi}{N}\right)} .
\end{gathered}
$$




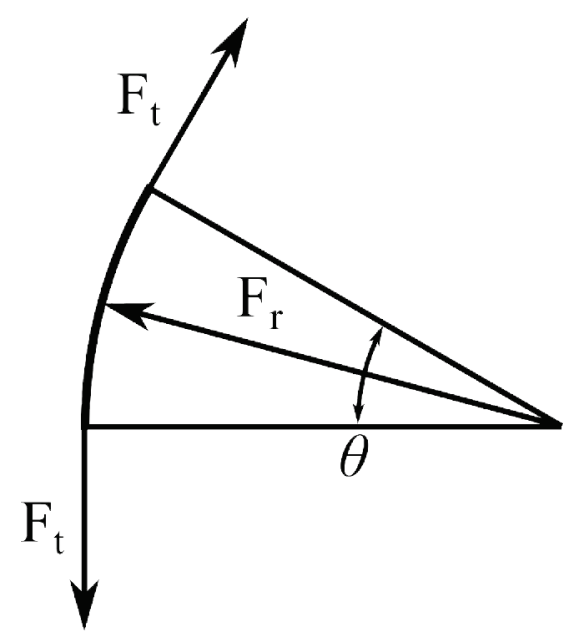

Fig. 3.18: Analytic model top view.

If we assume the wedge angle, $\theta$, is infinitely small, then that implies that we will have an infinite number of wedges. Letting $N \rightarrow \infty, F_{t}$ then becomes:

$$
\lim _{N \rightarrow \infty} F_{t}=\frac{\left(r_{2}-r_{1}\right) F_{a p p}}{2 \pi h_{\text {endCap }}} .
$$

This estimate shown in Equation 3.11 will over estimate the preload stress, because the stiffness and thickness of the cymbal end cap is not taken into account, which would reduce the need to apply additional compressive force. Inputting the cymbal parameters from Table 3.3 into Equation 3.11 produces an estimate for preload tension of $438.41 \mathrm{~N}$. This value is later used to calculate the tension to be set in the prototype transducers. Similar to the results shown in Figure 3.14 and Equation 3.8, this amount of preload tension represents the value which would minimize the internal radial stress of the PZT disc at maximum loading, and not minimizing the stress for the range of loading the transducer is expected to experience. The analytical upper bound 
for preload band tension, calculated to be $438.41 \mathrm{~N}$, can be compared to the lowest estimate given by Equation 3.8 which is calculated as $4969.8 \mathrm{~N}$ assuming an end cap thickness of $0.381 \mathrm{~mm}$. The large discrepancy between the analytical solution and the FEA solution may indicate that the mesh density in the preload FEA model is likely too low and the numerical results from that study are unlikely to be accurate.

\subsubsection{Slit model}

The ultimate goal of the FEA slitted model was to determine the effect and appropriate number of radial slits in a cymbal transducer design. The paper in [43] performed analysis of similarly radially slitted cymbal transducers, examining both an analytic model and FEA model. They however made the assumption that the radial slits would be very thin. It was decided that the easiest way to cut thin radial slots in the stainless steel end caps was to use a water jet cutting machine. The cutting machine is capable of cutting slots $0.045 "$ or $1.77 \mathrm{~mm}$ in width. This minimum slot width constraint is then used as the models slot width. Though small, this width is not insignificant and large number of slots (24 slots) which are examined in the study [43] become infeasible when the tops of the slots would connect and cause the top of the end cap to become disconnected from the rest of the end cap. In order to simulate radial slits in an end cap, the model must be made in three dimensions. Using symmetry boundary conditions, the number of elements in the model can be drastically reduced for ease of simulation. For example, the six slit transducer tested

on the MTS machine could be simulated using 12 fold symmetry shown in Figure 3.19 . 


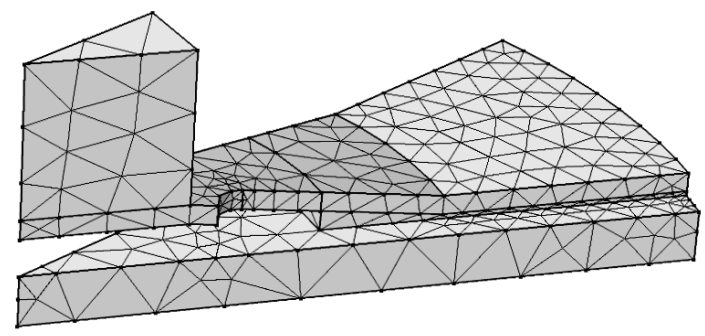

Fig. 3.19: A single twelfth slice of a six slit transducer with a rough mesh for testing model stability .

Displacements of the bottom face as well as the side faces, except for the inside of the slit are constrained to only move in their plane, becoming an effective symmetry boundary condition. The angle of the wedge is calculated as $\theta=180 /$ N_Slits where $\theta$ is the angle in degrees and $N_{-}$Slits is an additional discrete parameter defining the number of slits.

The model was created using the parameters shown in Table 3.3 except for the epoxy ooze depth, which was set to $6.01 \mathrm{~mm}$ to better reflect the actual measurements of the test transducers. The number of radial slits was varied between 2 and 15 radial slits and at four mesh densities to compare the effects of both meshing densities and slit numbers. Due to large stress concentrations near the closed end of the slot, only $0.015 "(0.381 \mathrm{~mm})$ thick stainless steel was considered as an end cap material. This is justified by the preload and end cap FEA studies which found that thinner values of stainless steel end cap are already near their yield load as is shown in Figure 3.7 where only $0.010 "(0.254 \mathrm{~mm})$ and thicker end caps are above its yielding stress. Figure 3.20 shows the results of the slit analysis. The peak total electric energy and vertical displacement both have a positive correlation to slit number. Comparing the material properties in Table 3.2 to the plots in Figure 3.20, we can see that the Piezo material is at all times exceeding its estimated peak yield stress of $2.76 \times 10^{7} \mathrm{~Pa}$. This result indicates that slitting the transducer end caps will have to be accompanied with 
preloading to prevent structural failure in the piezoceramic. Similarly the end cap steel is near its yield limit between 2 to 8 radial slits. As the model approaches 9 to 10 radial slits and above, the peak stresses in the steel begin to exceed its yield stress of $9.65 \times 10^{8} \mathrm{~Pa}$. For safety, it was decided to try four and six slit designs as prototypes as to not over stress the delicate and brittle PZT discs and their end caps.
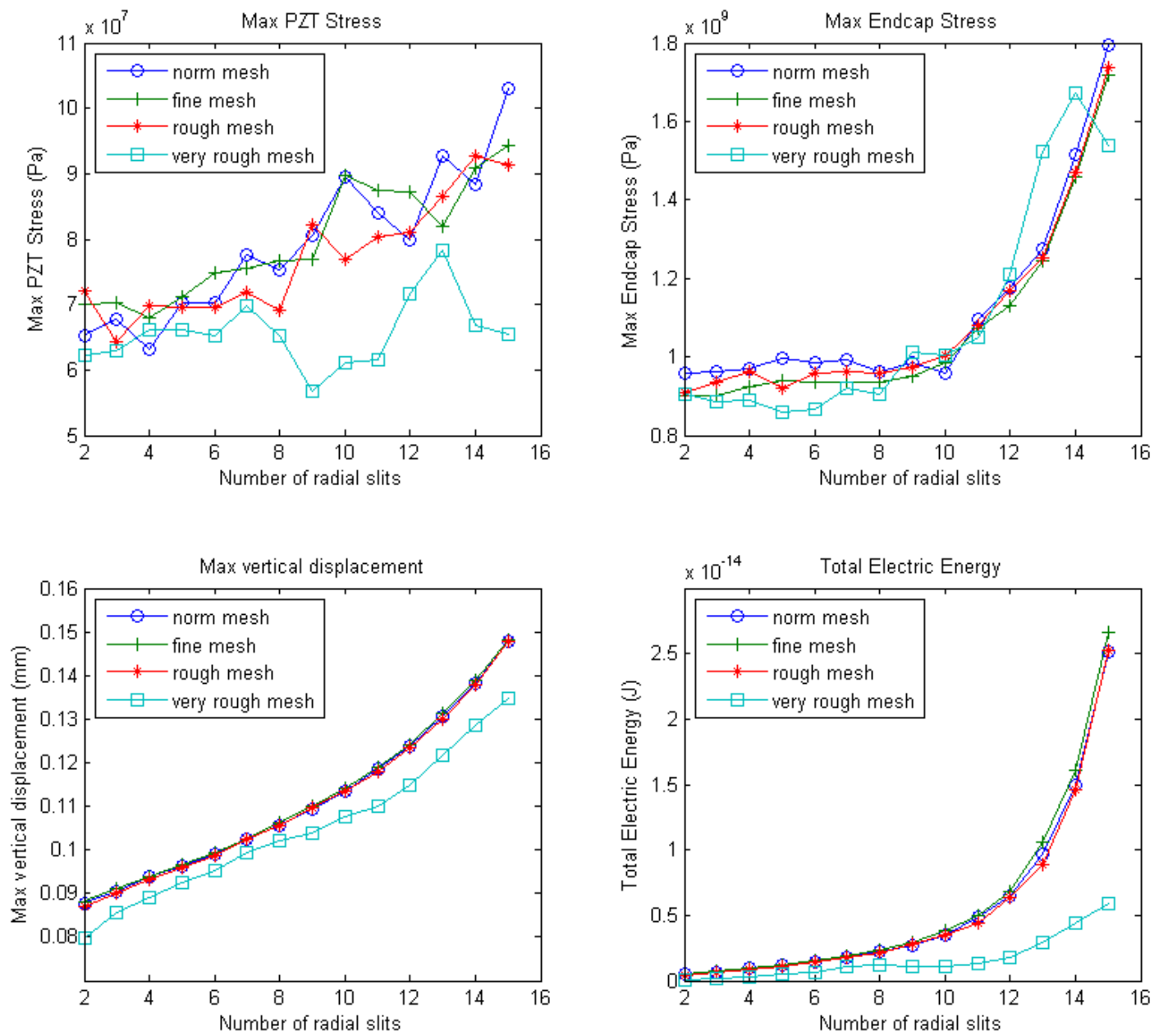

Fig. 3.20: Slit model parametric study results, varying the number of slits.

Figure 3.21 shows the end cap stresses as its thickness is varied with a six slitted transducer model, while Figure 3.22 shows the maximum PZT stresses under the same conditions. From these two plots, it was decided to use the thickest end cap material being considered, $0.381 \mathrm{~mm}$. The $0.381 \mathrm{~mm}$ thick end cap parameter satisfies the reported yield strength of the end cap material 301 stainless steel at $9.65 \times 10^{8} \mathrm{~Pa}$. The 
stresses for the PZT disc, shown in Figure 3.22, exceed the material yield strengths as expected. The added radial slits appropriately amplify the forces on the piezoceramic material which will be compensated for in practice by preloading. A prototype with too many slots may experience yielding or failure within the end caps of the transducer and as a result of these FEA results, it was decided to manufacture four and six slitted end capped prototypes to be compared and preloaded.

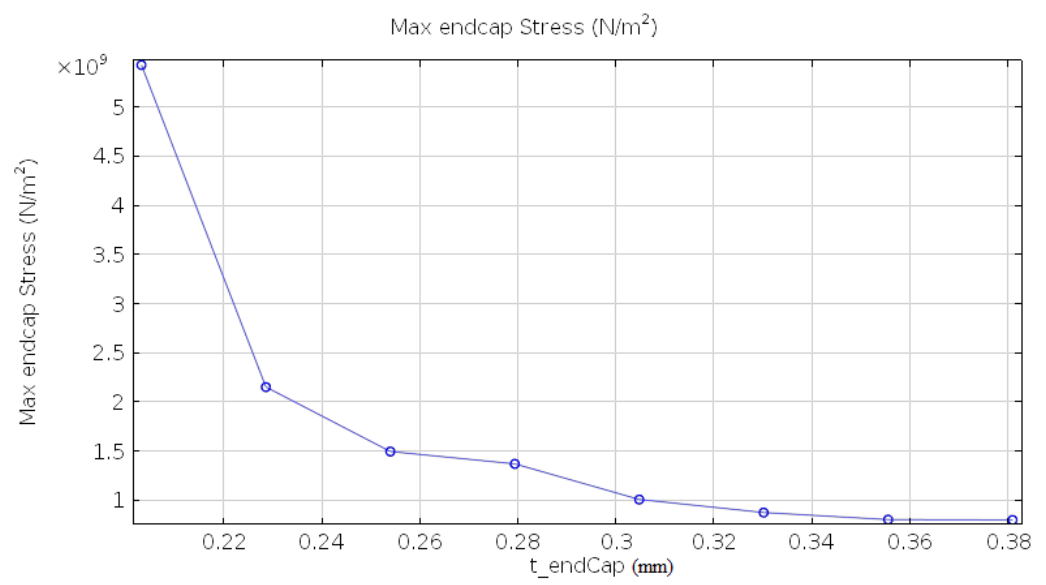

Fig. 3.21: Maximum end cap stresses in the slit model plotted against end cap thickness.

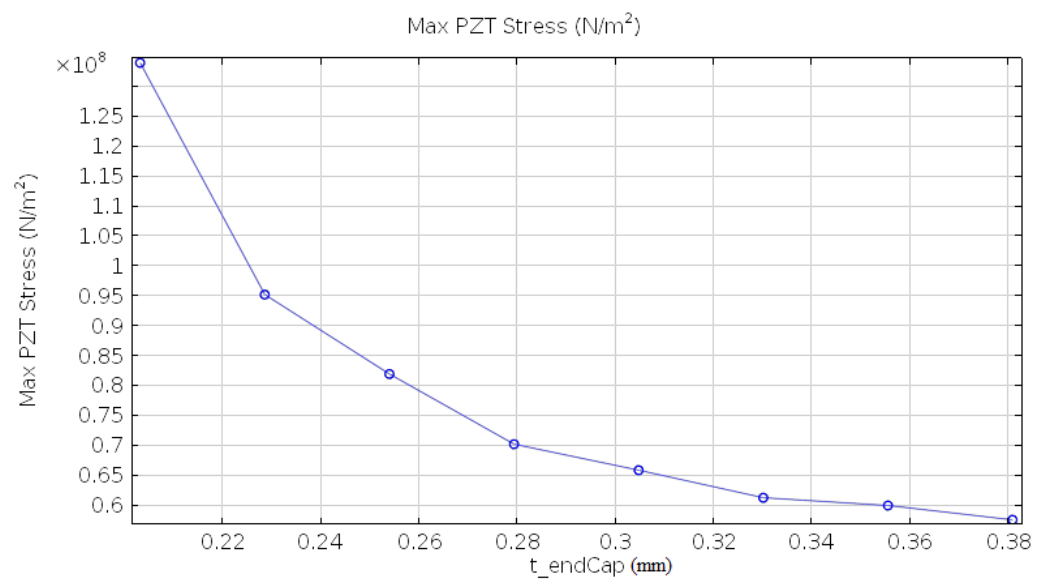

Fig. 3.22: Maximum PZT stresses in the slit model plotted against end cap thickness. 


\subsection{FEA conclusions}

From the work performed in the previous section, it was decided that the final prototypes for the novel transducer design will have to have a $0.015 "(0.381 \mathrm{~mm})$ thick stainless steel end cap because of the materials strength and because of the stress concentrations introduced from adding radial slits. The prototypes must be preloaded using half of the ideal preload tension of $\frac{438.41 \mathrm{~N}}{2}=219.21 \mathrm{~N}$ at full design load of 367.875 N. Given the results and increased stress concentrations from adding radial slits, the final prototypes will have either 4 or 6 radial slots which will be tested and compared as only $0.013 "(0.3302 \mathrm{~mm})$ thick end caps will be able to survive the load with six radial slits. Finally the transducers must be manufactured with as little epoxy ooze as possible to avoid poor transducer performance.

Attempts at creating a model incorporating both radially slitted end caps and preloading caused instabilities. Prototypes of the modeled transducers along with experiments were then used to verify the conclusions made here and are discussed in the following chapter. 


\section{Implementation and testing}

\subsection{Initial transducer designs}

\subsubsection{Bridge transducer}

Initially simple bridge and cymbal transducers were manufactured as were compared in [38]. Figure 4.1 shows a bridge transducer which was constructed for comparison with our early cymbal transducer designs. A potential benefit of the Bridge transducer could be an increased capacity to generate electricity due to a larger amount of piezoceramic material per transducer, which translates into more surface area for charge to be generated, when compared to a cymbal transducer of the same diameter. Another benefit would be fewer power losses due to less strain energy lost to end cap deformation since the end cap material would not have to expand cylindrically like the cymbal transducer. Like [38] however, it was found that the Bridge transducers are much less structurally stable than the Cymbal transducer and that the Bridge transducers were prone to structural failure of the PZT plate.

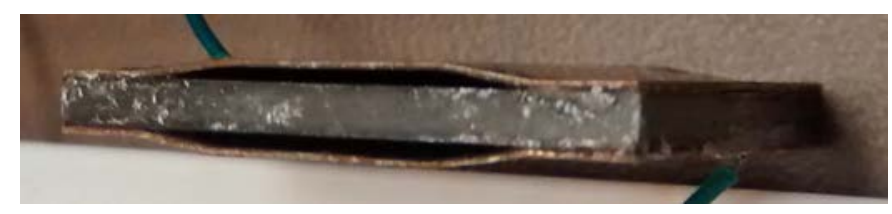

Fig. 4.1: Bridge transducer.

\subsubsection{Cymbal transducer}

Cymbal transducers are manufactured by fabricating metal end caps which are then adhered to a piezoelectric disc. The construction of the end caps and their connection to the PZT discs then becomes the main challenge in the transducers assembly. First, stainless steel or bronze discs are punched out of a sheet using a disc cutter. These 
discs are then fed into a custom machined die attached to a die set shown in Figure 4.2. The additional male and female die in the right hand side of Figure 4.2 are for punching metal squares into bridge transducers. The die is placed in a manual press shown in Figure 4.3 and stamps the metal discs into the desired end cap shape. Finally the completed end caps are adhered to the piezoceramic discs using a stronger non conductive epoxy and a small amount of weaker silver conductive epoxy to improve ease and reliability of wiring.

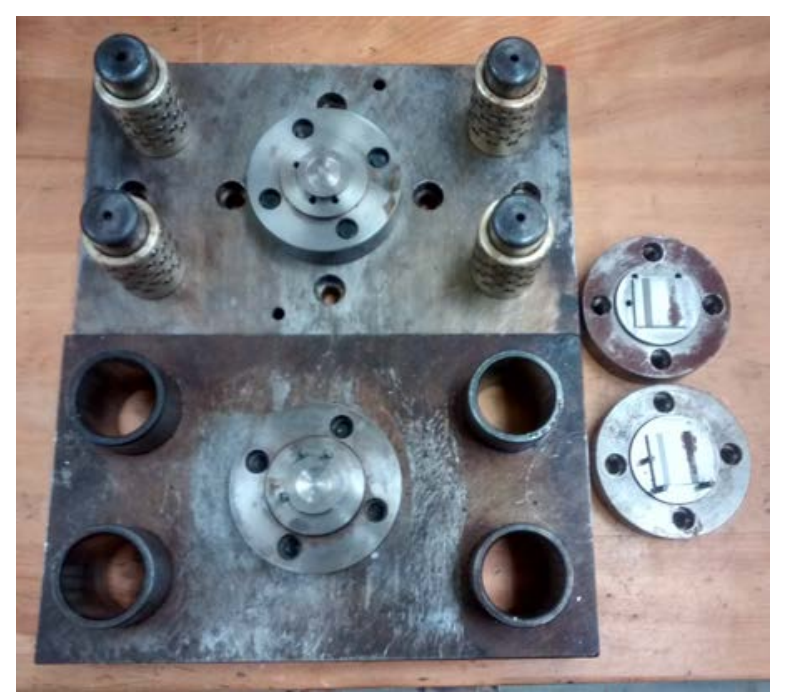

Fig. 4.2: Cymbal end cap male and female dies attached to a die set (centerleft) with bridge dies (right).

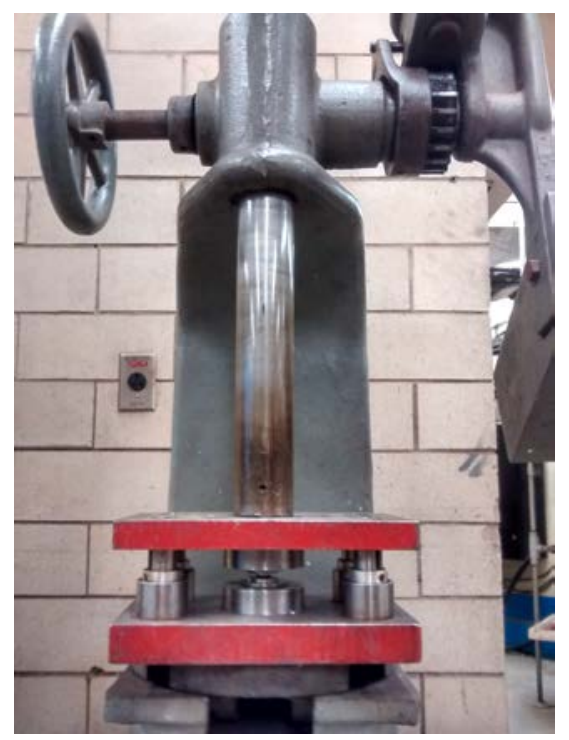

Fig. 4.3: Manual press for operating the die set. 
Once it was decided what type transducer was to be used, a simple harvester was constructed using 9 pairs of Cymbal transducers. Shown in Figure 4.4, the $3 \times 3$ power harvester is composed of 18 transducers in total. The Cymbal transducers stacked in pairs to improve the amount of electric charge generation. The power generated along with the harvesting pad thickness and the amount the pad will be displaced when compressed can be tuned by stacking many transducers vertically. The 1" diameter transducers were designed to collectively harvest power from the full design load of an approximately 1,500 kg vehicle and were placed in a machined nylon block to support and protect the transducers. The nylon block, housing the transducers, included grooves and additional holes machined into it for wiring purposes and for the attachment of diode rectifiers. Nylon was used because of its machinability, low cost, dielectric properties, water resistance, and rigidity. Each transducer pair was connected in parallel and then connected to the main power bus using the diode rectifiers. By isolating the transducer pairs with the rectifiers, charge that would be lost due to the inverse piezoelectric effect caused by uneven loading of the harvesting pad is avoided.

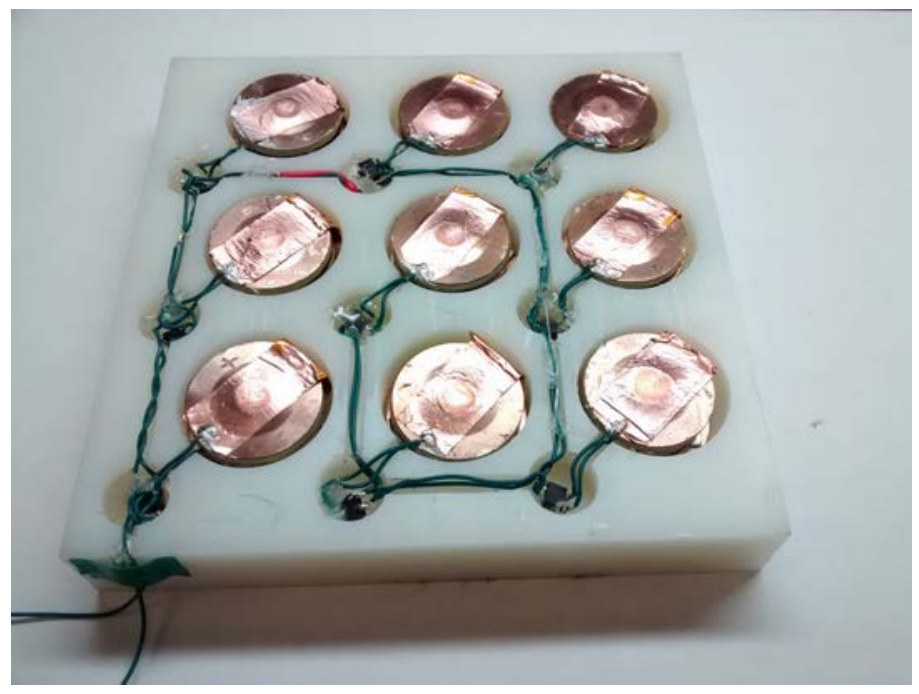

Fig. 4.4: Early tested vehicle harvester.

The depth of the nylon pockets were machined according to FEA estimates of the 
compression displacement of the transducers such that the Cymbal transducers would not be over compressed if the design load was exceeded by a vehicle. The harvester was tested on an MTS ${ }^{\circledR}$ machine, with the test setup being shown in Figure 4.5.

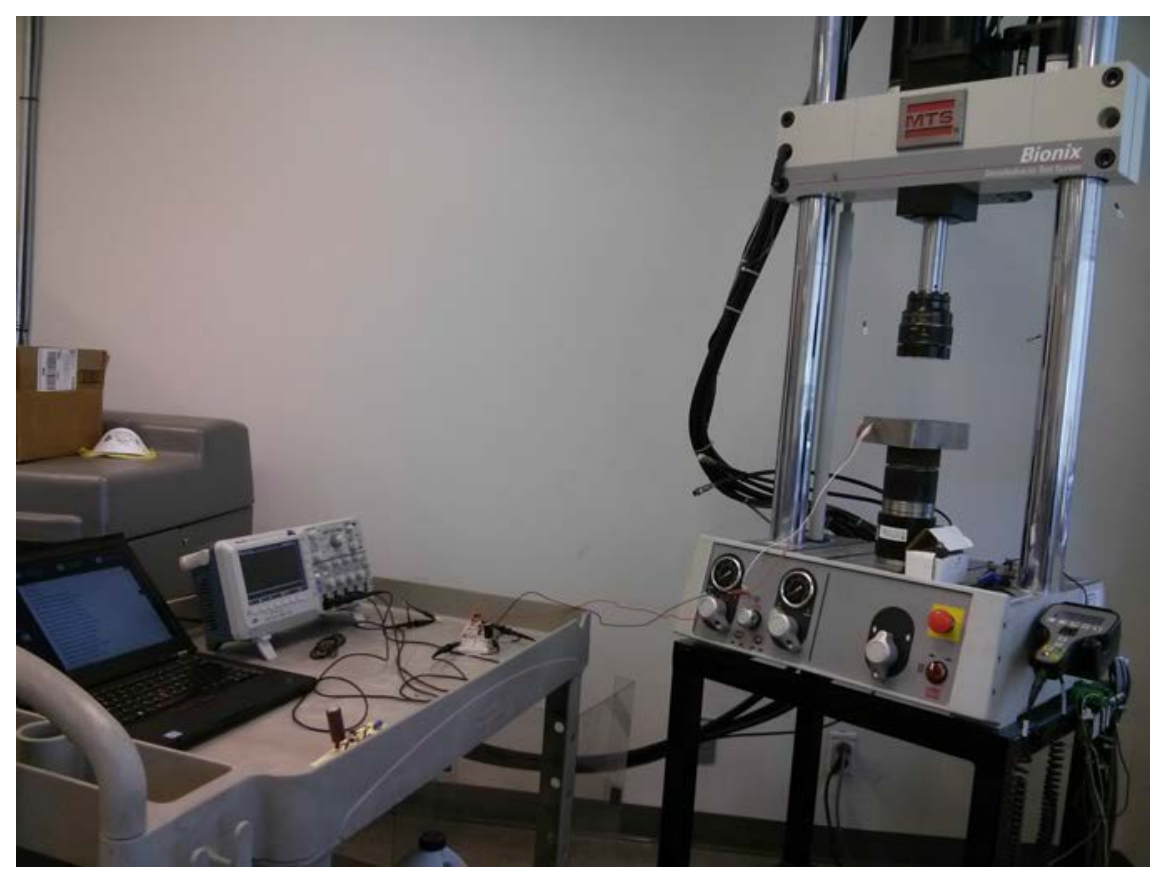

Fig. 4.5: MTS ${ }^{\circledR}$ test setup.

The setup in Figure 4.5 consisted of the $\mathrm{MTS}^{\circledR}$ machine with a custom mounting bracket to hold and contain the harvester pictured on the right of the image. The custom mounting bracket is placed on top of a load cell for verification of the loading force. A Tektronix ${ }^{\circledR}$ DPO 2024B oscilloscope (shown on the left) was used to make measurements of the harvesters voltage and various power harvesting circuits being worked on while tests were under way. As a baseline, the voltage of the harvester was measured at different loading forces and frequencies. Ideally the measurements would represent open circuit voltages however the oscilloscope probes have a $10 \mathrm{M} \Omega$ resistance and acted as a $10 \mathrm{M} \Omega$ load which decays the voltage trace accordingly.

Peak voltage is used in all of the tests as a performance metric. This is because of how a harvesting circuit connected to the system is intended to work. Chapter 2: Power 
harvesting electronics outlines a harvesting circuit model which makes use of peak voltage detection. When this circuit detects a peak voltage, the energy stored in the piezoelectric material is discharged into the circuit. The amount of energy available to the circuit for harvesting is represented by Equation 2.2, $E=\frac{1}{2} C V^{2}$, which is a function of peak transducer voltage and its capacitance. Since the piezoelectric discs, and therefore the transducer capacitance, are standardized between transducers, then the peak voltage becomes a sole metric for transducer performance.

Figure 4.6 shows the traces produced by the harvester at 1 and $2 \mathrm{kN}$ loads for excitation frequencies $0.25,0.5$, and $1 \mathrm{~Hz}$. The peak voltages of these traces where measured and are displayed in Table 4.1. A simple equation for estimating power consumed, or more accurately power dissipated by a load is proposed in [50] and is shown in Equation 4.1.

$$
P=\frac{V_{r m s}^{2}}{R}
$$
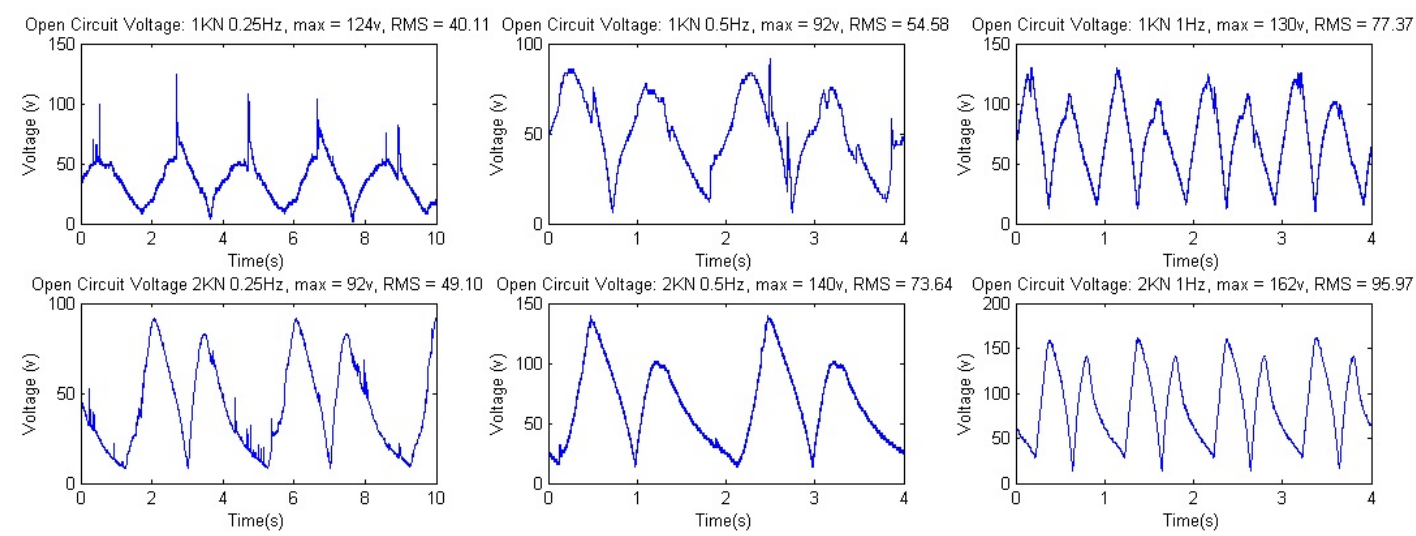

Fig. 4.6: Example harvester traces measured during the MTS ${ }^{\circledR}$ tests. 
Table 4.1: Harvester MTS ${ }^{\circledR}$ machine test peak voltage results.

\begin{tabular}{|c|c|c|c|}
\hline & $0.25 \mathrm{~Hz}$ & $0.5 \mathrm{~Hz}$ & $1 \mathrm{~Hz}$ \\
\hline $1 \mathrm{kN}$ & $124-66 \mathrm{~V}$ & $92-84 \mathrm{~V}$ & $130-124 \mathrm{~V}$ \\
\hline $2 \mathrm{kN}$ & $92-92 \mathrm{~V}$ & $140-140 \mathrm{~V}$ & $162-160 \mathrm{~V}$ \\
\hline $3 \mathrm{kN}$ & - & - & $162-160 \mathrm{~V}$ \\
\hline
\end{tabular}

Using Equation 4.1 and the oscilloscope probe resistance, the dissipated power is calculated and tabulated in Table 4.2. This power represents the amount of available electrical power that would be available to a load connected directly to the piezoelectric harvesting pad.

Table 4.2: Harvester MTS ${ }^{\circledR}$ machine test power dissipation results.

\begin{tabular}{|c|c|c|c|}
\hline & $0.25 \mathrm{~Hz}$ & $0.5 \mathrm{~Hz}$ & $1 \mathrm{~Hz}$ \\
\hline $1 \mathrm{kN}$ & $0.161 \mathrm{~mW}$ & $0.298 \mathrm{~mW}$ & $0.599 \mathrm{~mW}$ \\
\hline $2 \mathrm{kN}$ & $0.241 \mathrm{~mW}$ & $0.542 \mathrm{~mW}$ & $0.921 \mathrm{~mW}$ \\
\hline $3 \mathrm{kN}$ & - & - & $0.984 \mathrm{~mW}$ \\
\hline
\end{tabular}

\subsection{Comparison of slitted and solid endcapped transducers}

In order to measure the performance of novel transducer designs compared with conventional designs, a series of iterative experiments were performed on an $\mathrm{MTS}^{\circledR}$ machine pictured in Figure 4.5.

\subsubsection{Initial feasibility testing}

The initial experiments which were performed with individual transducers on the the MTS ${ }^{\circledR}$ machine setup shown in Figure 4.5, set up to use a force feedback mode to apply a $0 \mathrm{~N}$ to $400 \mathrm{~N}$ sinusoidal load, above the $367.875 \mathrm{~N}$ design load in order to test the transducers at their feasible limits. Each transducer was inserted into a cast and machined nylon housing to protect it during testing (shown in Figure 4.7) and placed 
on top of the custom bracket used in earlier tests. The rightmost block in Figure 4.7 is used for simple cymbal transducers with and without radial slitting and preloading, and the center container is used to test cymbal transducers with preloading which require more space. The cover for the blocks, on which the load is applied is shown on the left.

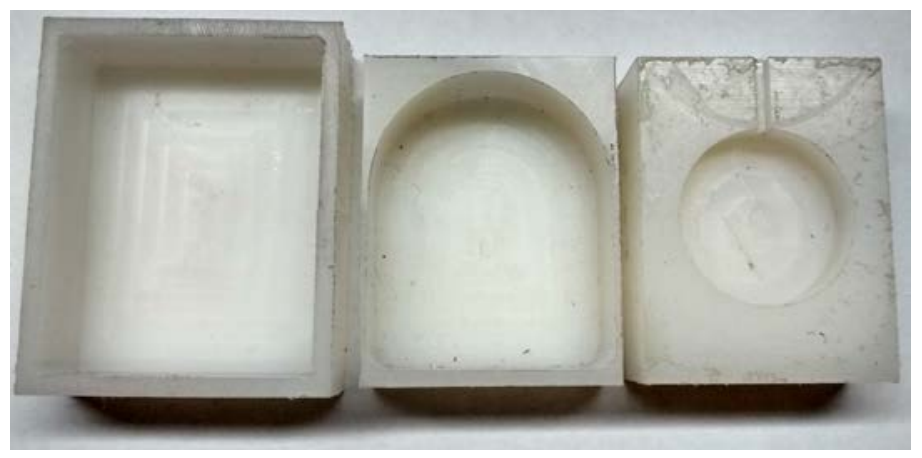

Fig. 4.7: Machined cast nylon individual transducer test housing.

In order to compare the differences between a solid endcapped transducer and one with four slits each, two pairs, for a total of four transducers were manufactured with one pair having four radial slits in its end caps and the other with non-slitted endcaps. One transducer from each pair is shown in Figure 4.8). The four 0.015" (0.381 mm) thick end caps were made with 301 stainless steel with material properties shown in Table 3.2. One transducer from each pair was adhered to the piezoceramic disc using Devcon ${ }^{\circledR} 5$ minute ${ }^{\circledR}$ epoxy gel, and were labeled "Slitted" and "solid" "Transducer 1" while the other two were attached using Lepage ${ }^{\circledR}$ professional speed set $^{T M}$ epoxy, and labeled "Slitted" and "solid" "Transducer 2", to examine the effects of different epoxies on the transducer performances. The cycling frequency was set to $0.25 \mathrm{~Hz}$ as the transducers are expected to operate in a low frequency manner of a traversing vehicle every few seconds. The Cymbal units were connected directly to the oscilloscope to measure the resulting voltage. 


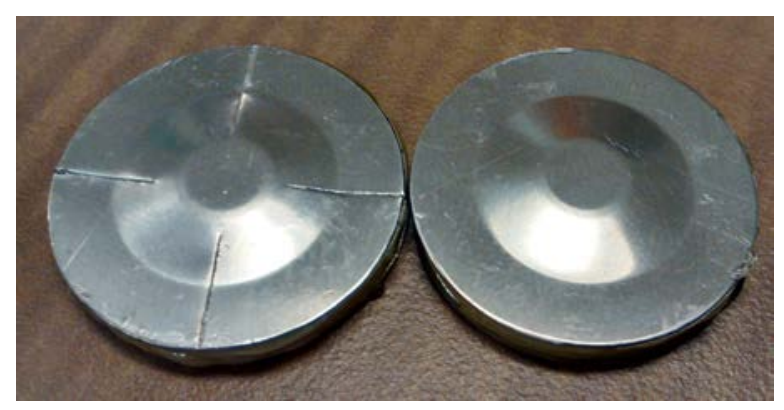

Fig. 4.8: Image of a slitted (left) and solid (right) transducers assembled before wiring and testing.

\subsubsection{Feasibility testing results}

The results of the experiment are shown in Table 4.3. Performance of "Slitted Transducer 2" was higher in terms of peak output voltage, reaching $39.8 \mathrm{~V}$, than both solid transducers at the same $34.2 \mathrm{~V}$ level. The other slitted transducer performed worse than all the others with a peak of $17.0 \mathrm{~V}$. This disparity indicates that there are more significant factors in the peak transducer output voltage than the number of radial slits in the end caps. In order to examine some of the important factors to performance, the over performing slitted transducer was disassembled and shown in Figure 4.9. The transducer was damaged after the test by an erroneous $\mathrm{MTS}^{\circledR}$ machine command, however this did not affect the experimental results. Comparison of the notable differences between the under performing slitted transducer (shown in Figure 4.10) and the others revealed that it had:

- Less epoxy between end caps and PZT disc resulting in less oozing into the center cavity.

- The epoxy did ooze inward in some spots, but the epoxy penetration depth into the cymbal was inconsistent around the cymbal shown in Figure 4.9 (especially near the bottom) 
- Used Lepage ${ }^{\circledR}$ professional speed $\operatorname{set}^{\mathrm{TM}}$ epoxy

- The epoxy thickness looked consistently small between the outer edge of the transducer and the end cap

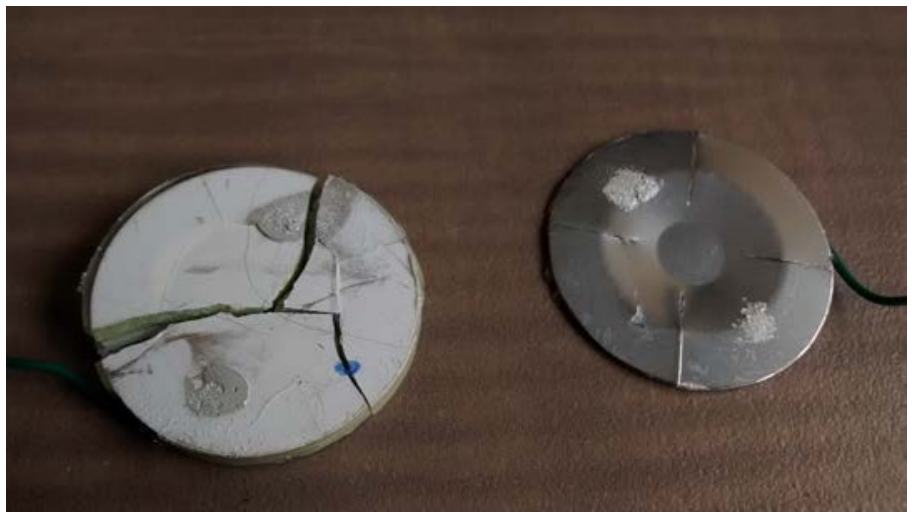

Fig. 4.9: The over performing transducer, "Slitted Transducer 2".

The other transducer, "Slitted Transducer 1", shown in Figure 4.10 by contrast to the other transducers had:

- More epoxy ooze toward the central cavity

- Was manufactured with more consistent epoxy placement

- Used Devcon ${ }^{\circledR} 5$ minute epoxy gel

- Epoxy was much thicker in some areas causing small epoxy filled gaps between the end cap and piezo disc 


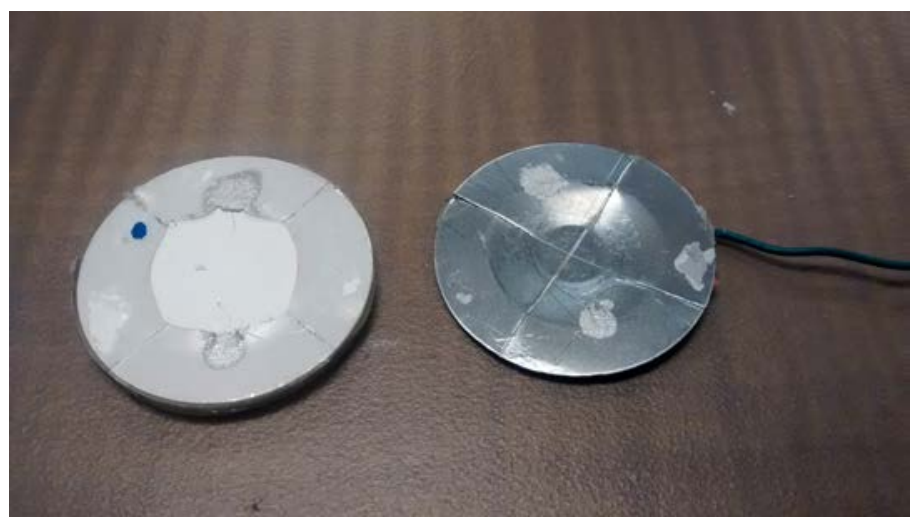

Fig. 4.10: The under performing transducer (Taken apart after the test), "Slitted Transducer 1".

Analysis was performed on the oscilloscope trace data using MATLAB ${ }^{\circledR}$. The transducers were connected to the oscilloscope directly through $10 \mathrm{M} \Omega$ leads. The calculated power dissipated through the traces (shown in Figure 4.11) according to Equation 4.1, as well as the peak voltage attained is shown in Table 4.3. The over performing slitted transducer is labeled "Slitted Transducer 2" in Table 4.3 while the under performing one is labeled as "Slitted Transducer 1".

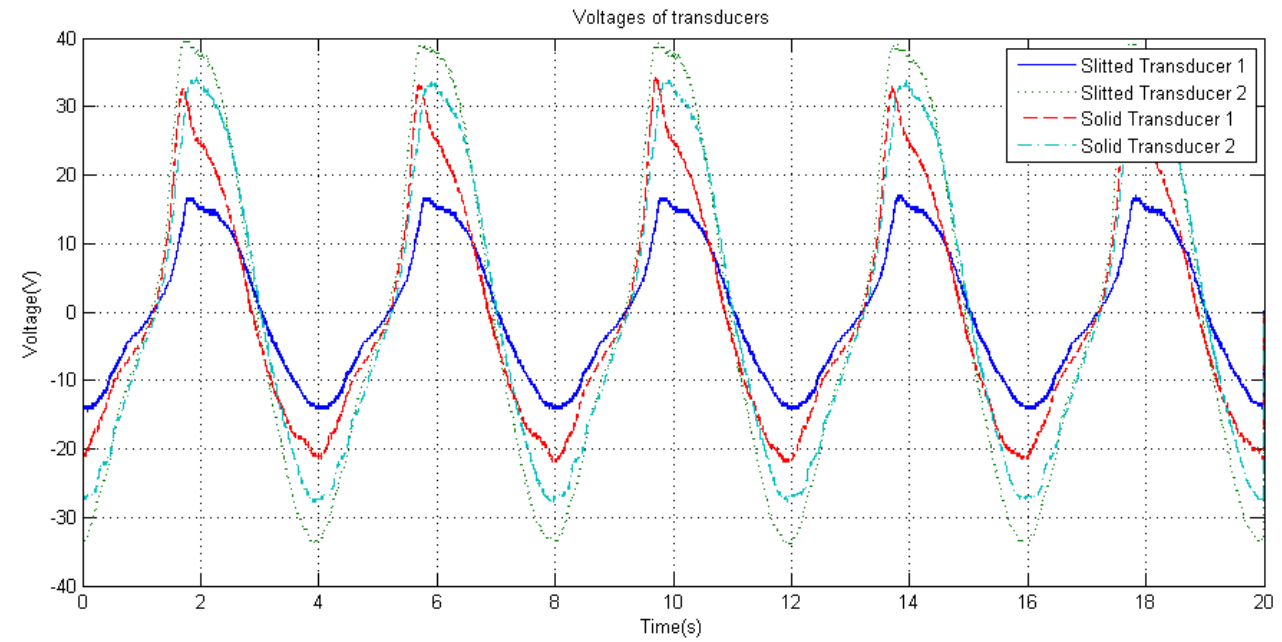

Fig. 4.11: Traces of tested transducers during 1st test. 
Table 4.3: Power dissipated by the oscilloscope, produced by transducers in the 1st experiment.

\begin{tabular}{|c|c|c|}
\hline Transducer & Power $(\mu \mathrm{W})$ & Peak voltage $(\mathrm{V})$ \\
\hline \hline Slitted Transducer 1 & 10.0 & $17.0-16.6$ \\
\hline Slitted Transducer 2 & 60.3 & $39.8-39.0$ \\
\hline Solid Transducer 1 & 26.6 & $34.2-32.6$ \\
\hline Solid Transducer 2 & 42.1 & $34.2-33.8$ \\
\hline
\end{tabular}

The two solid transducers have been manufactured the same ways as the slitted transducers however one dissipated $58.6 \%$ more power than the other because it sustained a high voltage after each voltage peak. This indicates that the Epoxy contributes to the transducer charge dissipation performance. Part of the difference is also attributable to manufacturing consistency. Both of the solid transducers had a large amount of epoxy ooze into the cavity in the end cap with Solid transducer 1 (shown in Figure 4.12) showing slightly less ooze than Solid transducer 2 (shown in Figure 4.13). This indicates that the ooze depth may also be the cause of reduced power generation as predicted by the epoxy ooze FEA study.

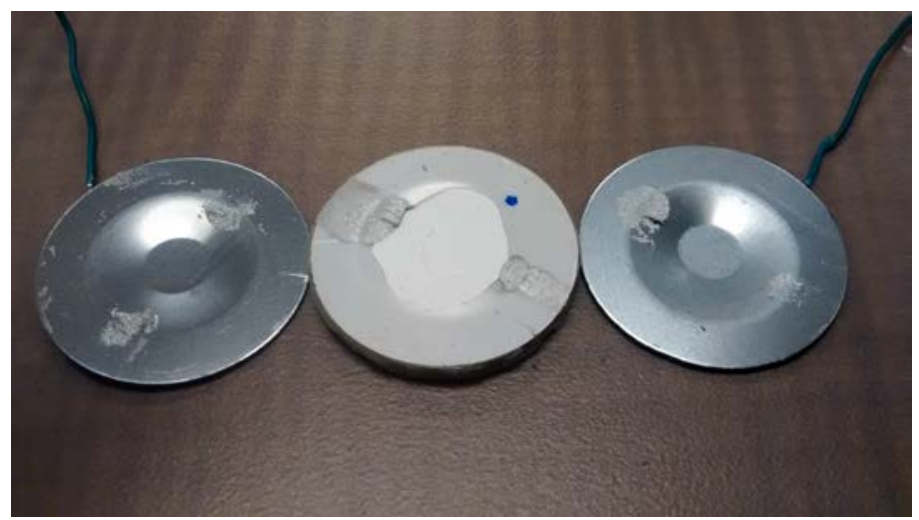

Fig. 4.12: One of the solid end capped transducers (Solid Transducer 1), taken apart after the test. 


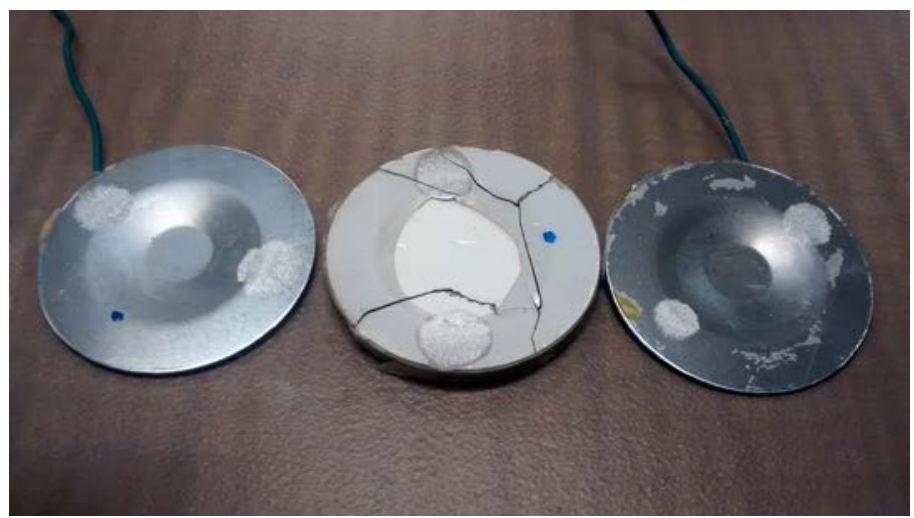

Fig. 4.13: The other solid end capped transducer (Solid Transducer 2) taken apart after the test (The PZT material cracked during disassembly).

\subsubsection{Epoxy ooze experiment}

To confirm the results of the FEA ooze parametric study, one solid and two slitted transducers were built and shown in Figure 4.14. In order to attempt to get more consistent and improved peak voltage, the harvesting units were built with less epoxy. This test only used the Lepage ${ }^{\circledR}$ professional speed set $^{\mathrm{TM}}$ epoxy which was applied near the outside rim of the transducer end cap in order to reduce the amount of ooze into the center cavity. During testing, both slitted transducers became partially detached from the PZT material affecting the final results and implying that too little of an amount of epoxy was used and affected the resulting output power. The solid transducer did not have this issue and was not damaged during the test. 


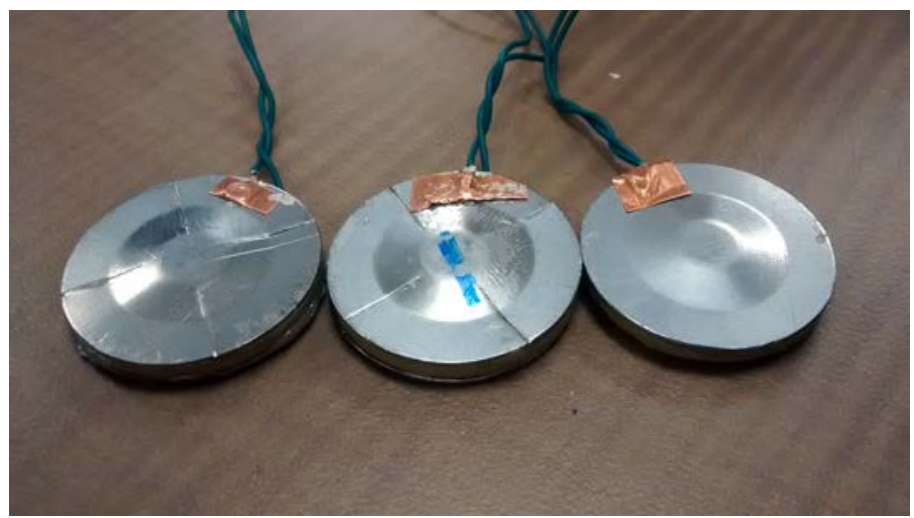

Fig. 4.14: The rebuilt transducers from left to right: Slitted Transducer 2, Slitted transducer 1, Solid traducer.

The traces collected for the epoxy ooze experiment are shown in Figure 4.15 and shows a very large improvement in performance for the solid end capped transducer which reached a peak voltage of $57.6 \mathrm{~V}$.

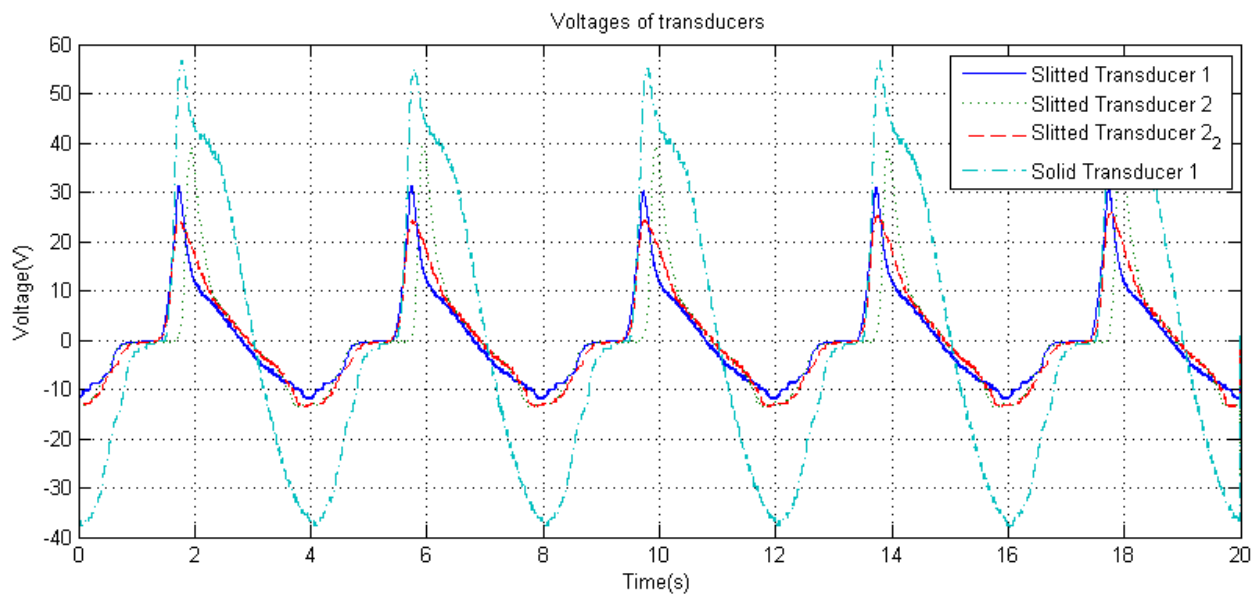

Fig. 4.15: Traces from the epoxy ooze MTS ${ }^{\circledR}$ test.

The peak voltage and power dissipation results are shown in Table 4.4 which are calculated in similar fashion to the previous experiment, using Equation 4.1. 
Table 4.4: Power produced by the rebuilt transducers.

\begin{tabular}{|c|c|c|}
\hline Transducer & Power $(\mu \mathrm{W})$ & Peak voltage (V) \\
\hline \hline Slitted Transducer 1 & 8.78 & $31.2-30.4$ \\
\hline Slitted Transducer 2 & 12.6 & $39.6-38.8$ \\
\hline Slitted Transducer 2 (run 2) & 10.1 & $26.0-24$ \\
\hline Solid Transducer & 76.6 & $57.6-55.2$ \\
\hline
\end{tabular}

The failure of both slitted transducers as the $400 \mathrm{~N}$ load was applied repeatedly is the reason the power produced by both slitted transducers appears low compared to the solid transducer in the experimental results in Table 4.4. Also, it is noted that the solid transducer tested in this experiment produced a higher peak voltage than the peak voltage from "Slitted Transducer 2", reaching $57.6 \mathrm{~V}$ compared to the $39.8 \mathrm{~V}$ measured during the previous test. This further indicates that manufacturing inconsistency is the cause of the experimental result inconsistency of the transducers. Images of the disassembled transducers are shown in Figures 4.16, 4.17, and 4.18 and show inconsistent epoxy coverage as well as excessively applied conductive epoxy. Meanwhile, the solid transducer that performed very well shows appropriately small conductive epoxy application as well as a much more consistent and appropriate amount of non-conductive epoxy.

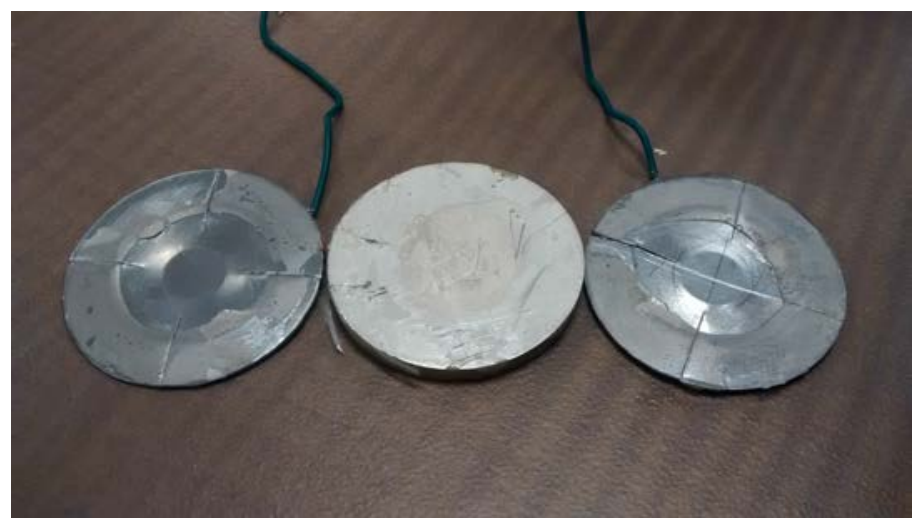

Fig. 4.16: Slitted Transducer 1 after testing. The break seems to be caused by the small patch without epoxy near the left edge of the end cap on the right. 


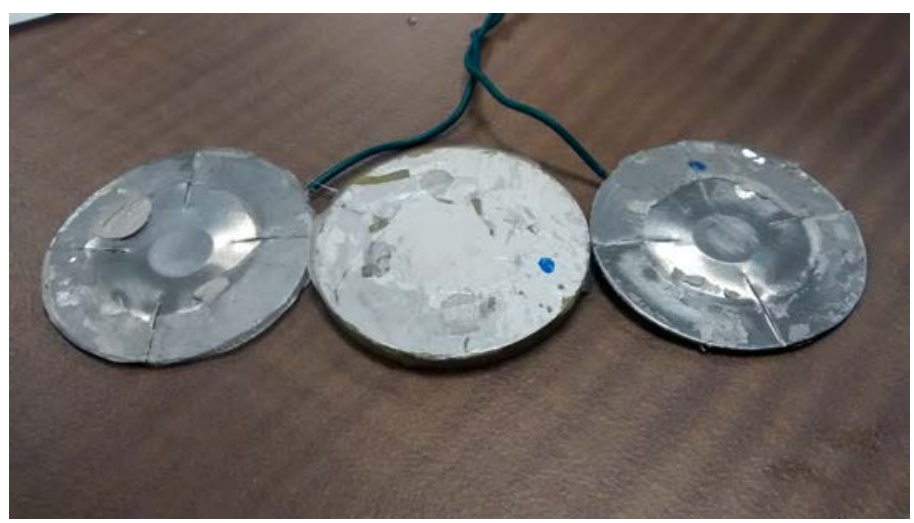

Fig. 4.17: Slitted Transducer 2 after testing. The failure in this case seems to be caused by the larger lump of conductive epoxy near the top left of the left hand end cap.

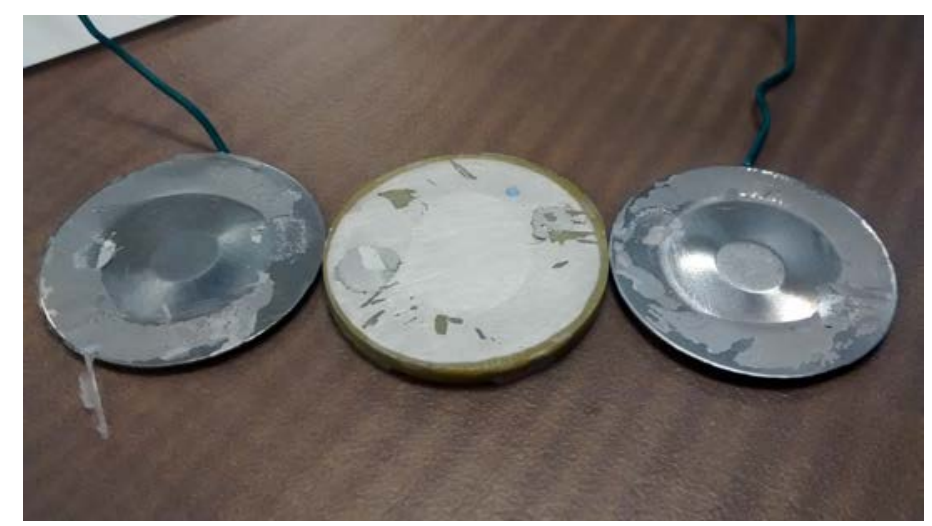

Fig. 4.18: The solid transducer that performed very well and did not fail during the test despite small conductive epoxy ooze seen on the left end cap.

\subsubsection{Initial prestressed and water cut transducers}

In order to improve $\mathrm{MTS}^{\circledR}$ experimental consistency, an improved slitted end cap design and manufacturing process was set up which used water jet cutting to cut $0.045 "$ or $1.77 \mathrm{~mm}$ wide radial slits. Preloaded transducers are able to tolerate higher forces on them by reducing the peak stress in the piezo and epoxy material however this will be explored later. Figure 4.19 shows the water cut transducer end caps to be tested which will produce more consistent results. These water cut end caps are similar to the punched variants except that the discs are cut via water jet. After 
cutting, the discs are pressed in the cymbal die in order to give them their central cavity. Six slits was chosen from the FEA analysis in the previous chapter as well as four slit design to examine the difference and potential experimentally.

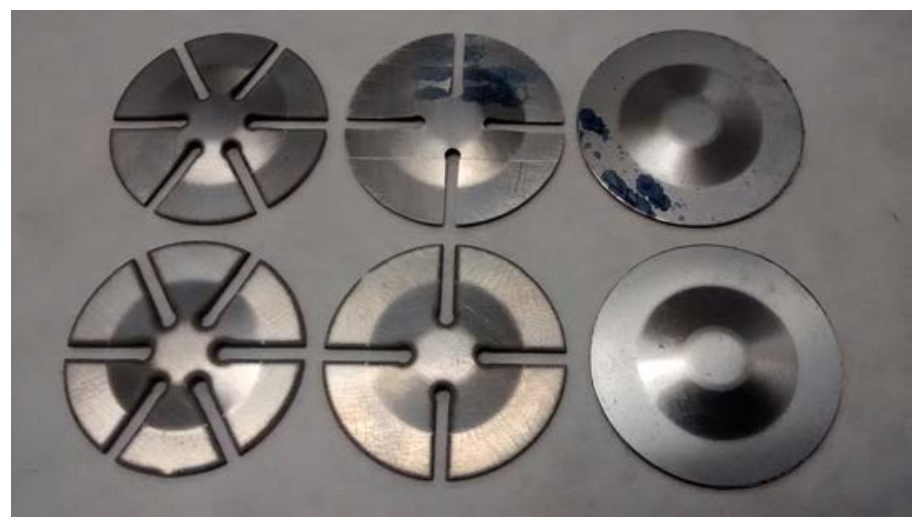

Fig. 4.19: Six and four slit transducer end caps produced by water jet cutting compared to the plain versions on the right.

In order to test the damage resistant properties of preloading, four old phosphor bronze transducers were used and are shown in Figure 4.20. Both preloaded and non preloaded bronze transducers were subjected to an increasing load applied by the $\operatorname{MTS}^{\circledR}$ machine until they reach their failure point. The bronze transducers on the left of Figure 4.20 has an end cap thickness of $0.013 "(0.330 \mathrm{~mm})$ and the two in the center have a thickness of 0.017 ' $(0.432 \mathrm{~mm})$. The two bronze transducers along the top of the image are preloaded and the bottom units are not preloaded. If the preloading works and is applying sufficient pressure, the preloaded transducers will take measurably more load to fail, while the non preloaded transducers will yield more readily. On the right side of Figure 4.20 stainless steel transducers with four and six slits are also prepared for testing. These slitted transducers use $0.015 "(0.381 \mathrm{~mm})$ 301 stainless steel for their end caps following the FEA study suggestion. 


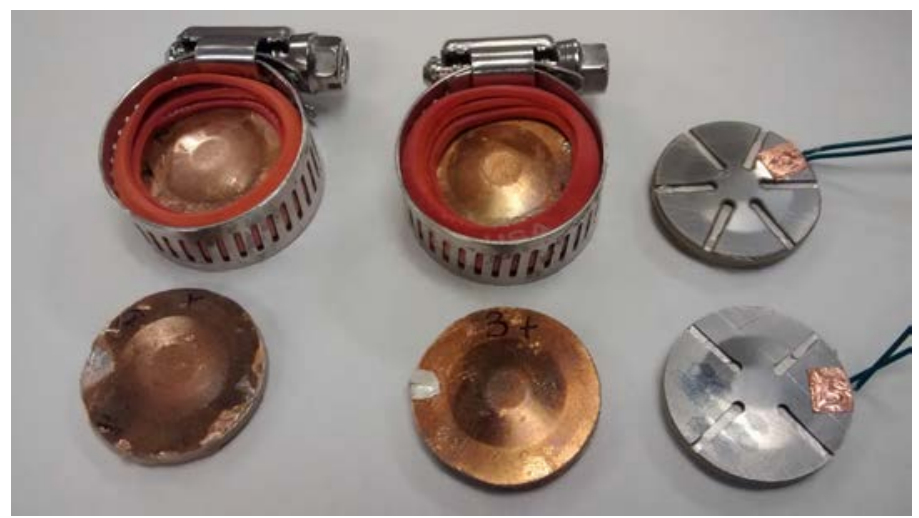

Fig. 4.20: Preloaded transducers next to their non-preloaded counterparts (left) and assembled water cut end cap slitted transducers (right).

Figure 4.21 shows the trace of the four slitted transducer which is shown in the bottom right of Figure 4.20. The transducer performed well compared to earlier transducers, dissipating $127.9 \mu \mathrm{W}$ of power and reaching $89.2-86.0 \mathrm{~V}$ at its peak.

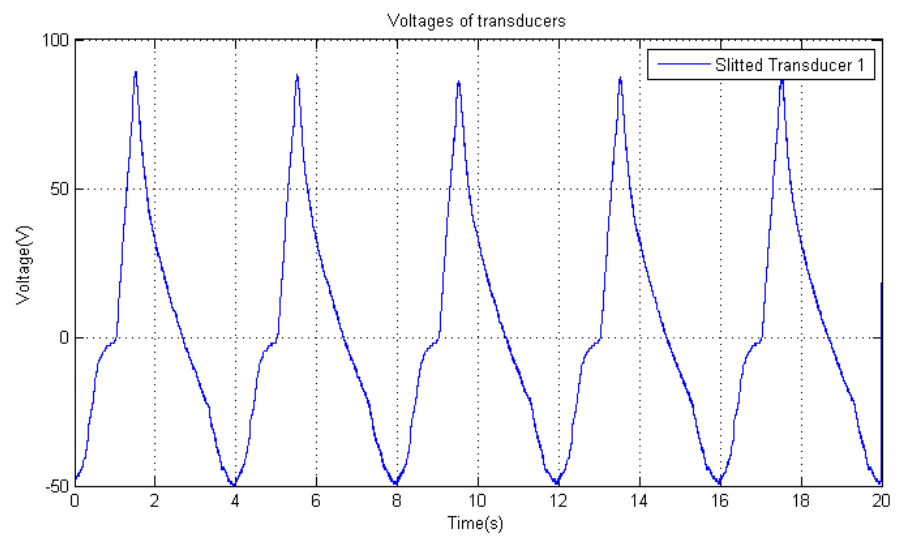

Fig. 4.21: Trace of the four slit transducer.

The six slitted transducer's PZT disc fractured during the test and is shown disassembled after the test in Figure 4.22. The failure of the piezoceramic indicates that the radial slits are amplifying the radial stresses applied to the piezo material significantly more when compared to the other transducers tested, to the point of failure. Following tests try to incorporate preloading in order to prevent such failures and generate more power than the four slitted transducer design. 


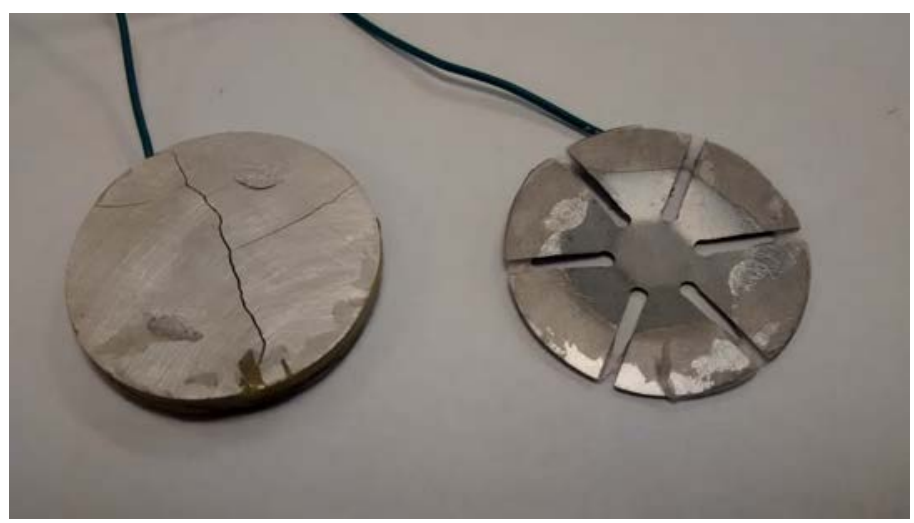

Fig. 4.22: The six slitted transducer after testing.

In order to test the effect of preloading on the failure load, an applied load was slowly increased for the bronze solid endcapped transducers. Failure was estimated through a combination of sharp force drop off detected by the MTS ${ }^{\circledR}$ machine's load cell and listening for sharp cracking sounds indicating piezoceramic or epoxy failure. The preloaded transducer with the $0.13 "(0.330 \mathrm{~mm})$ thick end cap was accidentally loaded to $5 \mathrm{kN}$ (shown in Figure 4.23) and so the specific force when failure occurred could not be accurately attained.

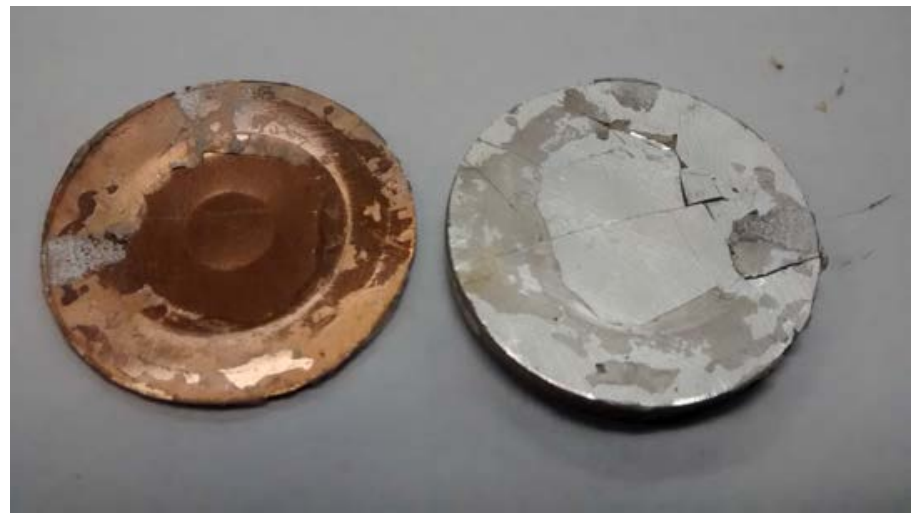

Fig. 4.23: Preloaded transducer after $5 \mathrm{KN}$ load.

Screen shots of the output of the MTS ${ }^{\circledR}$ machine were taken (shown in Figure 4.24 and 4.25) in order to show the loads at the suspected failure points of the preloaded and non preloaded transducers. Failure points were identified by a sharp increase in 
load on the MTS ${ }^{\circledR}$ load cell combined with an auditory cracking sound. Listening for the cracking sound during the experiment was critical to estimating the failure point, especially for the preloaded transducers which produced noisy feedback in the load cells. Shown in Figures 4.24 and 4.25 are the outputs of the $\mathrm{MTS}^{\circledR}$ machine during suspected failure points in the preloaded and non preloaded cymbal transducers with the $0.017 "$ (0.432 mm) thick bronze end caps. The non-preloaded transducer appears to fail at about $605 \mathrm{~N}$ of load force while the preloaded version fails at a much higher $1165 \mathrm{~N}$ or so of load force. The results for the thinner $0.013 "(0.330 \mathrm{~mm})$ thick end capped transducers are less clear.

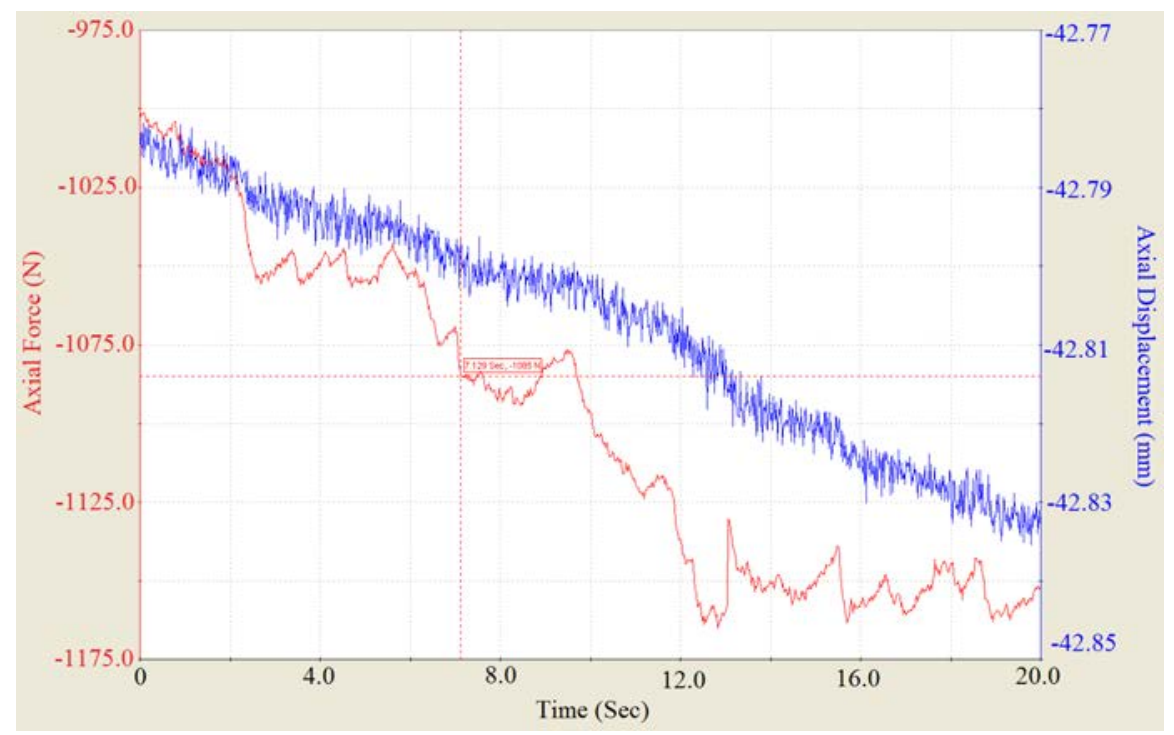

Fig. 4.24: Failure of the preloaded cymbal transducer with $0.017^{\prime \prime}(0.432 \mathbf{m m})$ thick end caps. 


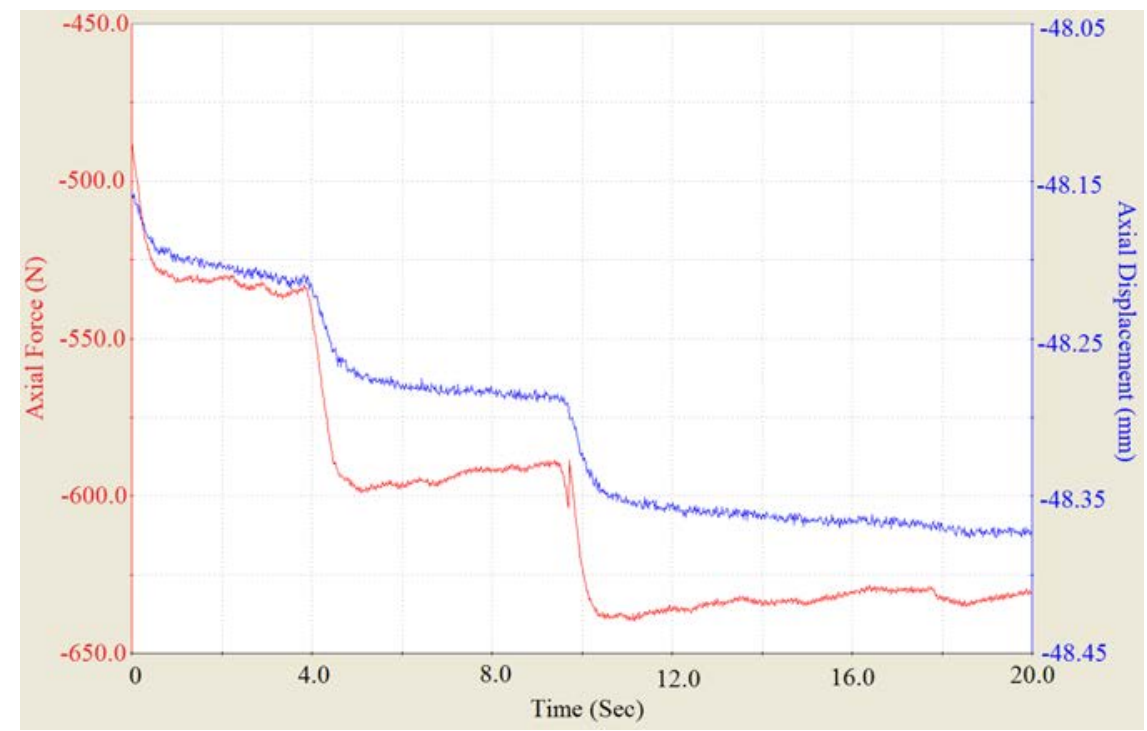

Fig. 4.25: Failure of the non-preloaded cymbal transducer with 0.017 " $(0.432 \mathrm{~mm})$ thick end caps.

Figures 4.26, 4.27, and 4.28 show the results of the thinner 0.013 " thick end cap transducers during their failure $\mathrm{MTS}^{\circledR}$ test. There appeared to be two failure points for the non-preloaded transducer. One failure point was at $975 \mathrm{~N}$ and the other was around $1156 \mathrm{~N}$ of load. It is possible that the transducer failed in several spots during the test in which case the lowest failure point should be chosen. The preloaded version of the transducer was accidentally loaded very quickly as can be seen in the bottom image of Figure 4.28. This accidental destruction of the test transducer, did not prevent the measurement of a result which reached 4910 N. It may be the case, similar to that of its non-preloaded counterpart, that the preloaded cymbal failed at several load levels, however the loading rate was too high or the failure signs too subtle, for the instrumentation to pick up on them. 


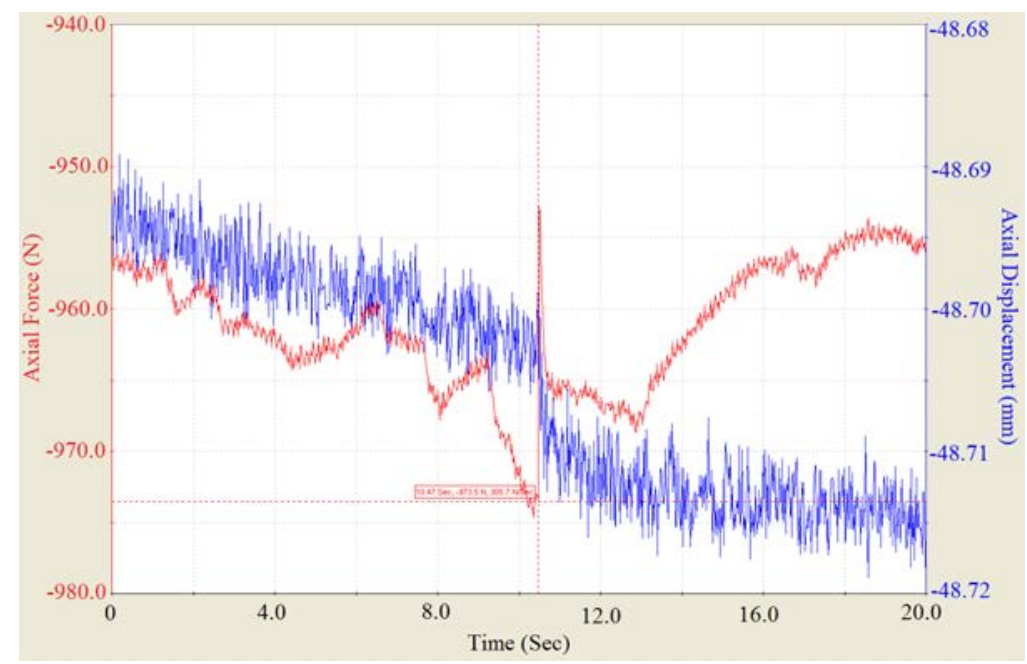

Fig. 4.26: First failure of the non-preloaded, $0.013 "(0.330 \mathrm{~mm})$ transducer.

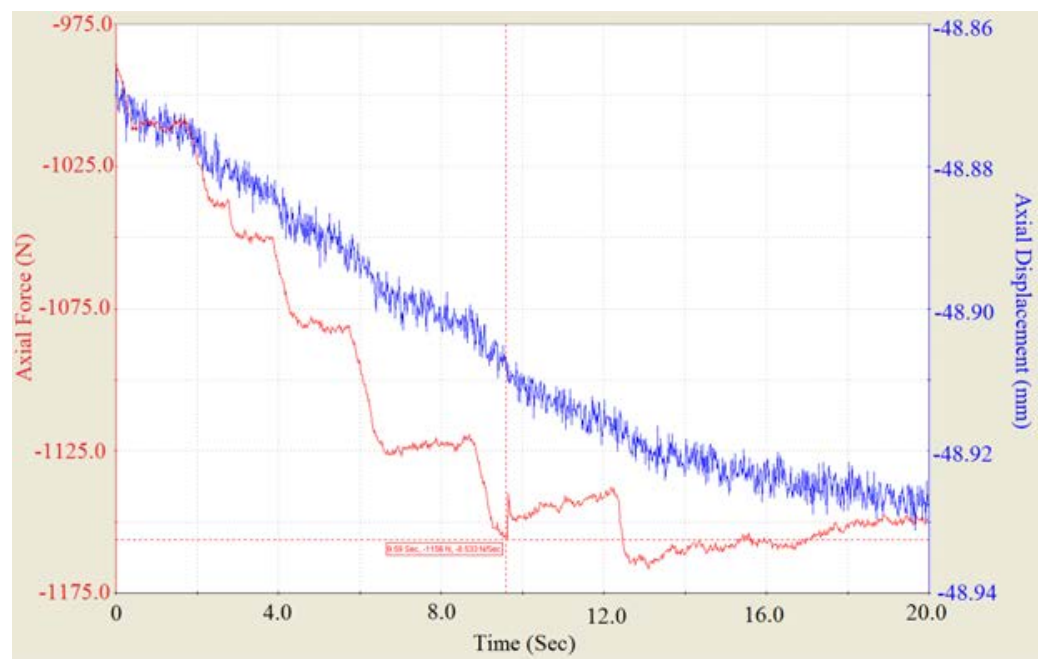

Fig. 4.27: Second failure of the non-preloaded, $0.013 "(0.330 \mathrm{~mm})$ transducer. 


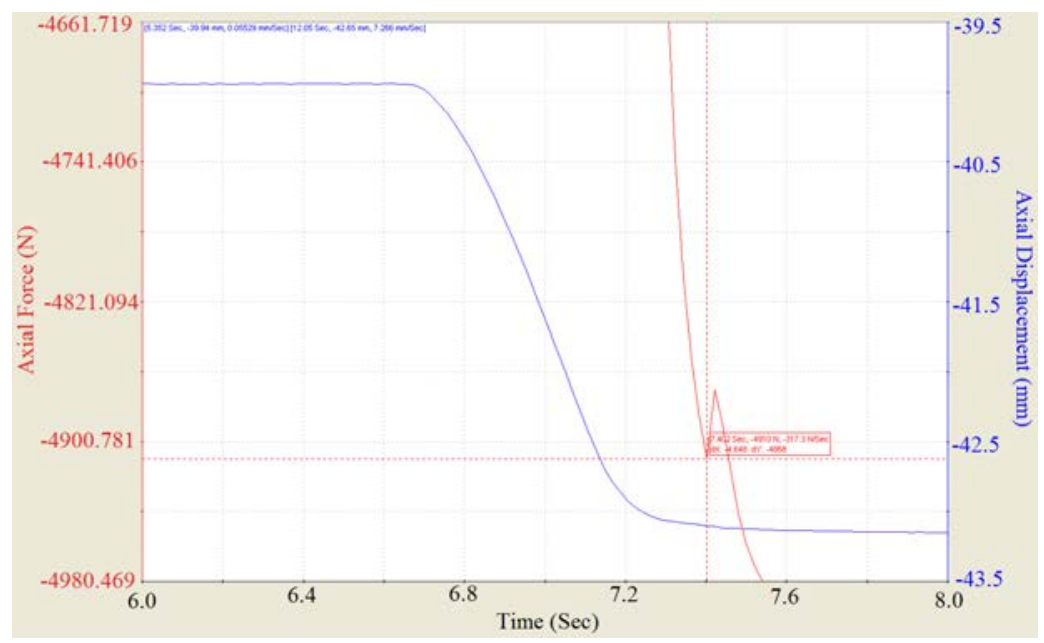

Fig. 4.28: Failure of the preloaded cymbal transducer with $0.013 "(0.330 \mathbf{m m})$ thick end caps.

After the test, the transducers were examined and it was found that they seemed to be intact including the brittle ceramic PZT disc. The perceived failures may be caused by small cracks forming within the epoxy or the PZT. Transducers loaded to the epoxy failure limit also represent a failure of the harvesting transducer even though they may still produce some power. This establishes that preloading can improve the damage resistance of transducers and is explored experimentally later.

\subsubsection{Preloading of a slitted transducer}

A new MTS ${ }^{\circledR}$ experiment was set up to determine whether preloading the transducer with six radial slits (shown in Figure 4.22) would make it more resistant to failure of the piezoceramic or bonding epoxy material. The goal of the test was to produce a higher peak voltage than the piezo unit with four radial slits while not causing a failure of the six slitted transducer shown in the bottom right corner of Figure 4.20. Figure 4.29 shows an image of the six slitted-preloaded transducer on the right, and the same transducer in its test configuration on the left. Two aluminum cylinders were machined and positioned so that the slitted cymbal transducer could be 
compressed during the test without the preloading band interfering. Two traces were gathered from testing of the slitted-preloaded transducer to check for consistency and are shown in Figure 4.30. The traces are named "Preloaded Slitted Transducer" "1" and "2" to signify test run 1 and 2. Using the same test apparatus and cycling speed as previous tests $(0.25 \mathrm{~Hz}$ and $0-400 \mathrm{~N})$, the traces were analyzed with MATLAB ${ }^{\circledR}$ to determine power dissipation and peak voltages. The resulting power calculation again used Equation 4.1 and returned power dissipation and peak voltage values values of $173.3 \mu \mathrm{W}$ with peaks of $70.4-68.8$ Volts for "Preloaded Slitted Transducer 1" trace, and $164.2 \mu \mathrm{W}$ and $76.8-75.2$ Volts for the "Preloaded Slitted Transducer 2" trace. The power dissipation results are both significantly higher than the four slit transducer and represent more than twice the power dissipation compared to the next highest power dissipation for a non-preloaded transducer. The voltage, however, is similar but below that of the four slitted non-preloaded transducer which produced $89.2 \mathrm{~V}$ at its peak, indicating that the preloading may be too high and causing detrimental results on peak voltage performance. These results should be improved due to the radial slits relieving circumferential stress within the end cap, allowing more strain energy to be absorbed by the piezo material and less to be absorbed in the end cap. By setting a preload too high, the preload band would expand and contract with the transducer, absorbing strain energy and reducing peak voltage values.
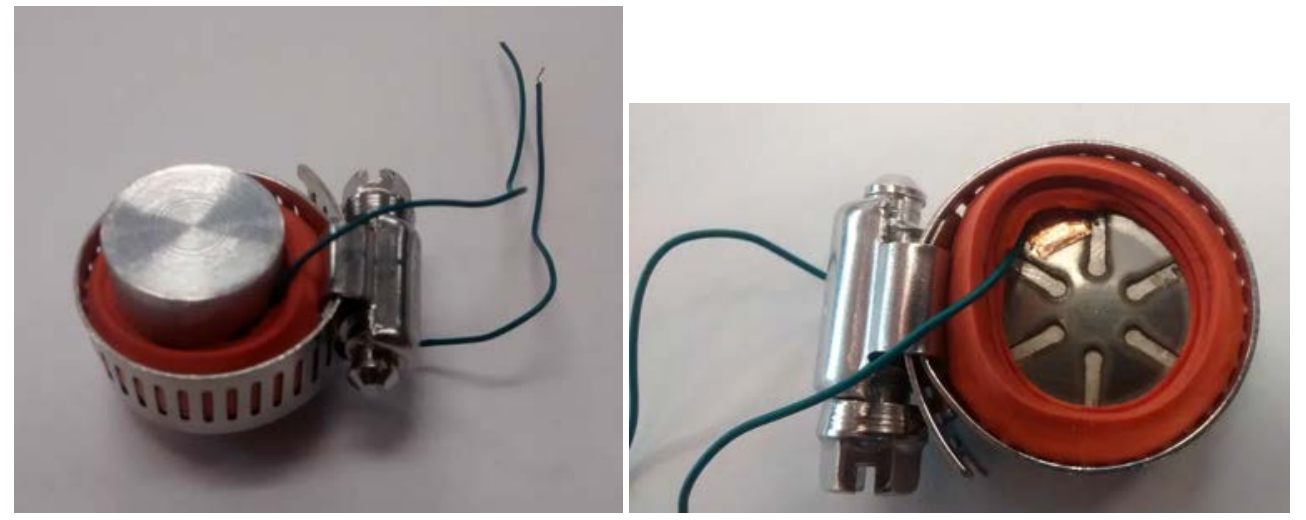

Fig. 4.29: The preloaded six slit transducer after testing (left) The same transducer with the testing pucks removed (right). 


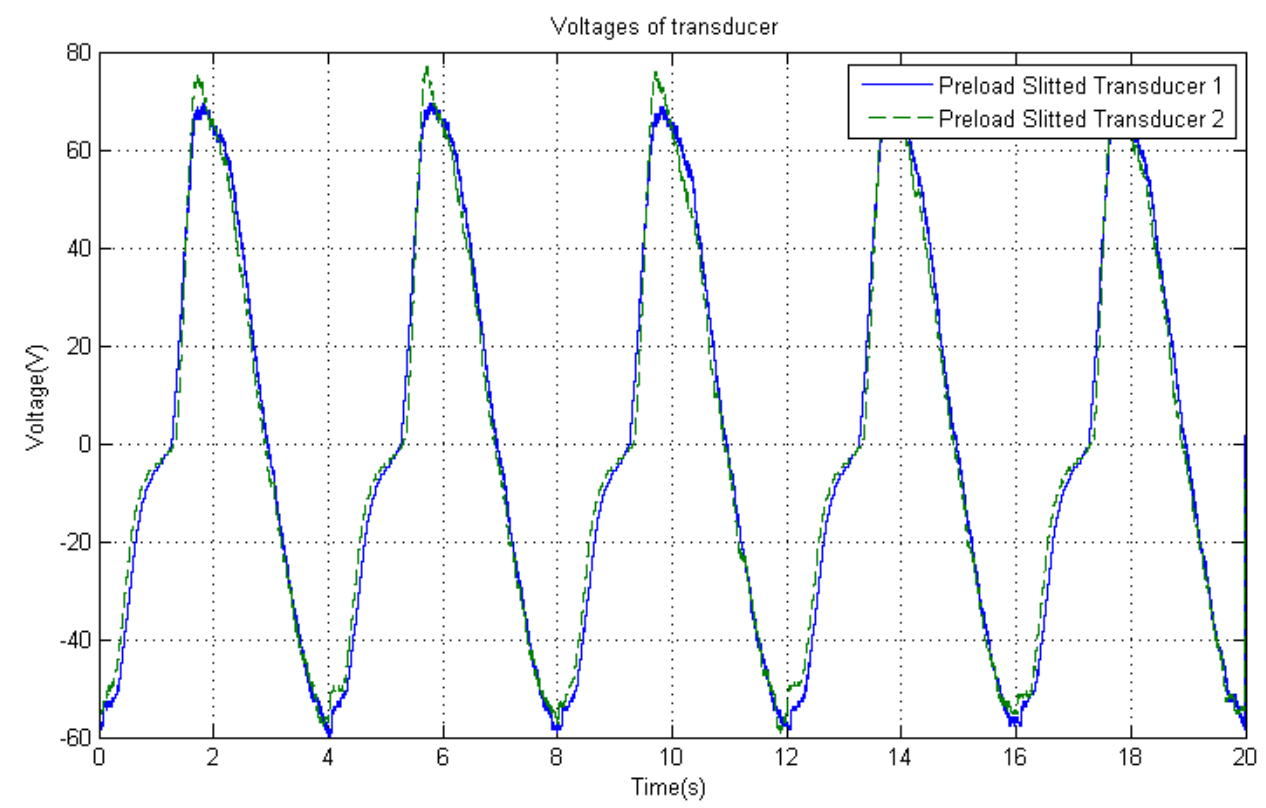

Fig. 4.30: Preloaded six slitted transducer trace.

\subsubsection{Transducer loading and end cap material experiment}

In order to properly compare a slitted and preloaded transducer to a solid end capped one, an experiment was performed to explore design refinements to the solid transducer. The purpose of this experiment was to determine weather 0.008 " 301 stainless steel would be a better performing endcap compared to the 0.01 " thick 510 phosphor bronze used on some previous solid transducers as was suggested by the end cap thickness FEA study. In addition, roughening of the end caps with sand paper was used to improve adhesion between end cap and the epoxy. Finally peak voltage and power dissipation where measured as larger forces were applied to the cymbal units to understand the role of load on the piezoelectric harvesters. The rationale behind the variations of end cap materials and thicknesses are as follows. The thickness of the stainless steel end capped transducers was chosen from the FEA end cap thickness studies performed in Chapter 3 while the thickness of the bronze transducers where chosen because of their successful use in the first roadway harvesting pad. The four 
transducers, made and shown in Figure 4.31, are numbered 1 to 4 from the left. In order to improve performance, the transducers were assembled with as little epoxy as possible to prevent ooze into the central cavity.

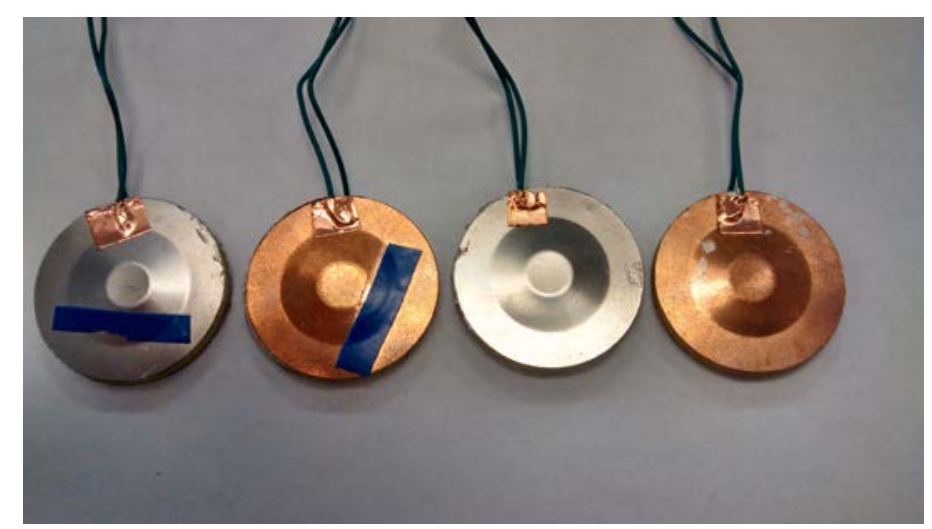

Fig. 4.31: The solid end capped transducers after the MTS ${ }^{\circledR}$ test (numbered 1-4 from the left).

Figure 4.32 shows the four transducers disassembled after the MTS ${ }^{\circledR}$ test. Transducer 1 had an end cap fall off during the test while transducer 3 had its end cap separate slightly during the test. Due to careful assembly, all transducers had smaller amounts of ooze than earlier transducers with transducer 1 having little but uniform ooze into the central cavity. Predominantly failures occurred within the epoxy and metal endcap interface, however when transducer 4 was taken apart, the epoxy separated from the PZT disc. It is noted that the epoxy for transducers 1 and 3 were tinted gray and the epoxy for transducers 2 and 4 were tinted reddish indicating that metal powder from the roughing process was left on the end caps before assembly and contributed to their failures. 


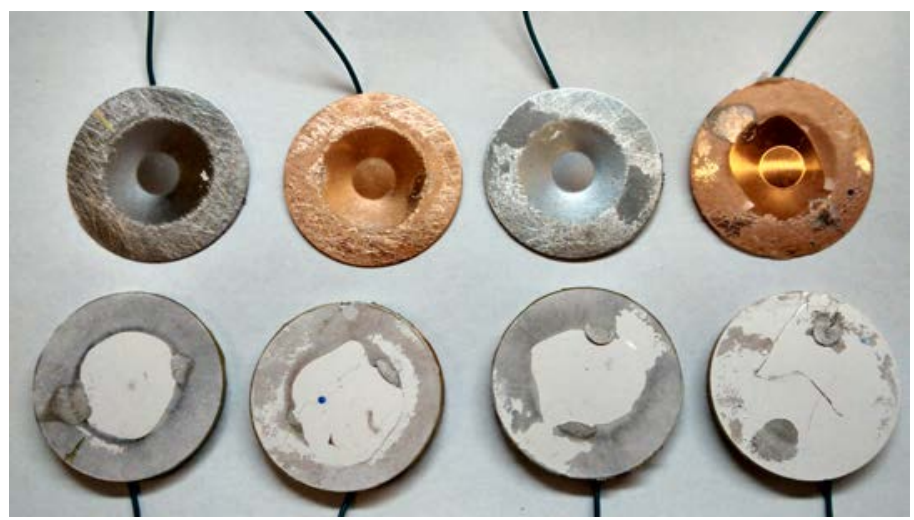

Fig. 4.32: Solid transducers after testing with end caps removed (ordered 1-4 from the left).

Table 4.5 shows the power calculated using Equation 4.1 and peak voltage for each transducer under set sinusoidal loads. The MTS ${ }^{\circledR}$ machine tended to undershoot the desired peak force level in the tests, and so the actual peak loading force is reported instead of the desired loading force. The results show that the stainless steel transducers appear to generate less power with increased load compared to the bronze endcapped transducers despite performing much better at lower force ranges. These results appear to be caused by partial or complete failure (as was the case with transducer 1 and transducer 2) within the epoxy - endcap bond. It is noted that the power dissipated and peak voltage produced by transducer 1 before failure was greater than the best performing phosphor bronze transducer at much higher loads. The highest performing tests for the phosphor bronze transducers generated power in the high 70's and low 80's $(\mu \mathrm{W})$ range and peak voltage in the low 50's range (shown in Table $4.5 \mathrm{~b}$ and $\mathrm{d}$ ), compared to the pre-failure power dissipation of transducer 1 at $96.7(\mu \mathrm{W})$ and peak voltage of $69.6 \mathrm{~V}$ (shown in Table $4.5 \mathrm{a}$ ). This output is higher than the "solid transducer" trial shown in Table 4.4 which dissipated $76.6 \mu \mathrm{W}$ and produced $57.6 \mathrm{~V}$. 
Table 4.5: Material and load MTS ${ }^{\circledR}$ test results.

(a) MTS ${ }^{\circledR}$ test results for stainless steel endcapped transducer 1.

\begin{tabular}{|c|c|c|}
\hline Transducer 1 load range $(\mathrm{N})$ & Power $(\mu \mathrm{W})$ & Peak voltage $(\mathrm{V})$ \\
\hline \hline 0-200 & 56.55 & $58.4-53.6$ \\
\hline 0-250 before failure (shown in Figure 4.33) & 96.74 & $69.6-67.2$ \\
\hline 0-250 after failure (shown in Figure 4.33) & 10.89 & $32.8-31.2$ \\
\hline
\end{tabular}

(b) MTS $®$ test results for phosphor bronze endcapped transducer 2.

\begin{tabular}{|c|c|c|}
\hline Transducer 2 load range $(\mathrm{N})$ & Power $(\mu \mathrm{W})$ & Peak voltage $(\mathrm{V})$ \\
\hline \hline $0-200$ & 28.51 & $30.8-26.4$ \\
\hline $0-225$ & 6.00 & $13.6-12.4$ \\
\hline $0-250$ & 45.11 & $38.4-36.0$ \\
\hline $0-325$ & 79.51 & $53.6-49.6$ \\
\hline
\end{tabular}

(c) MTS ${ }^{\circledR}$ test results for stainless steel endcapped transducer 3 .

\begin{tabular}{|c|c|c|}
\hline Transducer 3 load range $(\mathrm{N})$ & Power $(\mu \mathrm{W})$ & Peak voltage $(\mathrm{V})$ \\
\hline \hline $0-160$ & 28.41 & $34.4-33.2$ \\
\hline $0-250$ & 54.80 & $52.8-51.2$ \\
\hline $0-325$ & 34.48 & $52.8-51.2$ \\
\hline $0-350$ & 24.15 & $48.0-48.0$ \\
\hline
\end{tabular}

(d) MTS ${ }^{\circledR}$ test results for phosphor bronze endcapped transducer 4.

\begin{tabular}{|c|c|c|}
\hline Transducer 4 load range $(\mathrm{N})$ & Power $(\mu \mathrm{W})$ & Peak voltage $(\mathrm{V})$ \\
\hline \hline $0-160$ & 24.96 & $26.8-25.2$ \\
\hline $0-300$ & 83.81 & $51.2-48.0$ \\
\hline
\end{tabular}

The trace of the output of transducer 1 was being measured when the unit failed giving the opportunity to measure the power and voltage before and after the failure. Figure 4.33 shows the failure of transducer 1 at a sinusoidal load cycle of $0 \mathrm{~N}$ to $250 \mathrm{~N}$. This type of failure could be prevented by using a radial preload. 


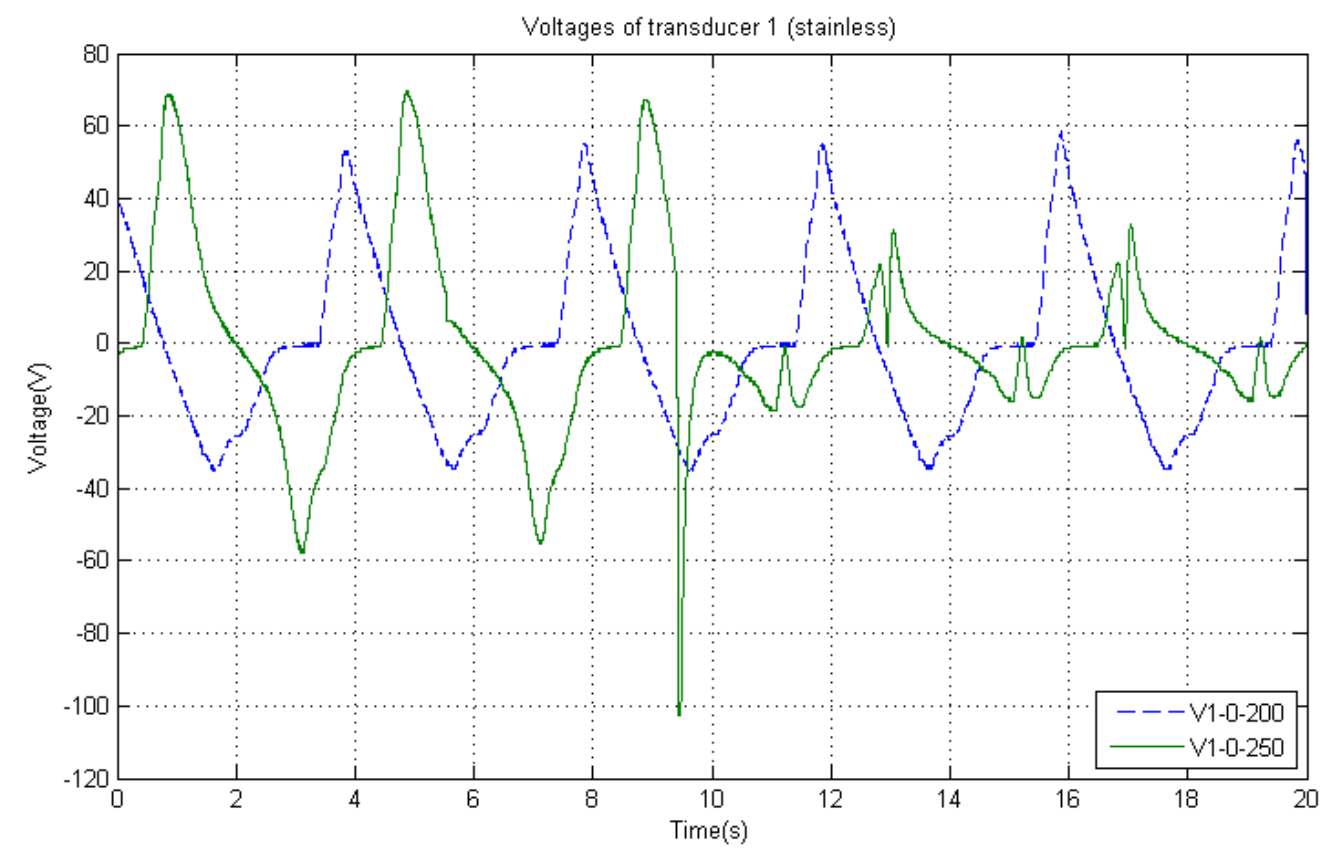

Fig. 4.33: Voltage trace of transducer 1 for a sinusoidal load of $0-200 \mathrm{~N}$ labeled "V1-0-200" and for a load range of 0 - 250 N labeled "V1-0-250".

In conclusion, due to durability, the 0.01 " thick phosphor bronze end cap will be used for comparison to the slitted transducers later in this paper where both types of transducers are built into their own harvesting pads and compared. It is the best performing cymbal transducer that is reliable enough to withstand the expected loads applied by a vehicle.

\subsection{Preload measurement}

The measurement of preload tension or preload stress is important to be able to apply the correct levels of preload. Too small or too large of a preload could cause the transducer it is supporting to fail. Three methods were attempted for the preload measurement and verification. The first method attempted utilized Fujifilm Prescale ${ }^{\top M}$ shown in Figure 4.34. This is a plastic film filled with dye capsules [51]. Under pressure the dye capsules will burst, colouring the plastic with a proportional colour to the 
peak compressive stress received. There are many different films, each with a different effective stress measurement range. The colour intensity must be compared to another sheet of film exposed to the same peak compressive stress in order to match or interpolate measurement of the stress. This method is flexible for measuring stresses in small spaces however at best, it has a $10 \%$ accuracy for stress measurements. To measure the preload stress of a transducer, the film was cut into small squares and placed in between the cymbal transducer and the elastics of the preloading system. It was found that the small pieces of prescale ${ }^{T M}$ film placed between the piezo transducer and the elastic preload bands became crumpled and this made it very difficult to get any kind of measurement from the test films. It was also found that the compressive force varied around the piezo disc, and so the prescale ${ }^{T M}$ was not deemed to be a reasonable solution.

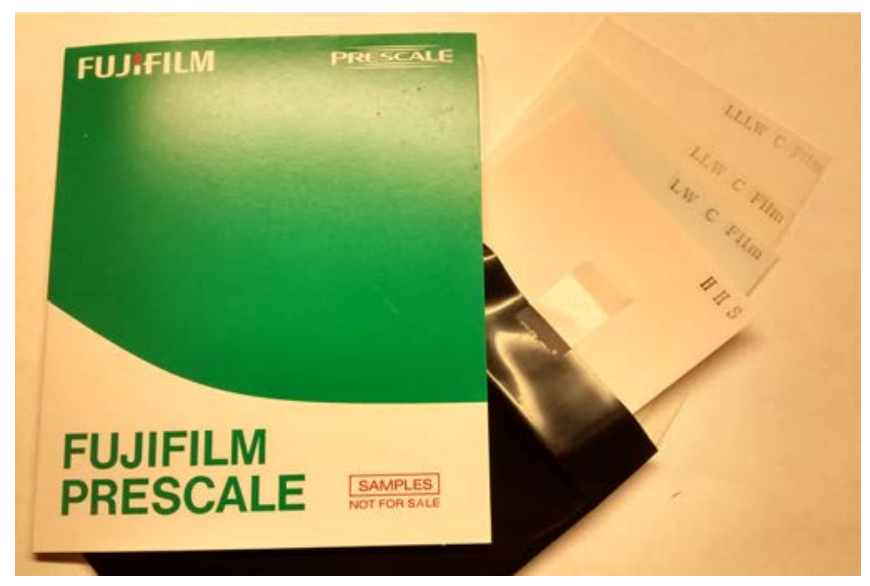

Fig. 4.34: Fujifilm Prescale ${ }^{T M}$ sample pack.

A Tekscan ${ }^{\circledR}$ FlexiForce ${ }^{\circledR}$ A201 force sensor was then attempted to be used to indirectly measure the preload stress. The sensor, shown in Figure 4.35, is far more accurate than the Fujifilm Prescale ${ }^{T M}$ however it is not meant to have its sensing pad bent. Similar to the Fujifilm Prescale ${ }^{T M}$, the sensing area experienced crumpling when placed directly between the piezo transducer and the preloading rubber bands. There was also the added danger that the crumpling would damage the sensor and prevent 
it from making accurate readings. Tekscan ${ }^{\circledR}$ recommends that no part of the sensing area be curved while taking any measurements, and so for this reason, the sensor was discarded as a preload measuring and setting sensor.

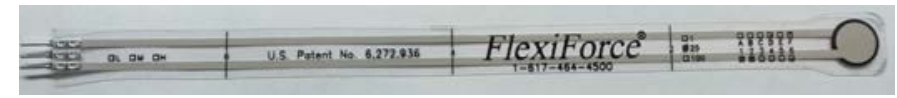

Fig. 4.35: Tekscan ${ }^{\circledR}$ FlexiForce ${ }^{\circledR}$ A201 force sensor.

Finally it was decided to directly measure the preload stress with a strain gauge on the outer surface of the pipe clamp. An Omega ${ }^{\circledR}$ KFH-03-120-C1-11L1M2R sensor (shown in Figure 4.36) was purchased for measurement of strain in the preload band. This method avoids the problems that the previous two methods had in that there is no risk of damaging the sensor from bending. The strain gauge was chosen for its small grid size $(0.3 \mathrm{~mm}$ long and $1.96 \mathrm{~mm}$ wide) so that two can be placed on either side of the worm screw slots of the pipe clamp (shown in Figure 4.37).

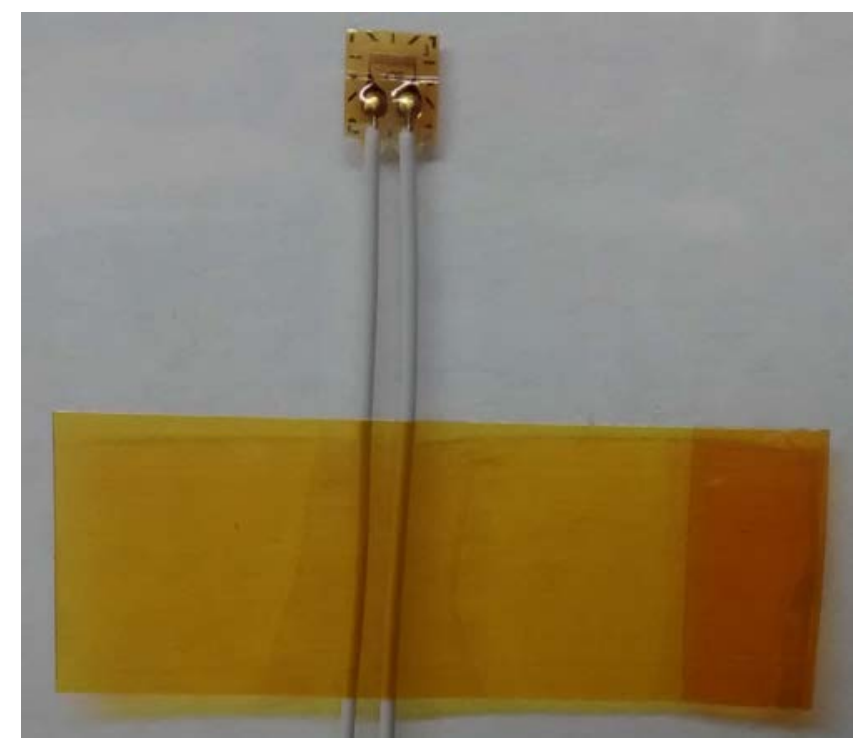

Fig. 4.36: Omega ${ }^{\circledR}$ KFH-03-120-C1-11L1M2R strain gauge. 


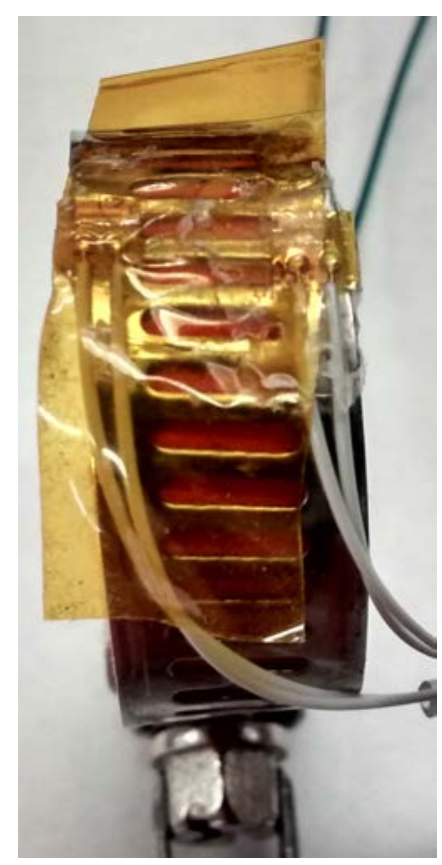

Fig. 4.37: Omega ${ }^{\circledR}$ strain gauge mounted on a preloaded transducer.

The previous model of solid transducers give an estimate of the upper bound of the preload tension of $\frac{438.41 \mathrm{~N}}{2}=219.21 \mathrm{~N} .219 .21 \mathrm{~N}$ is the preload tension which minimizes the radial stress in the PZT at a load of $367.875 \mathrm{~N}$, the estimated load experienced by an individual transducer in a harvesting pad being loaded by a $1500 \mathrm{Kg}$ vehicle. The reason that the value is divided by 2 is to reduce the radial compressive force applied by the preload tension, while allowing a tensile force to exist in the PZT during compression. It is assumed that the peak compressive force, experienced by the PZT while not loaded and the peak radial tension of the same PZT disc while fully loaded will have approximately the same magnitude. To measure the strain in the preload clamps, the cross section is needed. The areas where the strain gauges are attached are measured to be $2.72 \mathrm{~mm}$ and $2.92 \mathrm{~mm}$ wide, and $0.50 \mathrm{~mm}$ thick, and are calculated as being $(0.00272 \mathrm{~m}+0.00292 \mathrm{~m}) 0.0005 \mathrm{~m}=2.82 \times 10^{-6} \mathrm{~m}^{2}$. The average stress can be calculated as $\frac{219.21 \mathrm{~N}}{2.82 \times 10^{-6} \mathrm{~m}^{2}}=7.77 \times 10^{7} \mathrm{~Pa}$. Substituting that value into Equation 4.2 and assuming that the stainless steel band has a similar Young's modulus as 301 stainless steel, the estimated strain can be calculated as: 


$$
\begin{gathered}
Y_{x y}=\frac{x}{X} \\
X=\frac{7.77 \times 10^{7}}{1.93 \times 10^{11}}=4.03 \times 10^{-4}
\end{gathered}
$$

The strain resistance relation provided by Omega ${ }^{\circledR}$ is shown in Equation 4.3 and is used to calculate the change in resistance expected at the target preload stress using a gauge resistance of $120.4 \Omega$, and a gauge factor, $G F$, of 1.51:

$$
\begin{gathered}
X=\left(\frac{\Delta R_{g}}{R_{g}}\right) / G F \\
\Delta R_{g}=1.51 \cdot 4.03 \times 10^{-4} \cdot 120.4 \Omega=0.0732 \Omega .
\end{gathered}
$$

where $R_{g}$ is the gauge resistance. Because of the peak rated voltage of the strain gauge being $1.5 \mathrm{~V}$, a voltage of $3 \mathrm{~V}$ was used for the Wheatstone bridge (shown in Figure 4.38) to make measurements.

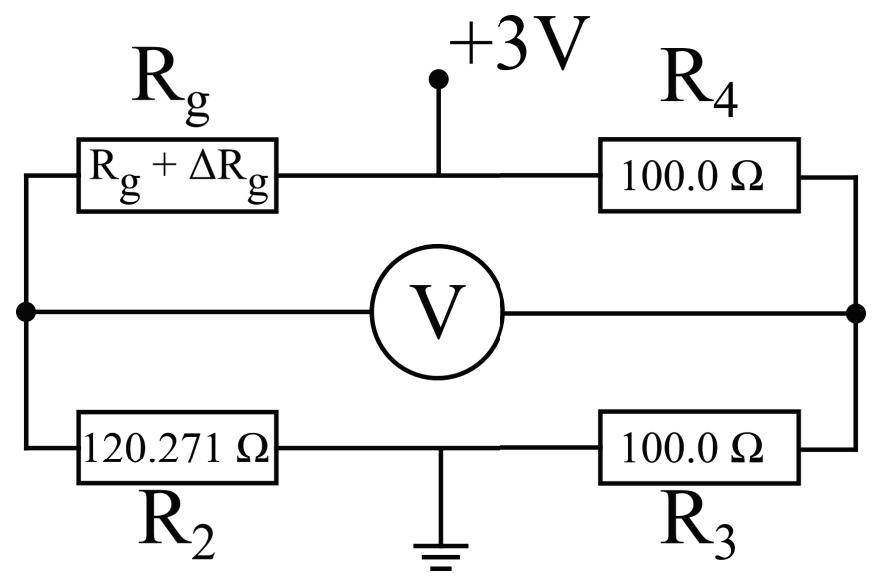

Fig. 4.38: Bridge circuit used to measure preload stress. 
Standard measurements using a Wheatstone bridge will replace on of the resistors in the bridge with a potentiometer. The reason for this is to use the potentiometer to ajust the circuit such that the measured output voltage is zero. This compensates for small amounts of current leakage from the measurement device. Because of electrical noise introduced to the measurements by the potentiometer used, it was left out and the Wheatstone bridge was left calibrated. Instead the voltage across the center was measured, and is calculated as:

$$
\begin{gathered}
V_{\text {out }}=V_{\text {in }}\left[\frac{R_{g}+\Delta R_{g}}{R_{g}+\Delta R_{g}+R_{2}}-\frac{R_{4}}{R_{3}+R_{4}}\right] \\
V_{\text {out }}=3\left[\frac{120.4732}{120.4732+120.271}-\frac{100.0}{100.0+100.0}\right]=1.30 \mathrm{mV} .
\end{gathered}
$$

A Tektronix ${ }^{\circledR}$ DMM4050 digital multimeter was used to make voltage measurements using the Wheatstone bridge. A Tektronix ${ }^{\circledR}$ PWS4305 power supply was used to provide the 3 volts needed to the bridge circuit. Because the initial voltage across the bridge would not be exactly zero because the bridge wasn't compensated for, the preload was adjusted until the output voltage was equal to the sum of the initial measured voltage before preloading and the calculated voltage in Equation 4.4. Figure 4.39 shows a transducer set to the desired preload value with two strain gauges attached for strain verification. 


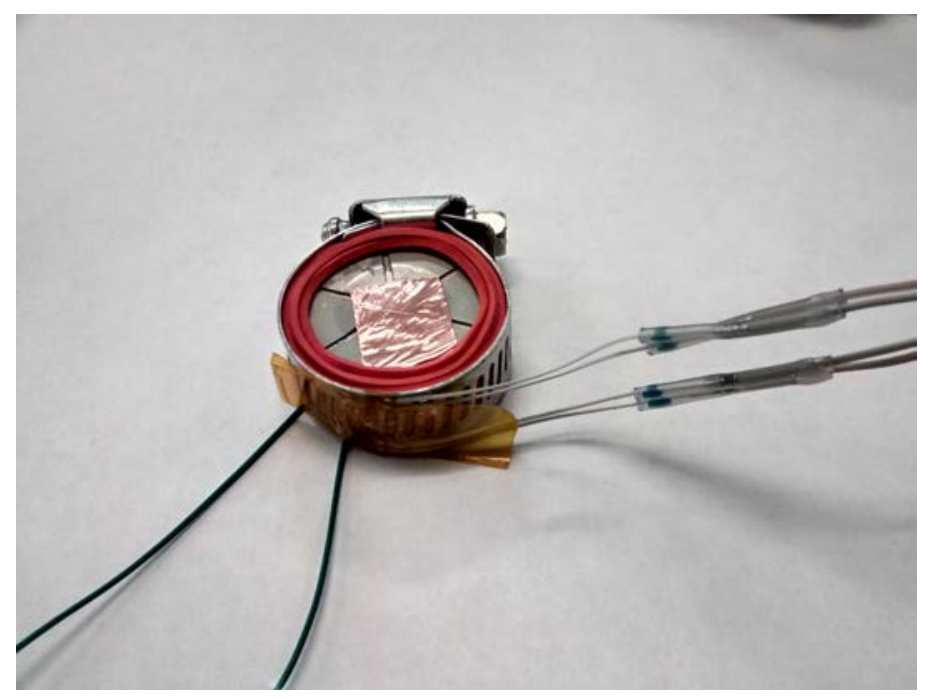

Fig. 4.39: A preloaded and slitted transducer set to the correct preload.

\subsection{Harvester design}

The purpose of a harvester is to house the transducers and protect them during testing involving heavy vehicles. The overall design places transducer pairs into a machined and cast nylon containers which are then implanted into commercial rubber speed bumps. An aluminum cover panel is then used to protect the transducers from the vehicle tires and to distribute the vehicle load across all the transducers.

\subsubsection{Speed bump}

A Barco Mini Speed Hump, shown in Figure 4.40, was purchased for modification to become a piezoelectric power harvesting speed hump technology demonstrator. This was chosen because of the solid rubber construction designed to be installed on a roadway, the small modular size, and the relatively low cost. In addition, the speed hump has yellow sections which are convenient for removal and replacement with power harvesting modules. The two sections nearest the middle of the hump range 
from $26 \mathrm{~mm}$ to $32 \mathrm{~mm}$ in thickness, sufficient to allow room for the construction of cast nylon transducer containers.

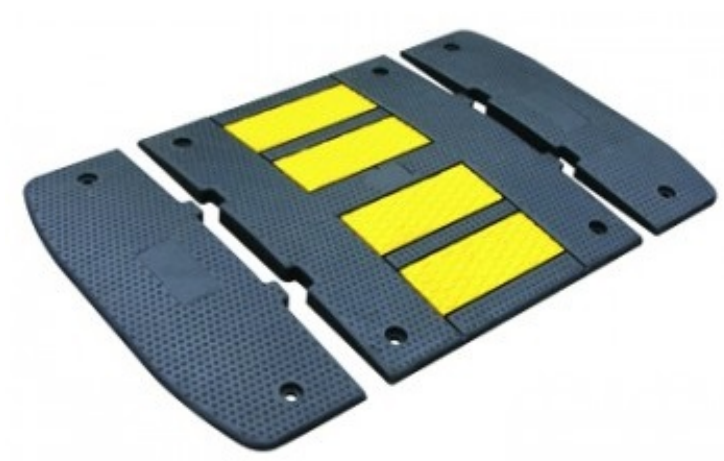

Fig. 4.40: Barco mini speed hump section.

\subsubsection{Power harvesting module}

The purpose for the power harvesting modules is to behave as a container to protect the transducers it is housing from precipitation, dirt, and damage while acting as a hard surface to press against the transducers. The housing is designed to accommodate transducers in vertical stacks, two transducers high to improve power generation. Ten pockets are arranged in two rows of five in order to accommodate ten transducer pairs, or twenty individual transducers per harvesting module. Two types of power harvesting modules were manufactured in order to house solid end capped transducers without preloading and the novel slitted and preloaded transducers. The two sets of power harvesting units to be compared are housed in the specialized modules shown in Figures 4.41 and 4.42 , the former of which is designed for preloaded transducers, and the latter for the conventional units. Cast nylon was used and purchased from McMaster-Carr ${ }^{\circledR}[48]$ due to its low cost, ease of machining, resistance to water corrosion, rigidity, and dielectric properties. 


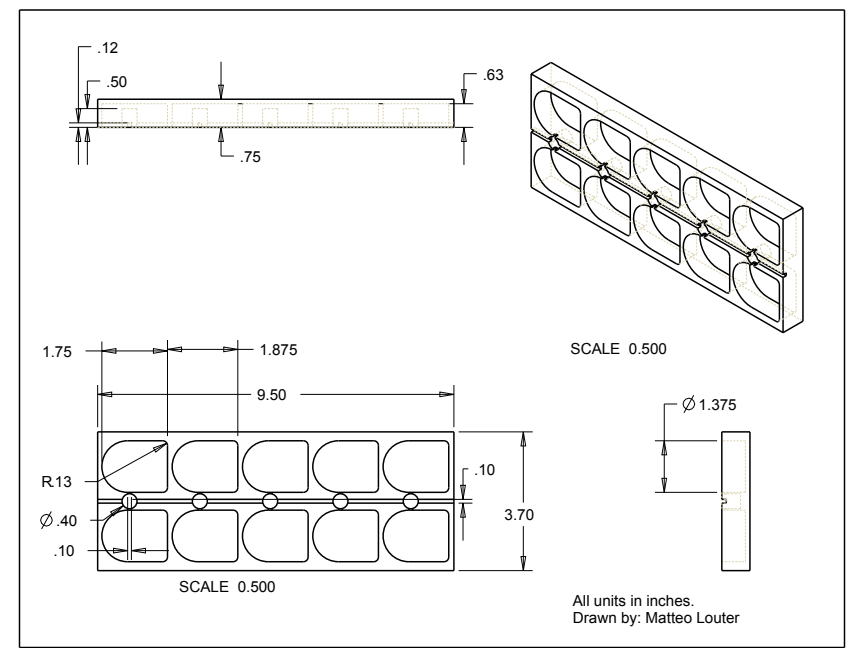

Fig. 4.41: Power harvesting module design for accommodating preloaded transducers.

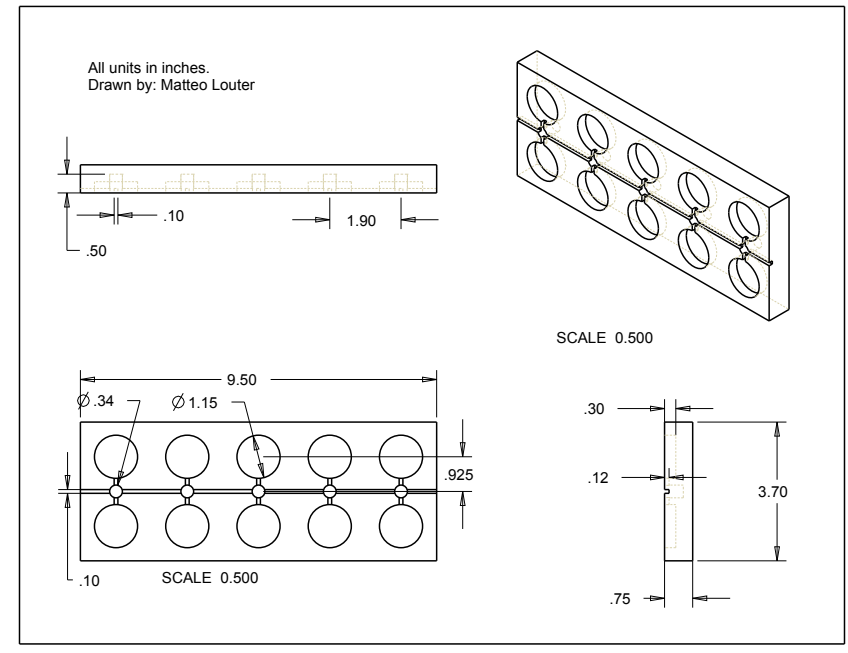

Fig. 4.42: Power harvesting module design for standard cymbal transducers.

\subsubsection{Manufacturing of preloaded transducers}

The preloaded transducer end caps use a six slitted design cut using a water jet cutter from 301 stainless steel. The two end caps in the bottom half of Figure 4.43 are shown after water jet cutting and deburring is complete. The end caps in the top half of Figure 4.43 are the result of punching the water cut discs into their final shape using the stamping setup shown in Figures 4.2 and 4.3. The slit FEA model determined 
that 0.015 " thick end cap is a suitable thickness, while the non slitted end caps used the same 0.01 " thick phosphor bronze as the earlier, successfully tested, transducers did.

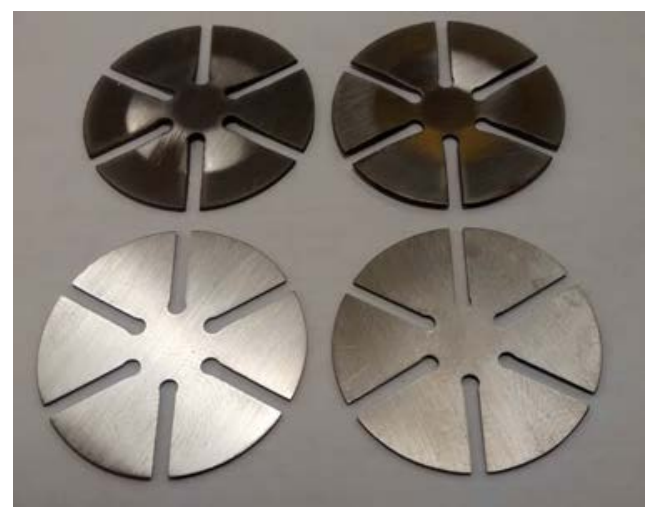

Fig. 4.43: Slitted end caps before and after stamping.

After the slitted end caps are punched into the desired cymbal shape, they are roughened and cleaned to better adhere to the LePage ${ }^{\circledR}$ speed set $^{\mathrm{TM}}$ professional epoxy and to the PZT discs. Figure 4.44 shows the transducers during the curing process held together with six binder clips (left) and at the end of the process with the binder clips off (right). In addition to the conventional epoxy, small dots of MG Chemicals ${ }^{\circledR}$ silver conductive epoxy were used in order to conduct the power harvested to the end cap material where the wiring will be attached.
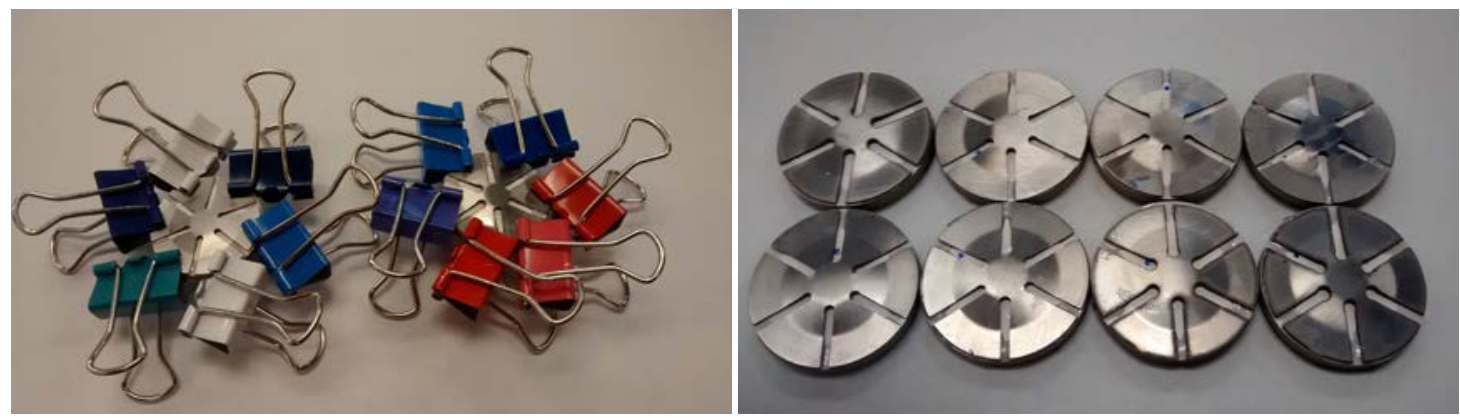

Fig. 4.44: Preloaded transducers being epoxied (left) and the hardened transducers (right).

In order to improve power generation, transducers were paired and stacked to increase 
the compression displacement and double the power generated for each transducer stack in the housing. Figure 4.45 shows the transducer pairs electrically connected to each other along with wiring (left) using electrically conductive tape and shows the completed transducers with rubber buffers and preloading clamps in the right image. Transducers were paired such that their polled directions face each other. This allowed the contact point between transducers to be conductive and generate a positive potential when the pair is compressed on the top of the upper transducer. The top of the upper transducer and bottom of the lower transducer are then connected with conductive tape and represent the negative terminal of the transducer pair. The preloading utilized three thick lobster claw elastic bands to reduce the stress concentrations from the irregular pressure of the steel pipe clamp screw mechanism being pressed against the PZT ceramic disc.
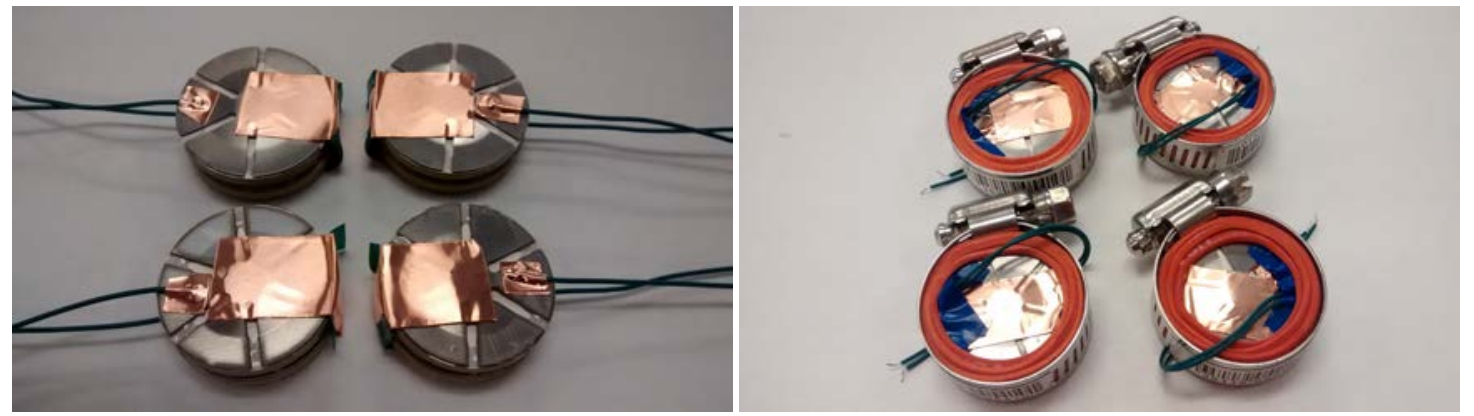

Fig. 4.45: Preloaded transducers being paired up and electrically connected (left) and the finished preloaded transducers (right).

The completed preloaded transducers are then placed inside their machined nylon housing (shown in Figure 4.46). Each transducer pair is then wired to their own DF04M Fairchild ${ }^{T M}$ Semiconductor full wave bridge rectifier rated for $400 \mathrm{~V}$ and $1.5 \mathrm{~A}$ to handle high voltage and current spikes. 


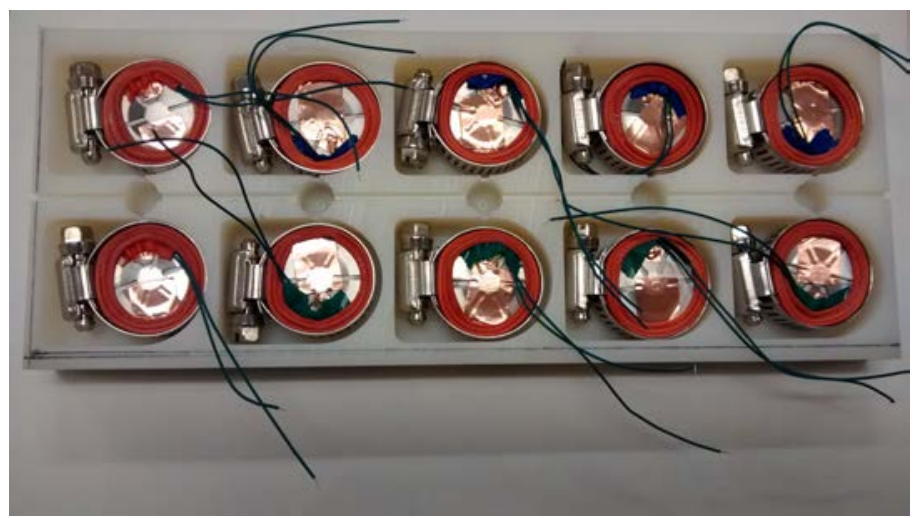

Fig. 4.46: Preloaded transducer housing after machining with inserted transducers.

Figure 4.47 depicts a malfunctioning transducer with its aluminum spacers to help it contact the container cover and container bottom so load it applied in the correct location. The defect was caused by conductive tape used to electrically connect the negative terminals of the transducer pair being too short for some harvesting transducer pairs and prevented the transducers from remaining horizontal and parallel when being compressed. Care was taken to prevent such manufacturing defects. Finally $3.2 \mathrm{~mm}$ thick by $12.6 \mathrm{~mm}$ diameter aluminum discs (also shown in Figure 4.47) are machined to increase the thickness of the two transducers such that their thickness is greater than the width of the pipe clamp. This is necessary to conduct the vehicle load from the top cover, through the transducer pairs, to the ground. 


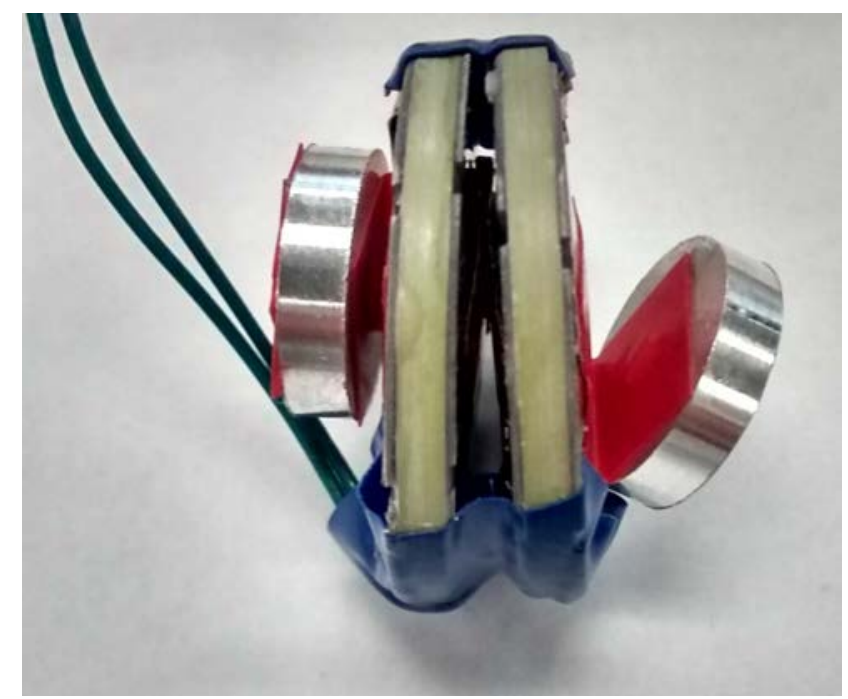

Fig. 4.47: Under performing slitted transducer.

\subsubsection{Solid transducers}

It was primarily damage resistance that the 0.01 " thick phosphor bronze end caps were chosen over the 0.008 " thick stainless steel end caps. The solid transducer nylon housing was machined and is shown installed in the rubber speed bump in Figure 4.48. Also shown is the 0.25 " thick aluminum cover plate to more evenly distribute a vehicle load over the transducers as well as prevent the transducers from wear and dirt from the vehicle tire. Figure 4.49 shows the solid transducers assembled and installed in the housing unit. Similar to the preloaded transducers, solid transducers are stacked in pairs, and each pair connected to its own voltage rectifier. Electrical tape is used to hold the wiring in channels machined in the nylon. 


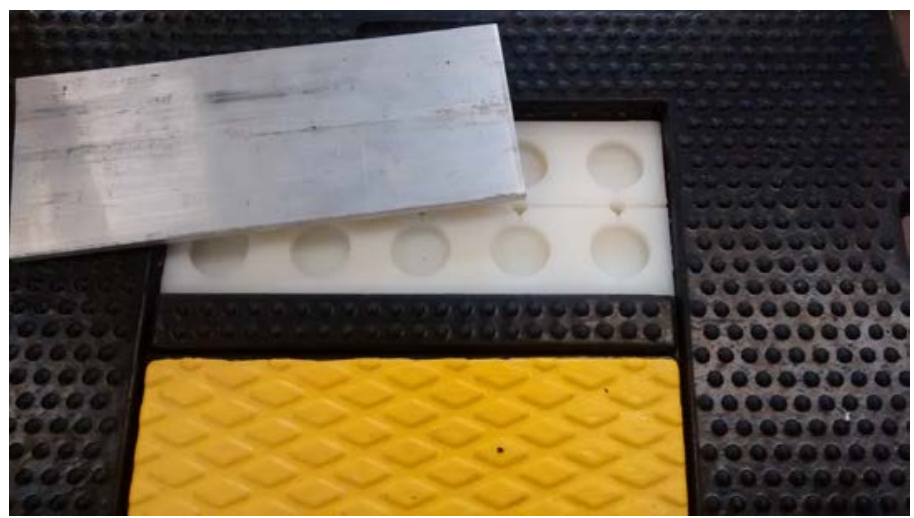

Fig. 4.48: Machined non preloaded transducer housing module sitting in the speed bump.

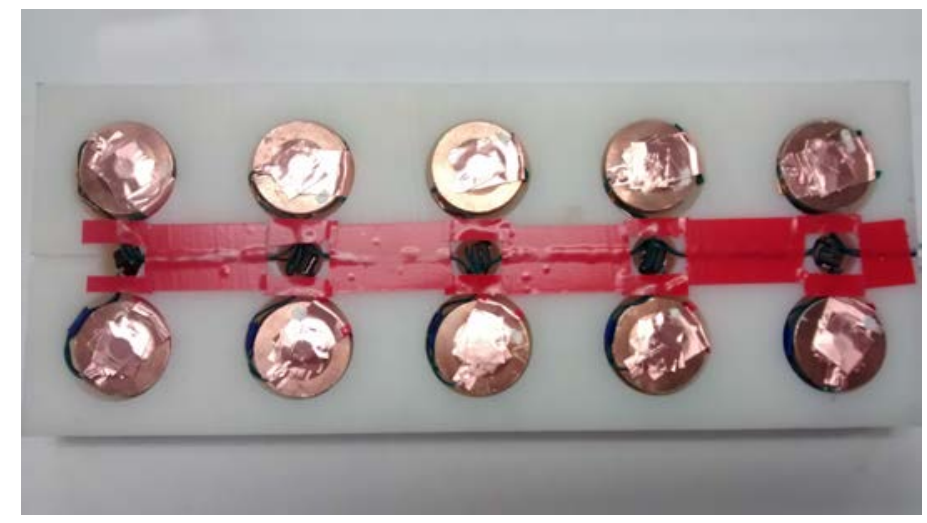

Fig. 4.49: Fully assembled non preloaded harvester.

The Cymbal transducer end caps where roughened and cleaned to improve adhesion and remove bronze powder that would interfere with the end cap epoxy interface.

\subsubsection{Assembly of the harvesting speed bump}

The two completed harvesting modules were inserted into the speed bump pockets made by removing the two central yellow rubber sections (shown in Figure 4.50). Holes bored in the side of the speed bump act as external cable routing for the harvesting modules. The transducer pairs were each attached to their own respective DF04M Fairchild ${ }^{T M}$ Semiconductor bridge rectifier rated for $400 \mathrm{~V}$ and $1.5 \mathrm{~A}$, all connected to a common pair of power bus lines shown leaving the speed hump on the 
left side of Figure 4.50. The rectifiers are chosen for their low cost, high voltage tolerances, and DIP package for ease of soldering. Finally, $0.25 "$ thick aluminum cover plates are placed on top of the harvesters, shown in the right image of Figure 4.50, for protection and even loading of the transducer pairs.
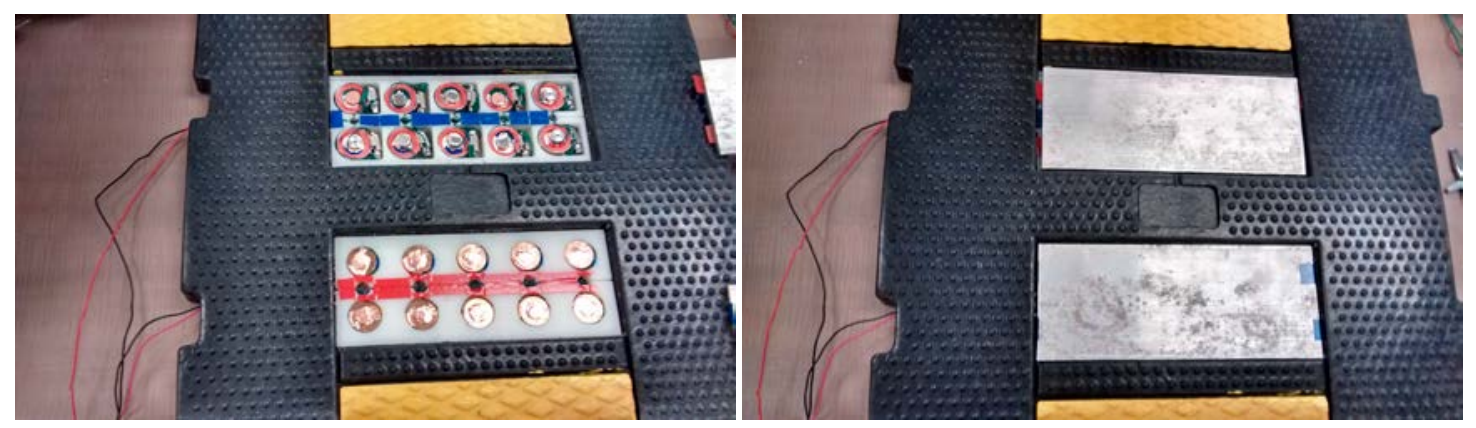

Fig. 4.50: Harvesters inserted into speed bump.

\subsection{Vehicle testing}

In order to verify the applicability of the roadway power harvesting system, practical tests are required. Though some tests can be carried out on the hydraulic test machine, many factors cannot be reproduced on it such as non centered loading and the sharp compression from a vehicle moving at high speed. The test vehicles weight was determined by using a gravel pit scale, used for weighing dump trucks and was measured as $1,800 \mathrm{~kg}$. This type of vehicle scale has a low resolution of $\pm 100 \mathrm{~kg}$ and can not measure individual wheels or axles.

\subsubsection{Initial vehicle test setup}

The initial roadway power harvester was tested using a vehicle in a parking garage as shown in Figure 4.51. The purpose of the location was to have access to a power outlet to operate the Tektronix ${ }^{\circledR}$ DPO 2024B oscilloscope that was used for all testing experiments as well as providing an environment free of precipitation and other harsh 
weather conditions. A 2003 Subaru outback, station wagon with a total measured vehicle mass of $1,800 \mathrm{~kg}$, with fuel and driver, was driven over the speed bump multiple times using the heavier front and lighter rear tire to examine power generation using differing vehicle loads. The bump was oriented such that the solid transducer harvesting pad would be compressed first as the car traversed over the speed bump in its forward direction, and the preloaded units would be compressed after. Tests were performed to compare vehicle driving direction to see what effect the direction of a traversing vehicle may have on the voltages generated. The vehicle was also driven at various speeds to determine if and how much, speed correlated with power generation. Finally the two harvesting pads were connected together to examine the voltage profile that a harvesting circuit would experience. The test setup shown in Figure 4.51 involved the power modules being directly connected to the oscilloscope which was connected to a wall outlet using an extension cord. The oscilloscope was connected to a laptop to collect the raw data and to perform data analysis later on. Other tests were performed, examining the performance of potential harvesting circuits, however that work is outside the scope of this thesis and is not discussed here.

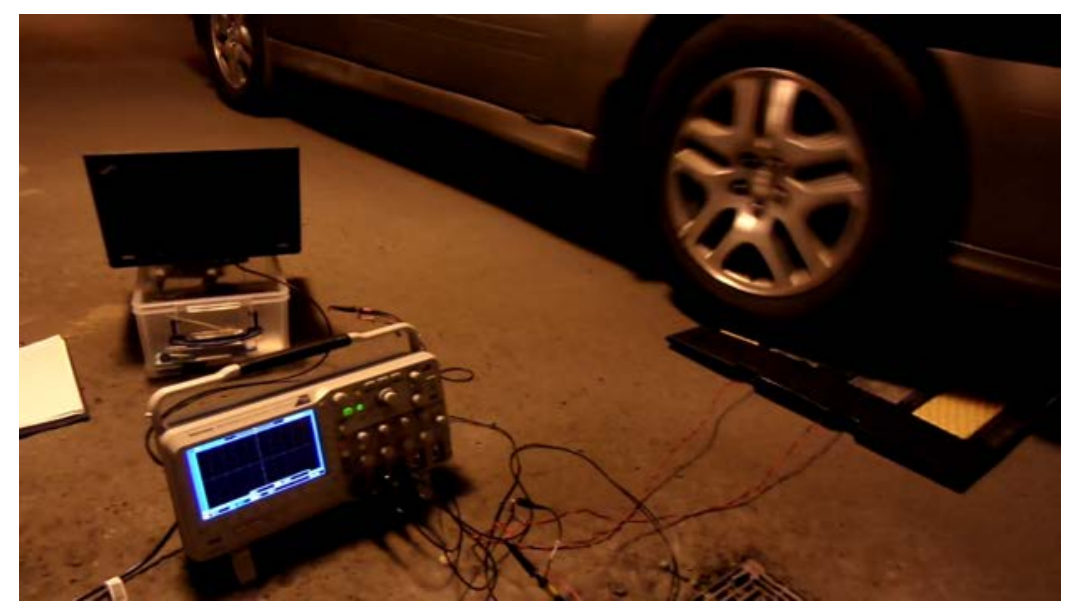

Fig. 4.51: Vehicle test setup. 


\subsubsection{Test results}

The initial vehicle tests were composed of seven discrete trials. The first simply recorded the output from the two harvester pads, the pads where traversed in two directions to examine any differences, and the third trial connected the harvesting pads together to see what effect that would have if any on the output. The fourth test through the sixth test examined various characteristics of a harvesting circuit and finally the seventh test compared power generation while driving at faster and slower speeds. Energy dissipation per wheel was calculated by using the Equation 4.1. Alternatively, the total energy harvested by the circuit model used in Section 2.2 would be the sum of the energies stored in the voltage peaks:

$$
E=\sum_{\text {peaks }} \frac{1}{2} \mathrm{C} V^{2}
$$

where $C$ is the total capacitance of all the PZT discs in all the transducers combined. It was noticed that comparing the initial trace rise times to each other, and considering the spacing of $162 \mathrm{~mm}$ between the power harvesting pads, the vehicle speed could be determined. The rise time used was determined by the times of the first instance of the voltage trace exceeding $50 \mathrm{~V}$ and was calculated using Equation 4.6.

$$
v=\frac{0.162 \mathrm{~m}}{\Delta \mathrm{t}}
$$

where $\Delta \mathrm{t}$ is the time difference between the rise times of the two harvesters traces. The speed was calculated and is shown along with the initial vehicle testing results 
shown in Table 4.6. The energy values in the column denoted "Energy dissipated per tire compression" are calculated using Equation 4.1 and represent energy dissipated through the oscilloscope. Test trials were conducted a single time making uncertainty analysis difficult, however the uncertainty from the values in these tests can be estimated from the uncertainty values measured and shown in Tables 4.1, 4.3, 4.4, and 4.5. It was possible to get an uncertainty estimation from trial 3 because several compressions of the harvesting pads were done. In order to calculate the speed, the traces of the separate harvesting pads must be kept separate. It is for this reason that a vehicle speed could not be estimated for trial 3, as the inputs of the two harvesting pads were combined.

Table 4.6: Initial vehicle test results.

\begin{tabular}{|c|c|c|c|c|}
\hline Trial & $\begin{array}{c}\text { Average } \\
\text { speed } \\
\text { between } \\
\text { harvesting } \\
\text { pads }\end{array}$ & Harvester pad & $\begin{array}{c}\text { Energy } \\
\text { dissipated per } \\
\text { tire compression }\end{array}$ & $\begin{array}{c}\text { Peak voltage } \\
\text { measured }\end{array}$ \\
\hline \multirow{2}{*}{1} & \multirow{2}{*}{$2.19 \frac{\mathrm{Km}}{\mathrm{h}}$} & Solid & $1.59 \mathrm{~mJ}$ & $\bar{~} 316 \mathrm{~V}$ \\
\hline & & $\begin{array}{l}\text { Slitted and } \\
\text { preloaded }\end{array}$ & $0.775 \mathrm{~mJ}$ & $302 \mathrm{~V}$ \\
\hline \multirow{2}{*}{2 backward } & \multirow{2}{*}{$3.34 \frac{\mathrm{Km}}{\mathrm{h}}$} & Solid & $1.19 \mathrm{~mJ}$ & $298 \mathrm{~V}$ \\
\hline & & $\begin{array}{l}\text { Slitted and } \\
\text { preloaded }\end{array}$ & $0.572 \mathrm{~mJ}$ & $256 \mathrm{~V}$ \\
\hline \multirow{2}{*}{2 forward } & \multirow{2}{*}{$3.04 \frac{\mathrm{Km}}{\mathrm{h}}$} & Solid & $1.18 \mathrm{~mJ}$ & $310 \mathrm{~V}$ \\
\hline & & $\begin{array}{l}\text { Slitted and } \\
\text { preloaded }\end{array}$ & $0.626 \mathrm{~mJ}$ & $278 \mathrm{~V}$ \\
\hline 3 & - & Combined & $1.58 \mathrm{~mJ}$ & $306-296 \mathrm{~V}$ \\
\hline \multirow{2}{*}{7 slow } & \multirow{2}{*}{$1.30 \frac{\mathrm{Km}}{\mathrm{h}}$} & Solid & $2.07 \mathrm{~mJ}$ & $288 \mathrm{~V}$ \\
\hline & & $\begin{array}{l}\text { Slitted and } \\
\text { preloaded }\end{array}$ & $1.43 \mathrm{~mJ}$ & $304 \mathrm{~V}$ \\
\hline \multirow{2}{*}{7 fast } & \multirow{2}{*}{$13.16 \frac{\mathrm{Km}}{\mathrm{h}}$} & Solid & $0.223 \mathrm{~mJ}$ & $344 \mathrm{~V}$ \\
\hline & & $\begin{array}{l}\text { Slitted and } \\
\text { preloaded }\end{array}$ & $0.286 \mathrm{~mJ}$ & $450 \mathrm{~V}$ \\
\hline
\end{tabular}

The traces from the first experiments shown in Figure 4.52 and Figure 4.53 depict the raw voltage trace from both preloaded-slitted and solid transducers. The solid 
transducers produced $29 \mathrm{~V}$ higher peak voltages on average for the first two trials. This is likely caused by incorrect preloading or manufacturing inconsistencies. In Figure 4.52, the peak voltages of both harvesting pads reach just over $300 \mathrm{~V}$. This is a rough standard for what each harvesting pad is capable of outputting.

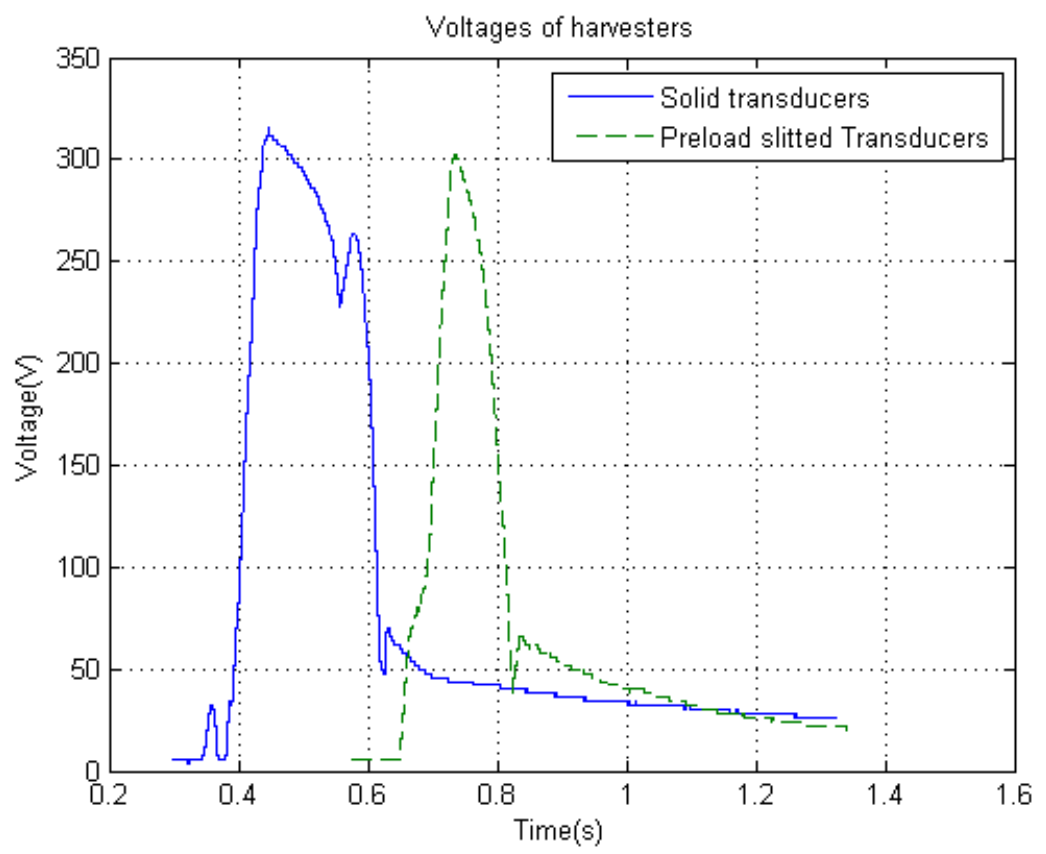

Fig. 4.52: Trial 1 traces.

It was thought that one possible cause of peak voltage variation between the harvesting pads could be the direction of travel over the speed bump. The speed bump was placed on the road such that the solid transducer pad would be compressed just before the slitted and preloaded pad. As the vehicle drove forward, it would experience a slight upward acceleration as it went over the the speed hump. This action would cause more force to be applied on the front side of the bump. Comparison of the graphs in Figure 4.53 and comparison of the energy dissipated from the two parts of the second trial, shown in Table 4.6 indicates that this difference between "forward" and "backward" driving directions does not seem to significantly affect the peak voltage results of $298-310 \mathrm{~V}$ for the Cymbal transducer, and $256-278 \mathrm{~V}$ for 
the preloaded and slitted transducer. It is also noticed in Figure 4.53 that a secondary voltage peak exists for the solid transducers. Because this peak is reversed with the reversal of driving directions, it is likely caused by a structural component such as the cover plate tilting while the vehicle load is applied across the covers surface.
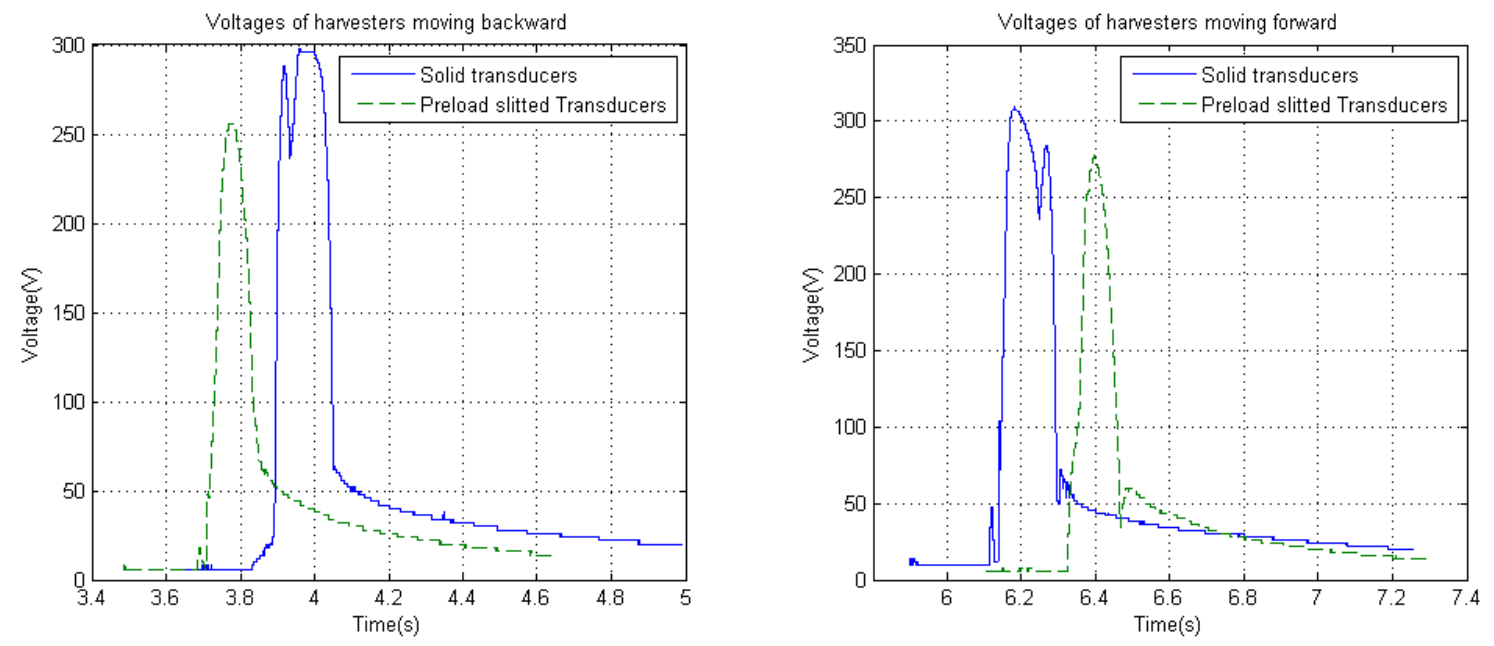

Fig. 4.53: Trial 2 traces showing backward driving on the left, and forward driving on the right.

The third trial attempted to understand the effects of combining multiple harvesting pads together to see if they would have a detrimental effect on each other or peak voltage generation. The results for trial 3 shown in Figure 4.54 and numerically in Table 4.6, show that the energy dissipated per tire compression, along with the peak voltage, from trial 3 remained similar to the other tests at $306-296 \mathrm{~V}$. The average peak voltage of the other trials excluding trial 7 is 293.3 Vso the combined pad voltage result is the result expected if they did not have an effect on one another and represent a doubling of potential power to a harvesting circuit as per Equation 4.5. The disadvantage of connecting the terminals of both power harvesting pads, is that speed determination is now more difficult to estimate and prone to more errors. 


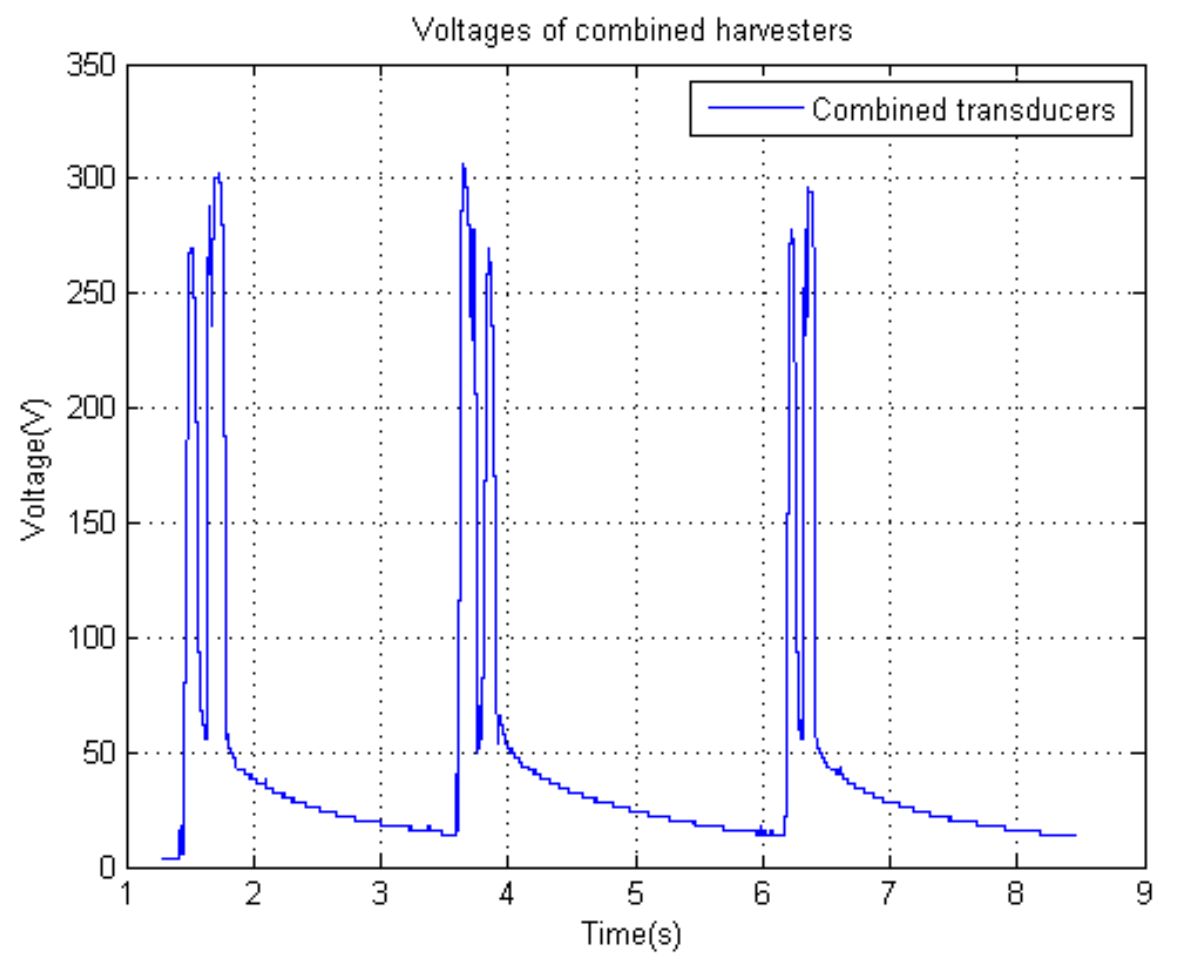

Fig. 4.54: Trial 3 traces.

Finally trial 7 tested to see if any differences existed with the vehicle velocity, which would cause the pulse width to shorten with higher velocities. The result from trial 7 appears to indicate that the peak output voltages for the slitted and preloaded transducer are highly dependent on the traversing vehicle speed with the slitted and preloaded transducer reaching the maximum measurement value of the oscilloscope at $450 \mathrm{~V}$. This apparent strong dependence on speed was unexpected and so further exploration into this was performed. Figure 4.55 depicts the slitted and preloaded transducer traces producing significantly higher peak voltages than the solid transducers at a higher vehicle speed. This is potentially caused by larger forces and deformations within the transducers, causing the more flexible slitted transducers to deform its PZT material further than the solid transducers are able to. The calculated speed is shown in the title of each graph in Figure 4.55 and is calculated by dividing the spacing of the transducer pads by the difference in measured rise times. 

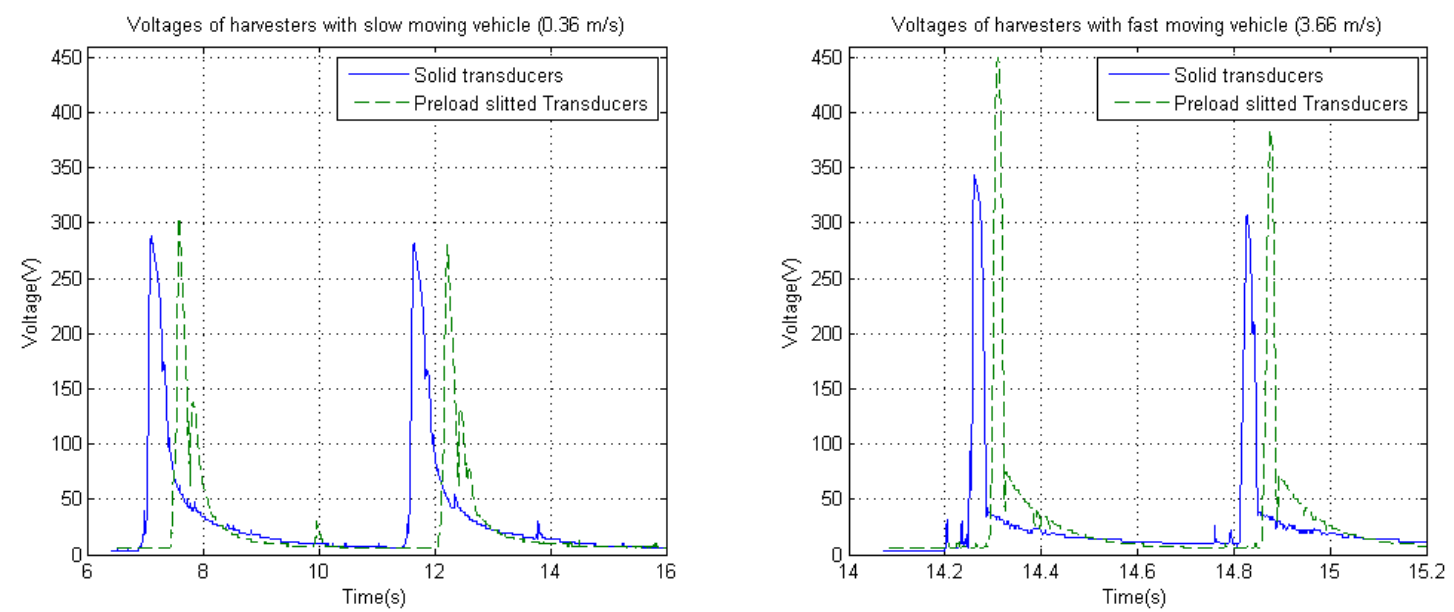

Fig. 4.55: Trial 7 traces showing voltage spikes corresponding to the heavier front, and lighter rear tire loads.

Plotting all of the peak voltages for the tests, shown in Table 4.6, with their calculated speed produces Figure 4.56. The slower speed experiments do not exhibit a correlation with speed and peak voltage. The single higher speed test implies that, especially for the preloaded and slitted transducer, that an increased speed will improve harvester performance so this anomaly is explored in the following vehicle test.

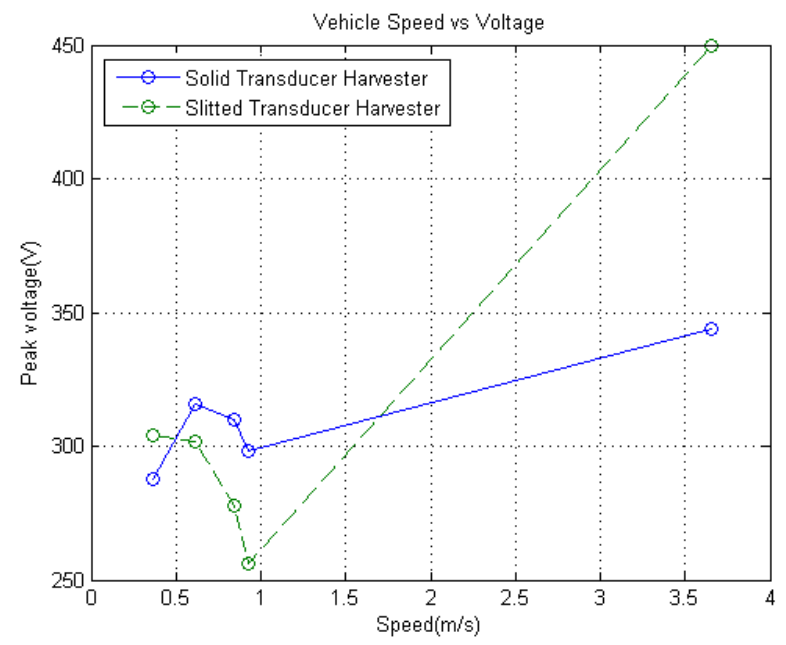

Fig. 4.56: Summary of all the peak voltages of the August vehicle testing. 


\subsubsection{Effect of vehicle speed on power generation}

In order to further explore the effect of vehicle speed on peak voltage and power generation, another test was performed to explore this relation. In order to improve the consistency of compressions applied to adjacent transducer pairs within a harvesting pad, small foam pads were applied to the tops of the transducers for one of the trials. It is possible that minute differences in thickness of the transducer pairs could cause uneven transducer loading, and so foam pads would compensate for any height differences between transducers. The experiments took place in an outdoor parking lot, using a computer backup battery to power the same Tektronix ${ }^{\circledR}$ DPO 2024B oscilloscope as a wall outlet was not available. The test setup is shown in Figure 4.57 and consisted of the harvesting pads connected to the oscilloscope, which in turn is connected to a laptop for data collection. It is noted that, similar to the previous test, the harvester was oriented such that while driving forward, the vehicle would compress the solid transducer harvesting pad first, however this does not seem to affect the harvesting pads performance.

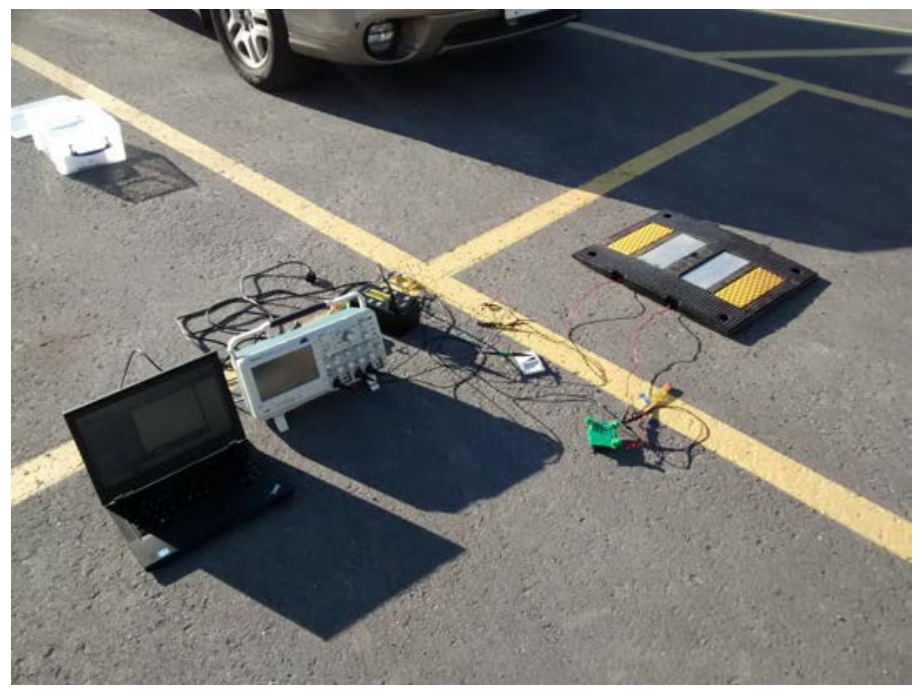

Fig. 4.57: Vehicle speed test setup.

Six trials were performed at varying speeds. The traces were measured separately in 
order to calculate the speeds for each test run. The peak voltages are recorded and plotted against the vehicle speed in Figure 4.58. The peak voltages produced by the harvesting pads with foam padding are 356-290 V for the Cymbal transducers, and $142-108 \mathrm{~V}$ for the preloaded and slotted transducers. These tests imply that the preloaded and slitted transducers are not significantly affected by the speed of the vehicle.

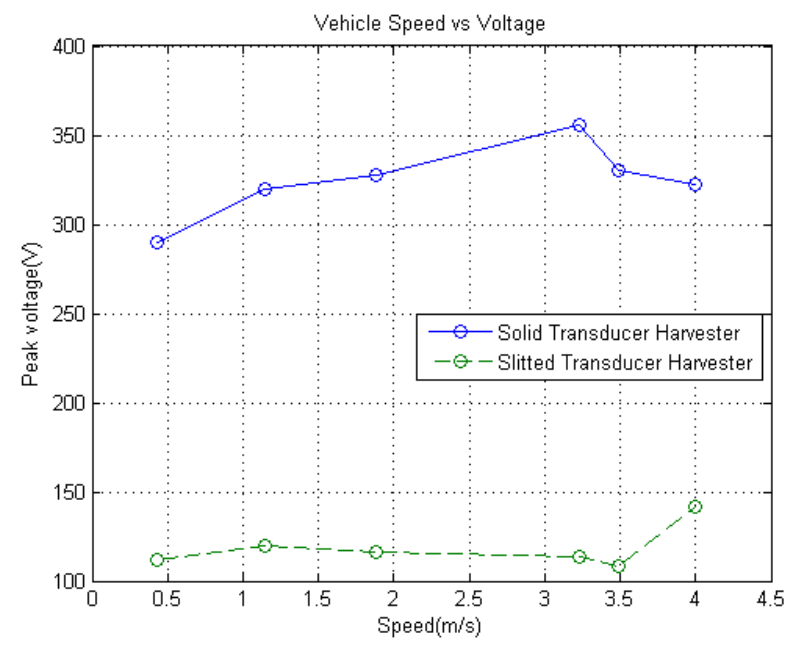

Fig. 4.58: Peak open circuit voltages vs speed.

This set of experimental trials shows a significant decrease in peak voltage output for the preloaded and slitted transducers compared to the previous round of testing as shown in Figure 4.58. This may be due to degradation of the preloaded transducers or incorrectly set preload band stresses. The tests were repeated after removing the pads and their traces are shown in Figure 4.59. It appears that the foam padding had little to no effect on the peak voltage production but may still make the entire harvester more robust as the foam better distributed the transducer loads. 

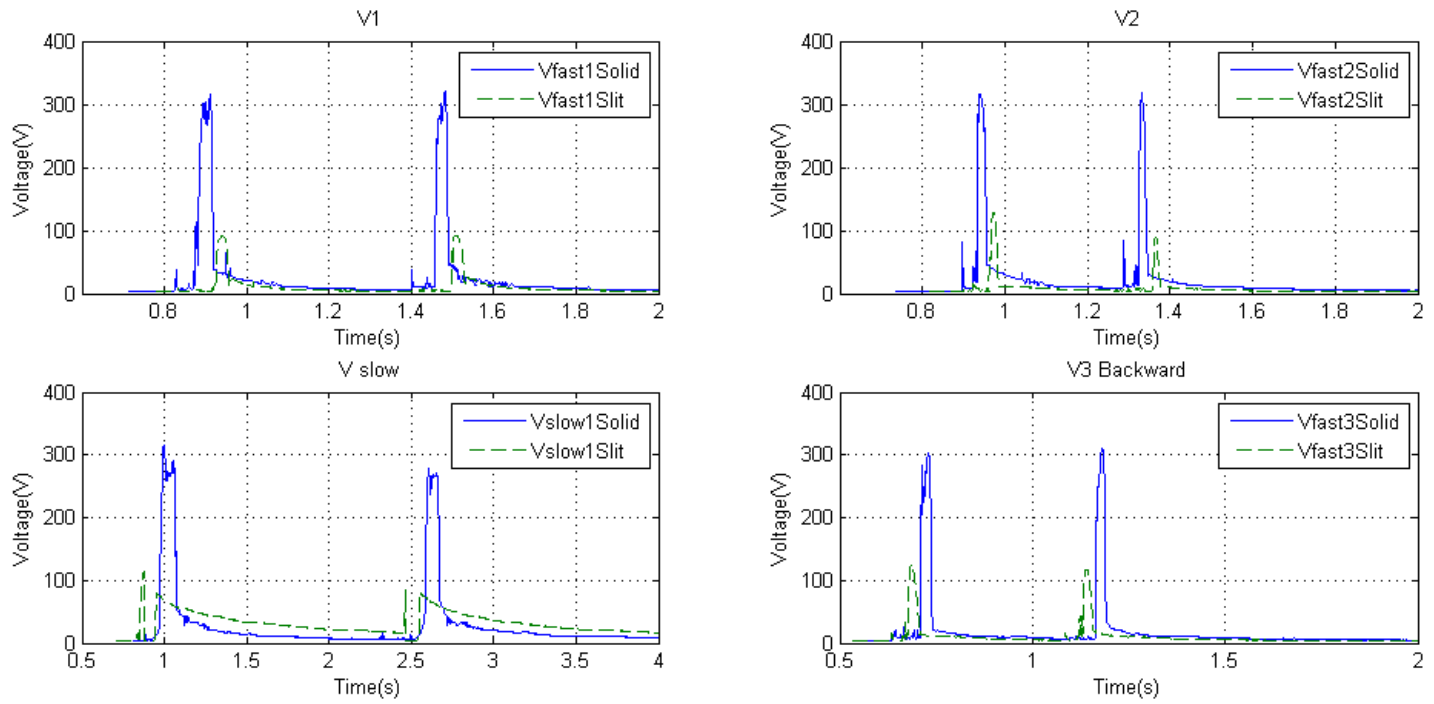

Fig. 4.59: Traces from the experimental transducers with foam padding removed.

Figure 4.60 also indicates that there is no correlation between speed and peak voltage produced. This means that the earlier experimental findings correlating speed to voltage output are an anomaly. The peak voltages produced by the harvesting pads without foam padding are $320-310 \mathrm{~V}$ for the Cymbal transducers, and $128-94 \mathrm{~V}$ for the preloaded and slotted transducers. This could be caused by too much preload tension applied to the slitted cymbal units and so this is explored in the following test. 


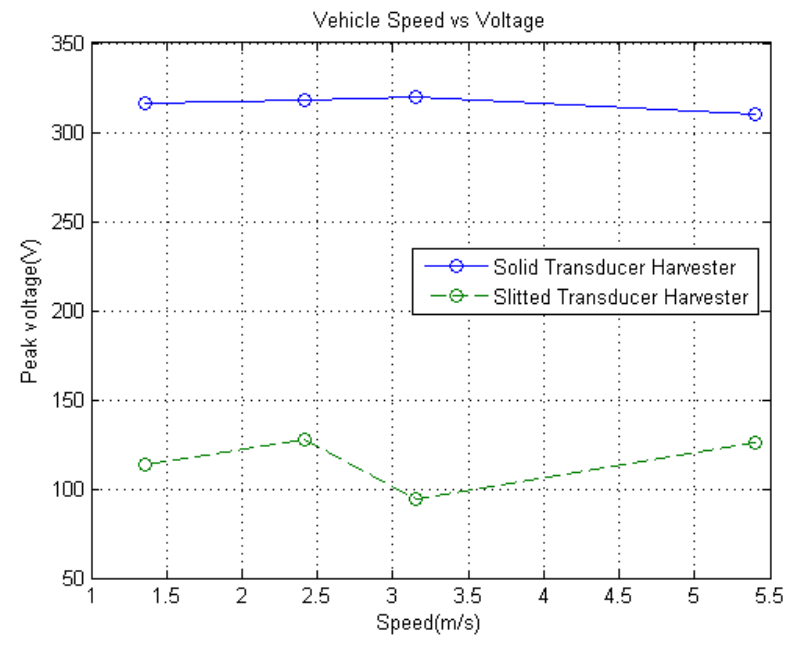

Fig. 4.60: Peak open circuit voltages vs speed with foam padding removed.

Comparison between the foam and no foam results indicate that the foam padding did have a small positive effect on transducer performance, on average improving the output by $16 \mathrm{~V}$. This foam padding is added to the final prototype due to its potential benefits.

\subsubsection{Reducing preload tension}

Since external environments where power harvesting would be implemented experience precipitation, there became a need to make the harvesting pads water resistant. The harvesting pads were encased in large Ziploc ${ }^{\circledR}$ bags with only a small opening for the power wires as shown in Figure 4.61. 


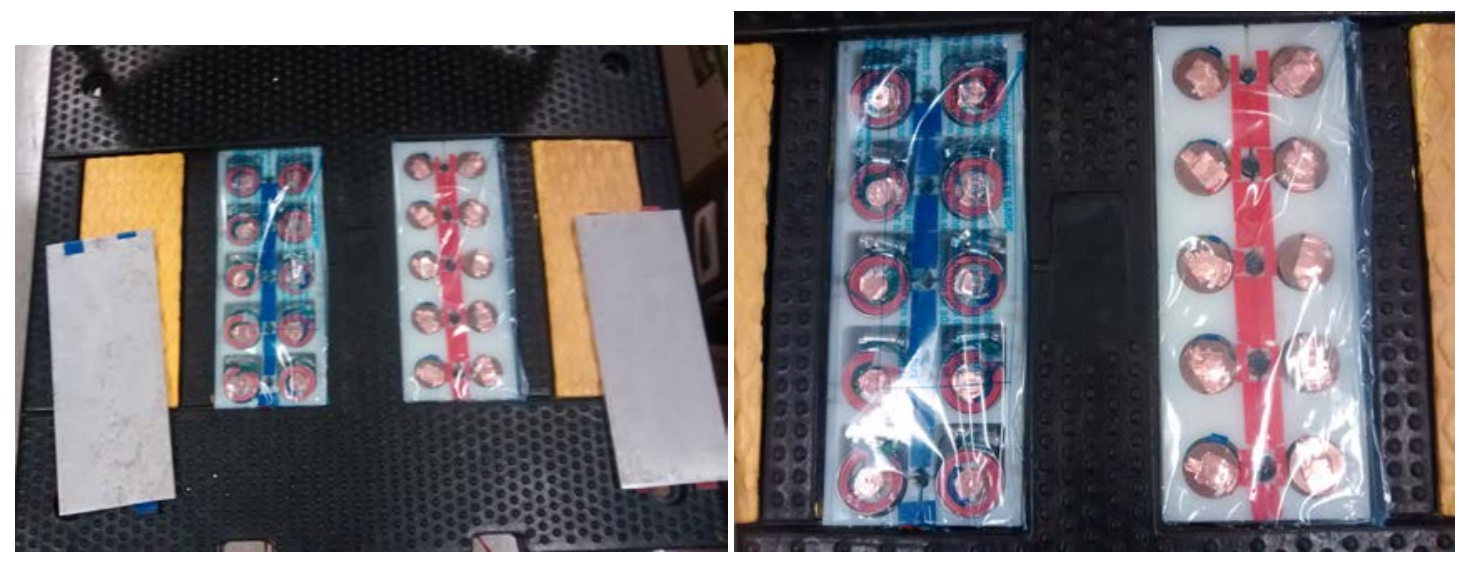

Fig. 4.61: Waterproofed harvesting pads.

The transducers were also disassembled and their electrical connections reinforced with additional conductive epoxy, as some connections could have been degrading. Other transducers had begun shifting from the horizontal stacked configuration similar to the shifting seen in Figure 4.47 and were repaired. The heights of the transducer pairs were checked and found to have been varying by measurable amounts, perhaps causing some transducers to be loaded much more than others. Stainless steel discs, $0.008 "(0.2032 \mathrm{~mm})$ thick, were cut and stacked under the transducers to set them all at approximately the same height $\pm 0.1016 \mathrm{~mm}$, to bring their average height above the harvesting container to $1 \mathrm{~mm}$. Finally, the preload tension was reduced for all transducers to potentially increase their output power. Four experimental trials of differing speed runs were undertaken and oscilloscope traces taken shown in Figure 4.62 and numerical results shown in Table 4.7. The trials were named "V1", "V2", "V3", and, "V4" in order from slowest vehicle movement speed to fastest. 

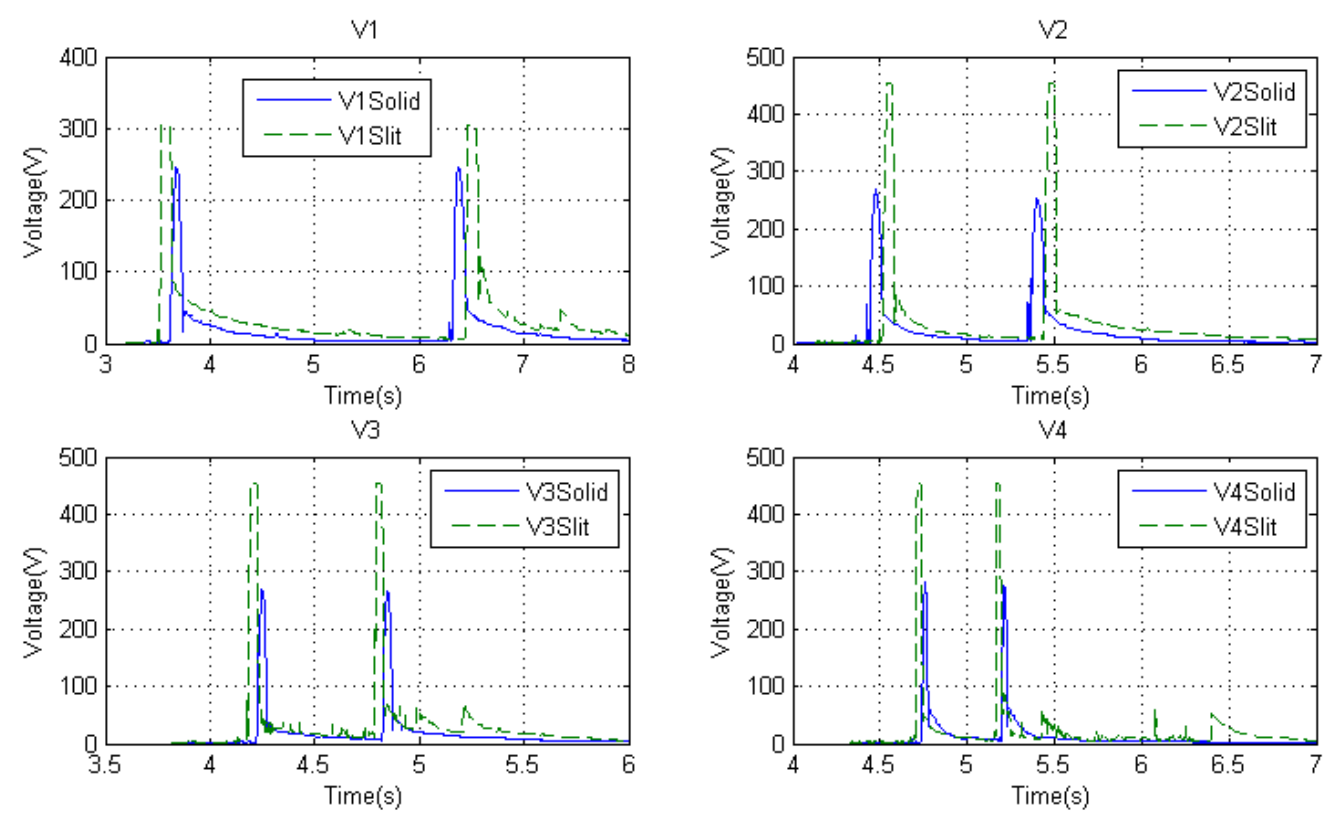

Fig. 4.62: Reduced preload traces.

Table 4.7: Peak voltage attained by transducers using reduced preloading tension.

\begin{tabular}{|c|c|c|c|}
\hline Trial & Average speed & Harvester pad & Peak voltage measured \\
\hline \hline \multirow{2}{*}{ 1 "V1" } & \multirow{2}{*}{$5.63 \frac{\mathrm{Km}}{h}$} & Solid & $247 \mathrm{~V}$ \\
\cline { 3 - 4 } & \multirow{2}{*}{ 2 "V2" } & Slitted and preloaded & $304 \mathrm{~V}$ \\
\hline \multirow{2}{*}{ 3 "V3" } & \multirow{2}{*}{$11.6 \frac{\mathrm{Km}}{\mathrm{h}}$} & Solid & $268 \mathrm{~V}$ \\
\cline { 3 - 4 } & & Slitted and preloaded & $454 \mathrm{~V}$ \\
\cline { 3 - 4 } 4 "V4" & \multirow{2}{*}{$17.3 \frac{\mathrm{Km}}{h}$} & Slitted and preloaded & $270 \mathrm{~V}$ \\
\cline { 3 - 4 } & & Slitted and preloaded & $454 \mathrm{~V}$ \\
\hline
\end{tabular}

The results show the solid and preloaded transducers far out performing their solid endcapped counterparts, with $454-304 \mathrm{~V}$ compared to the $282-247 \mathrm{~V}$ peaks from the cymbal transducers. Small voltage spikes, separate from the main spikes in traces "V1", "V3", and "V4" indicate that the transducers are experiencing damage. After 20 to 30 more vehicle compressions were performed, for the testing of a harvesting circuit, a final open circuit test was completed shown in Figure 4.63. There appears to be slightly reduced peak voltage from both harvesting pads in this last degradation 
test which could also be caused by the vehicle not being centered properly on the harvesting pads rather than confirmation of transducer degradation for both harvesters simultaneously.

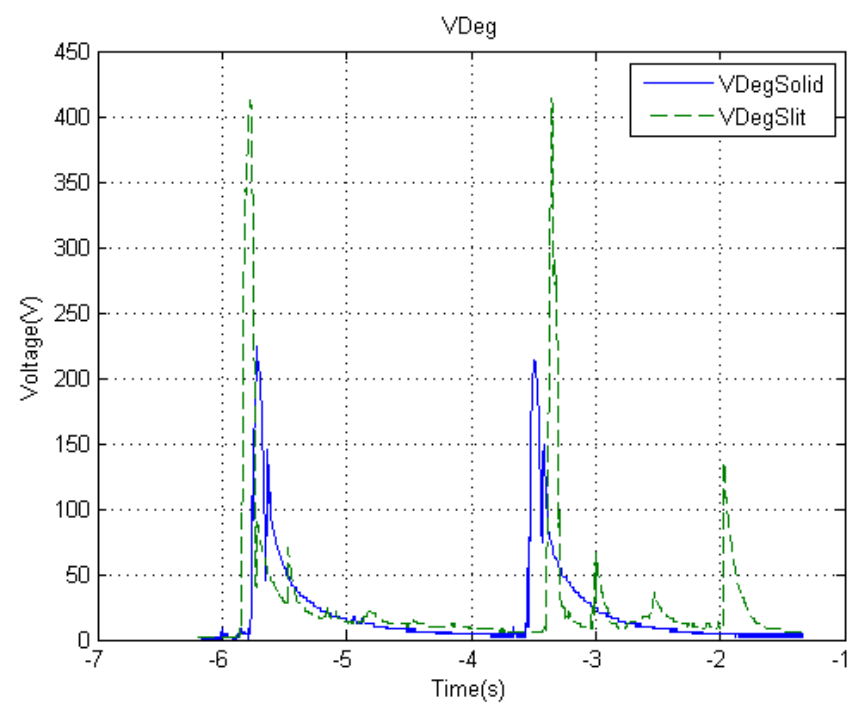

Fig. 4.63: Short term degradation test.

The speeds and peak voltages shown in Table 4.7 are plotted against each other and are shown in Figure 4.64. From Figure 4.64, it can be concluded that vehicle velocity, at the speeds tested, is not a significant factor on peak voltage production.

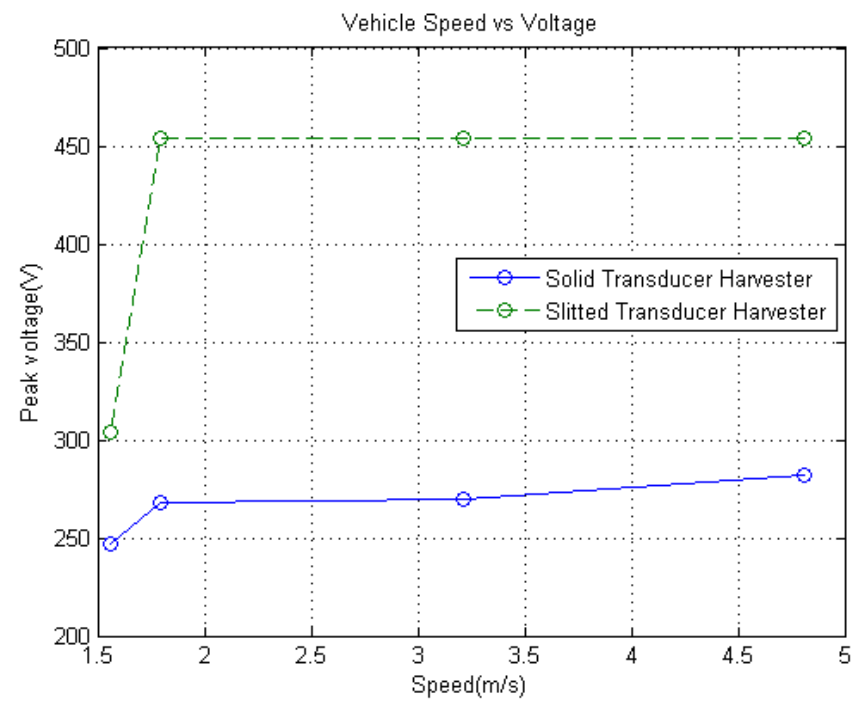

Fig. 4.64: Peak voltages plotted against speed for the reduced preload tests. 
It now appears that the power production is much higher for the slitted preloaded transducers as their peak voltages are consistently higher at all speeds. Three of the four tests returned a peak voltage value of $454 \mathrm{~V}$ which seems to be the peak voltage that the oscilloscope can measure. The oscilloscope itself is rated to $400 \mathrm{~V}$. Given the oscilloscope measurement limitations and the identical peak voltages shown in Figure 4.64, it seems likely that the measured voltages have been truncated to $454 \mathrm{~V}$. It should also be noted that the bridge rectifiers connecting the transducer pairs to the main output wires are rated for a maximum reverse voltage of $400 \mathrm{~V}$ necessitating an upgrade for the bridge rectifiers.

\subsubsection{Transducer damage}

It was noticed after the reduced preload tension tests that some of the traces collected such as Figures 4.62 and 4.63, showed odd voltage spikes after the main vehicle compression and release. This behavior was seen before in earlier tests as seen in Figure 4.55 but was ignored as anomalous noise. Qualitatively examining transducers and their traces, it was found that some pairs produced jagged traces which corresponded to apparent cracks in the PZT material. Some examples of damaged transducer traces are shown in Figure 4.65 and an example of a damaged transducer is shown in Figure 4.66. The traces were produced by finding transducers with apparent cracks and manually applying a force to the unit to examine the trace. 

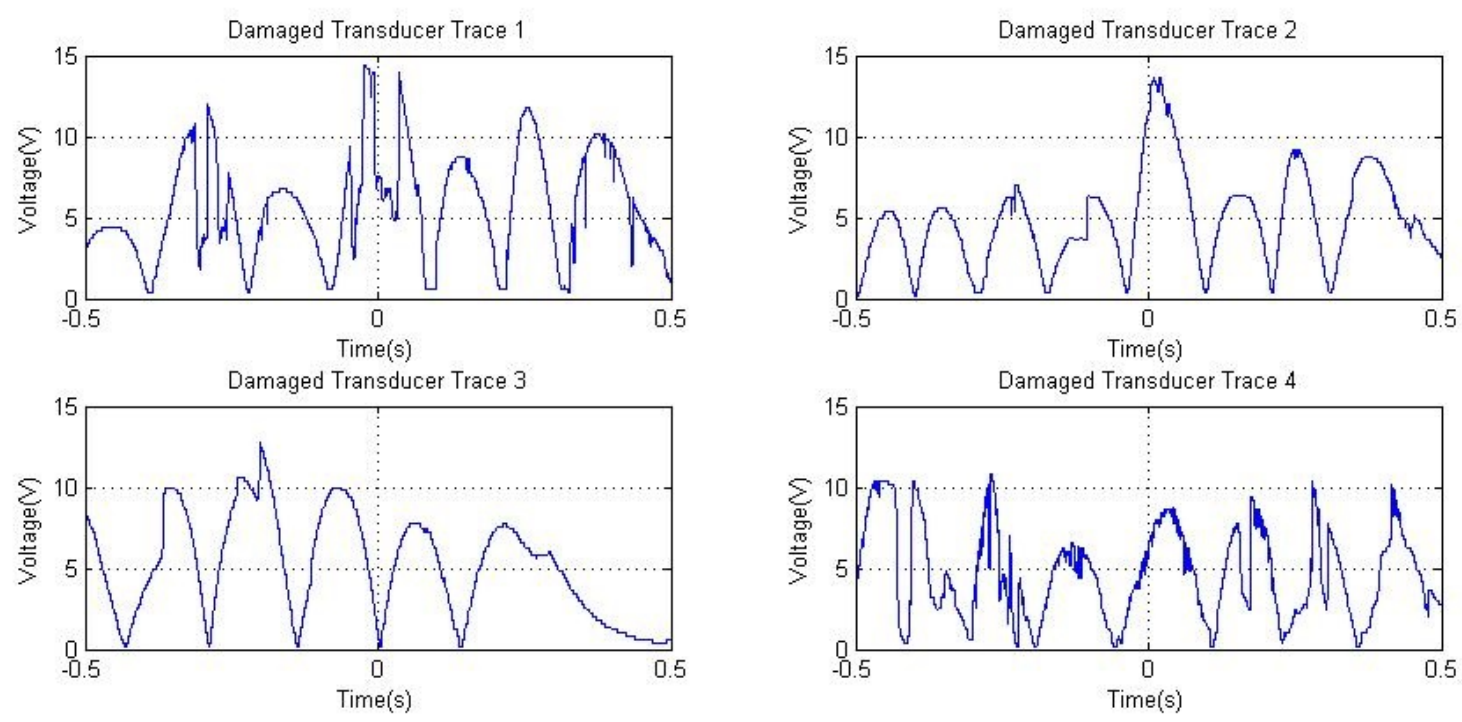

Fig. 4.65: Damaged transducer traces.

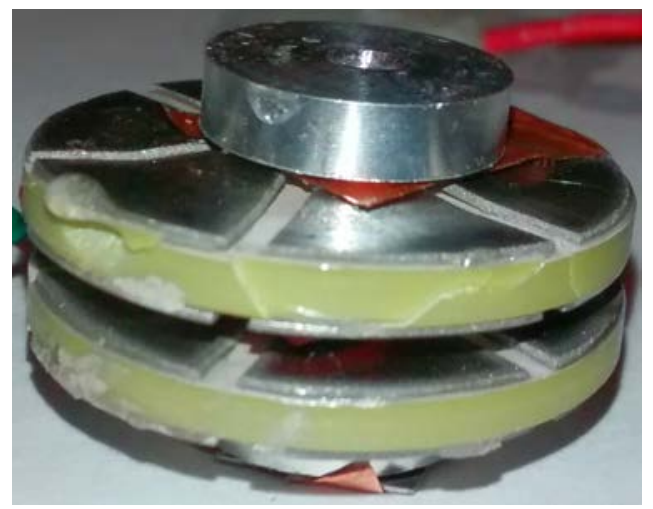

Fig. 4.66: Damaged transducer in a pair.

The damaged transducers were taken apart for examination and the piezo disc from the damaged transducer shown in Figure 4.66 is shown in Figure 4.67. In order to continue testing, three transducers were taken apart and replaced with new ones as there was only enough leftover piezo material to allow for three more replacement units to be manufactured. 


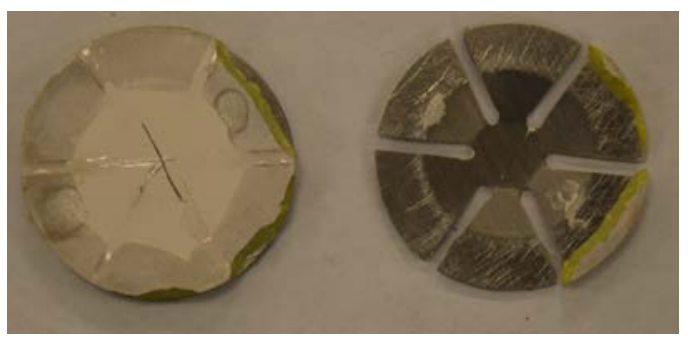

Fig. 4.67: Damaged piezo disc from Figure 4.66.

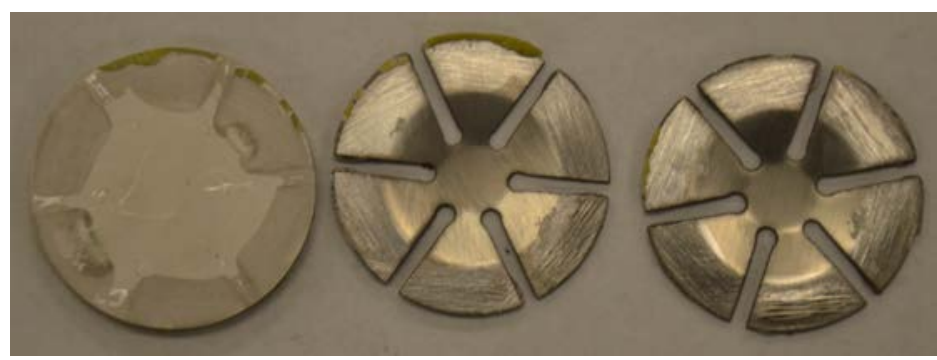

Fig. 4.68: The second damaged transducer disassembled.

The three transducers to be replaced were ones that showed the highest amount of cracking damage around the outside edge. The second of the three transducers taken apart had damage similar or exactly to that of Figure 4.67 shown in Figure 4.68, while the third piezo transducer suffered much deeper cracking which appeared to associate with the slitted end caps discrete contact areas and is shown in Figure 4.69. These damage patterns, which seem to follow and be caused by the slitted end caps, appear very different from other damage tests performed on the MTS ${ }^{\circledR}$ machine using solid transducers were there was a tendency of piezo units to shatter or crack through the center.

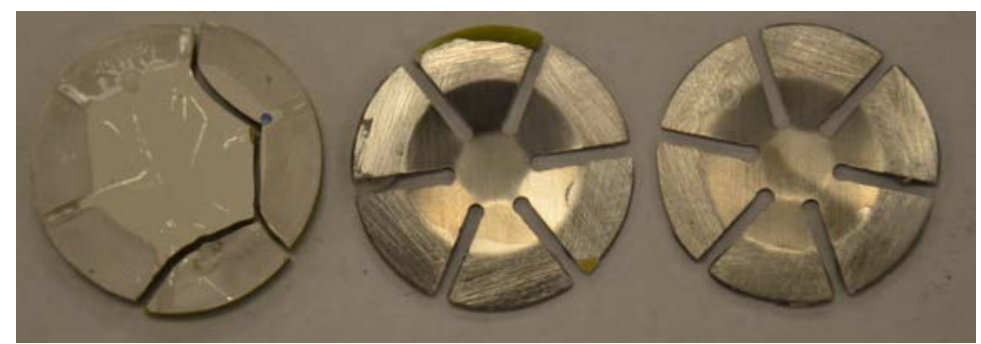

Fig. 4.69: The most heavily damaged piezo unit examined. 
The damaged transducers are still be capable of generating significant voltages due to the relaxation of their preloading when a vehicle load is applied to the transducer pair however their potential is lowered as they cannot generate power from tension. While unloaded, the damaged transducers will be under compressive loading from their preloading bands. The relaxation of this compressive loading from the external transducer loading would still cause the PZT fragments to generate a proportional electric field, thereby producing electricity. This mechanism could only work up to the point when the radial stress changes sign from compressive to tensile, in which case the PZT fragments would separate and no more power would be generated.

\subsection{Full scale roadway harvester}

In order to demonstrate the roadway power harvesting system, a full scale roadway power harvester was designed and constructed.

\subsubsection{Full scale harvester design and fabrication}

The updated system uses two speed bumps, with four harvesting pads in each for a total of eight harvesting pads. The nylon container design was modified to allow built in spacers at the bottom of the individual cells to make contact with each transducer. Holes for the bridge rectifiers where also moved in a more convenient location on the harvesting pad and enlarged, which are shown in Figure 4.70. The harvesting pad was

machined on a Computer Numeric Control (CNC) machine and as such was designed using the parameters (shown in Figure 4.70) which are defined in inches. 


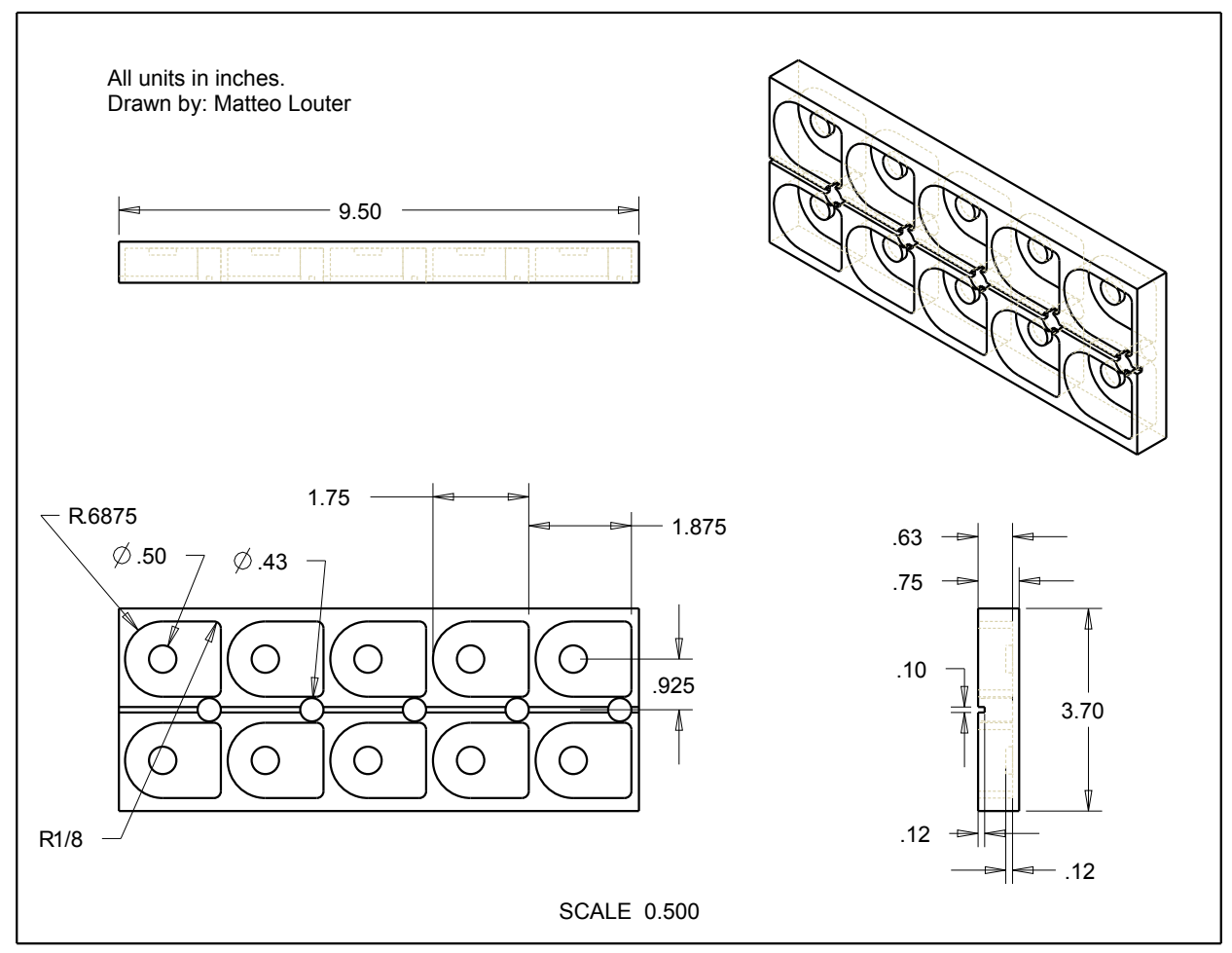

Fig. 4.70: Improved harvesting pad housing design.

The design called for eight harvesting pads with ten transducer pairs each totaling 80 transducer pairs or 160 individual transducers which were hand assembled using methods previously described and are shown in partial assembly in Figure 4.71.

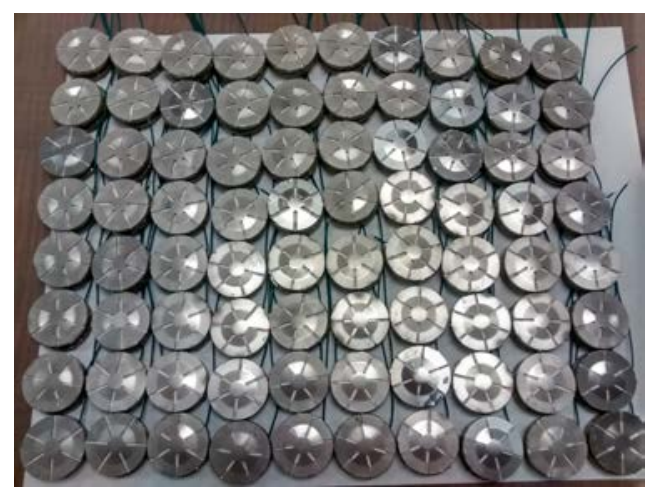

(a) All 80 transducers paired off and under assembly.

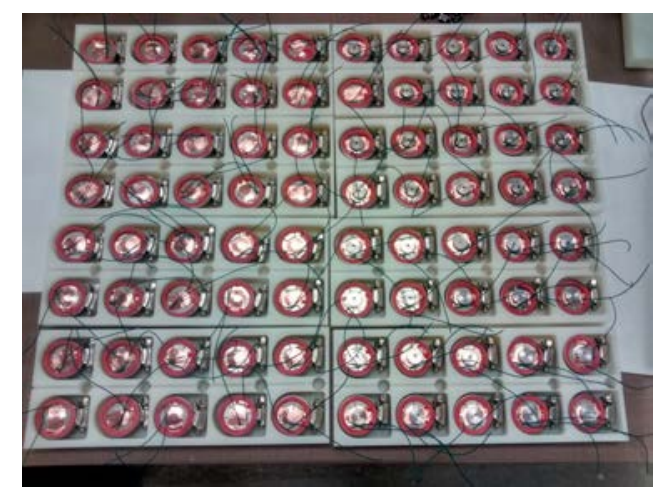

(b) Transducer pairs assembled, preloaded, and placed in the nylon housings.

Fig. 4.71: Transducers at various stages of assembly.

The cover panels for the harvesting pads were covered in black and yellow anti-slip 
grip tape which although may wear quickly in actual use, gives a good aesthetic and traction for technology demonstrations involving the power harvesting units. Each harvesting pads cover plate is attached to the pad with a strip of electrical tape which also assists in keeping water out of the harvesting pad. Finally rubber pads made from two layers of the same bands that the preloading buffer bands are made out of were added to the top of each transducer in order to compensate for small variations in transducer height and to increase the height above the $0.4 \mathrm{~mm}$ displacement estimate given by Figure 3.20. An image of a completed harvesting pad next to its cover plate and fully assembled with electrical tape are shown in Figure 4.72.

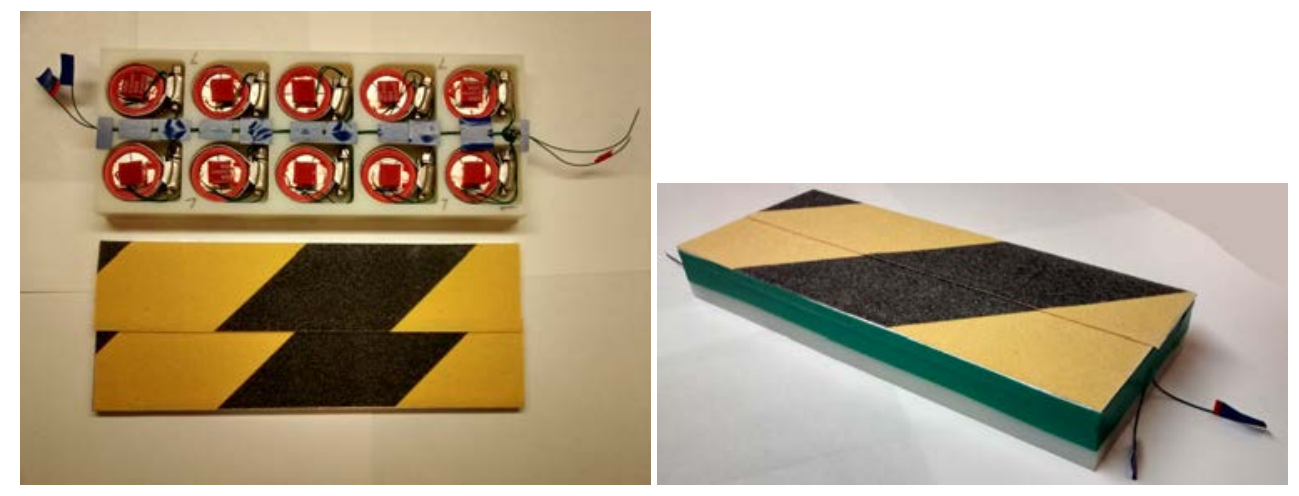

Fig. 4.72: A single harvesting pad wired and sealed with electrical tape.

The two speed bumps used are Barco standard speed hump sections. These speed humps are very similar to the one used previously, however it is elongated which allows for four harvesting pads to be embedded in the hump. Due to the humps increased thickness, wooden blocks are used to support the harvesting pads and keep them aligned with the top of the speed hump. The wooden blocks are held in the speed hump with truss connector plates and are shown in Figure 4.73. 


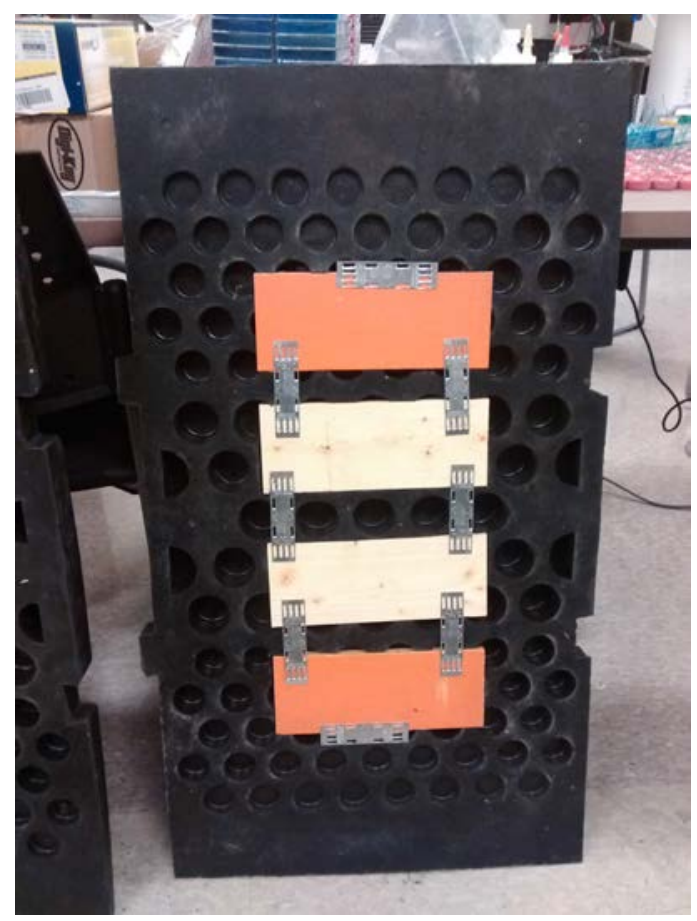

Fig. 4.73: Speed bump with truss connector plates used to attach the wooden spacers.

Figure 4.74 shows one of the two speed humps before and after installation of the harvesting pads. Holes were drilled into the sides of the speed humps to route the cables which carry the harvested power to a power conditioning circuit.
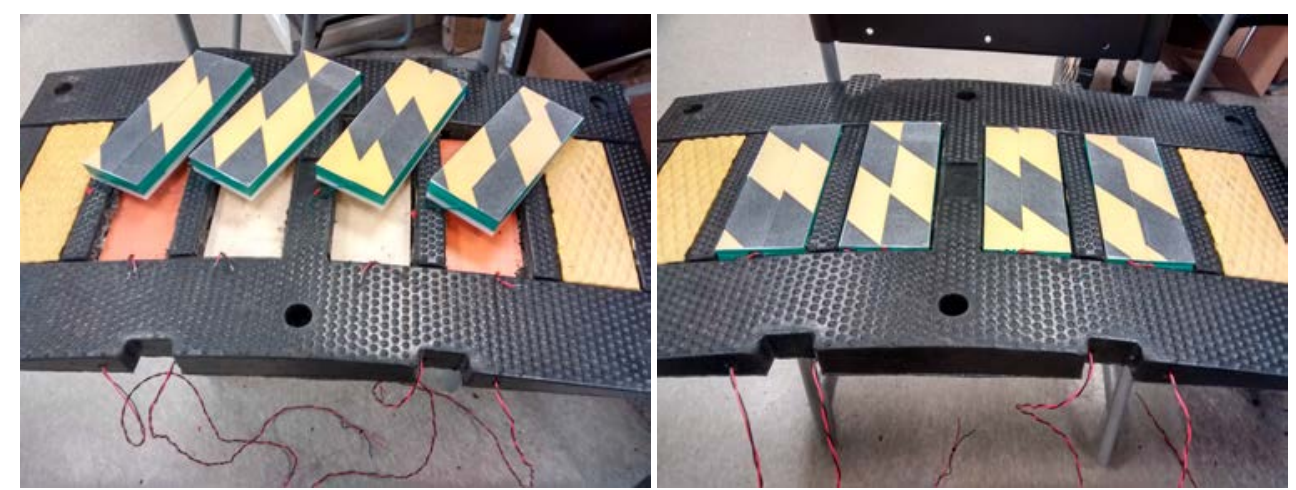

Fig. 4.74: Harvesting pads on top of and placed in the speed bump. 


\subsubsection{Full scale harvester test}

The test setup was similar to those of previous tests and used one of the two harvesting pads as they have been constructed identically. An extension cable was used to power the Tektronix ${ }^{\circledR}$ DPO 2024B oscilloscope and a laptop used to record the trace data. Figure 4.75 shows the testing setup with the test vehicle, the same one used on all other tests, barely visible in the top right corner of the image. For referencing purposes, the harvesting pads were given numerical designations 1 - 4 from left to right as shown in in both Figure 4.74 and 4.75 .

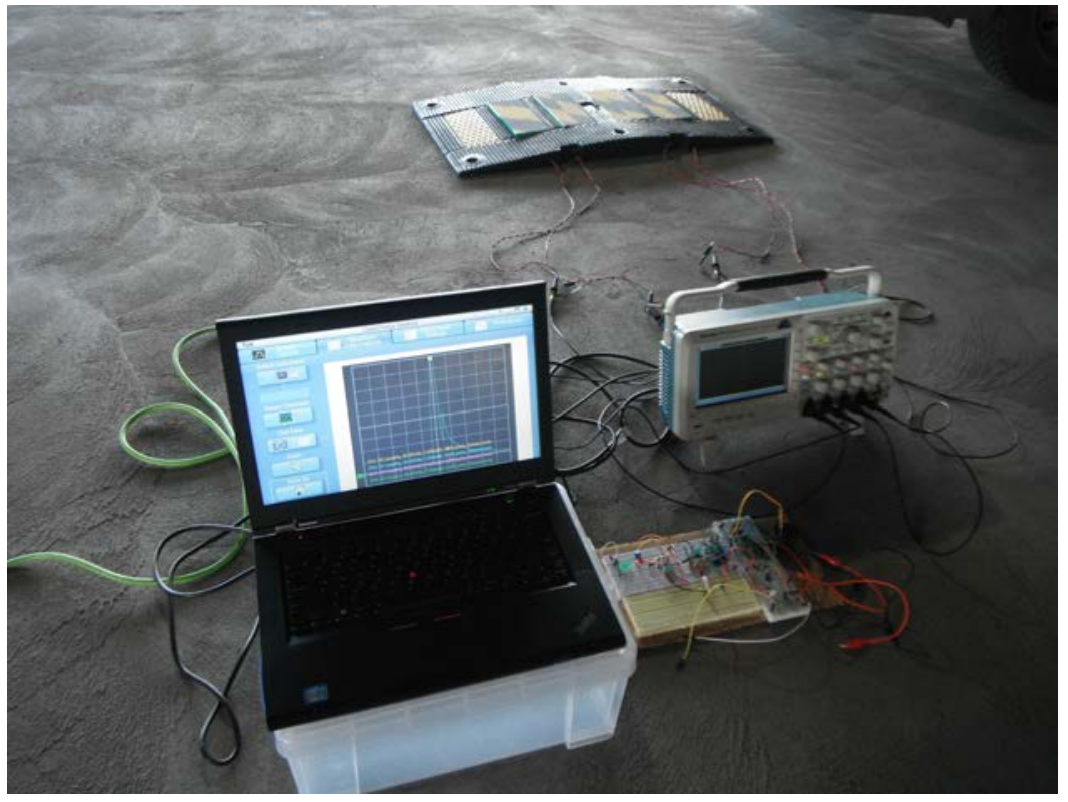

Fig. 4.75: Full scale harvester test setup.

An initial test was performed with all harvesting pads, except harvesting pad 2, removed. The resulting trace taken is shown in Figure 4.76. The peak voltage exceeds the oscilloscopes ability to measure it and far exceeds the voltage probes peak rated voltage of $300 \mathrm{~V}$. 


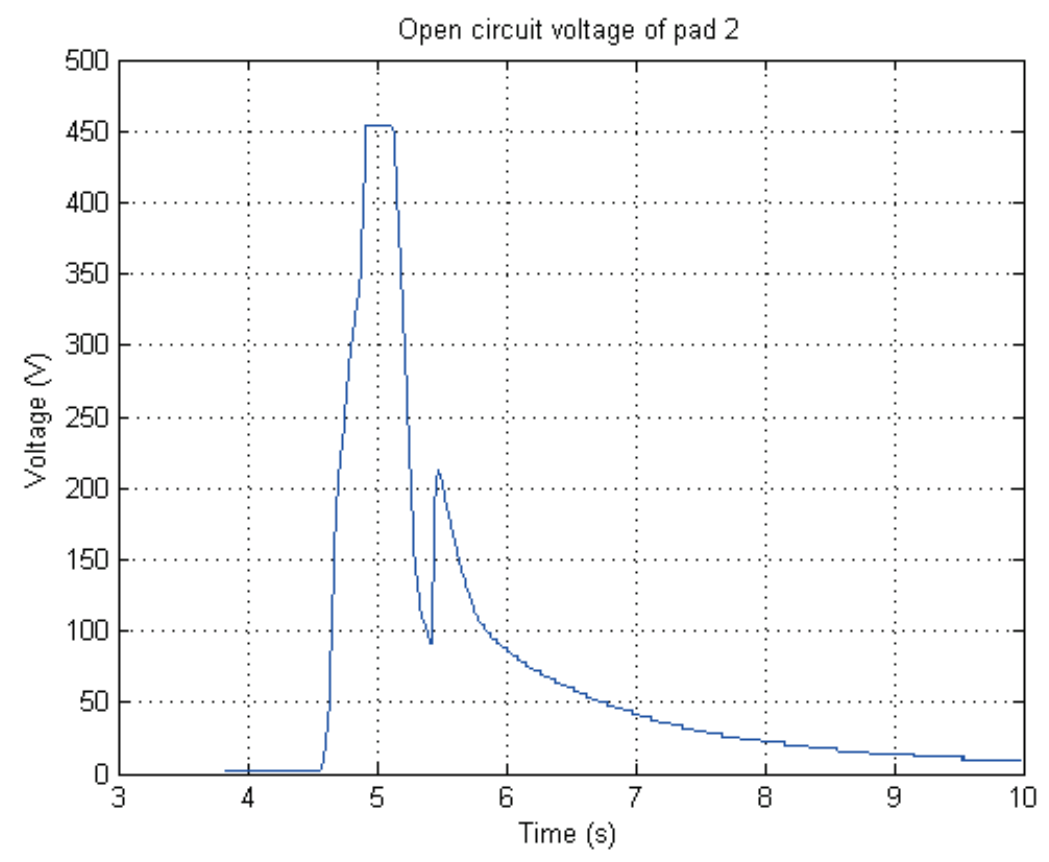

Fig. 4.76: Initial compression with only harvesting pad 2.

Because of this, four voltage dividers where made in order to half the voltage that the oscilloscope was receiving from all four harvesting pads. Since the voltage probe has a resistance of $10 \mathrm{M} \Omega$, resistors were chosen such that the resistance of the voltage divider was also $10 \mathrm{M} \Omega$ so as not to affect the test results. The schematic for a single voltage divider attached to a single harvesting pad is shown in Figure 4.77.

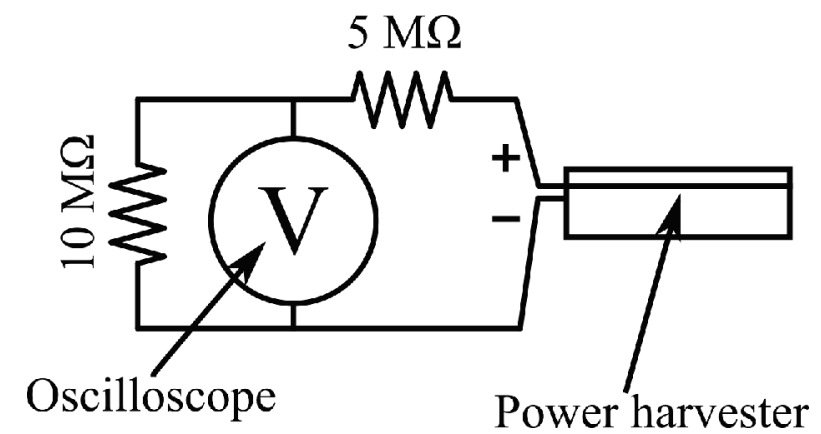

Fig. 4.77: Schematic for a voltage divider used to allow measurement of higher voltages.

Figure 4.78 shows the calculated traces from traversing the harvesting pad with the 
front wheel of the same vehicle used in all tests. The recorded traces were simply doubled to extrapolate the actual voltages produced by the harvesting pads.

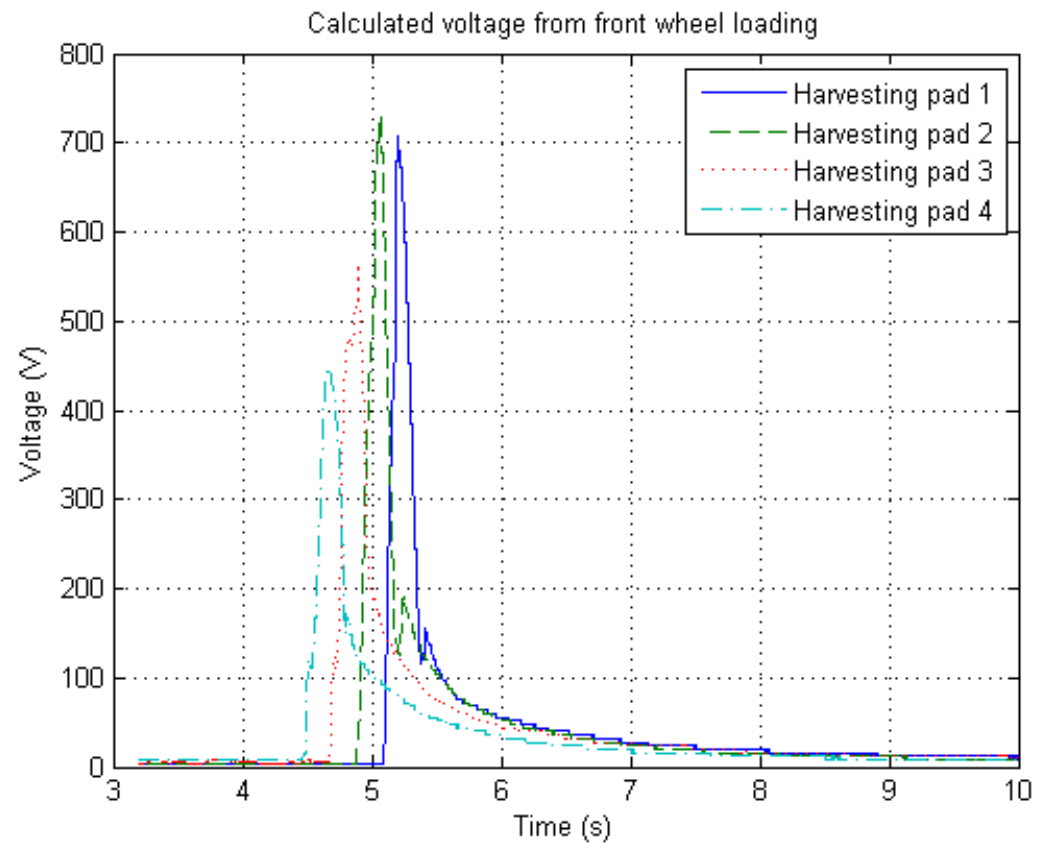

Fig. 4.78: Calculated full scale traces from initial front wheel loading.

Seven, 20 pound weights where added incrementally to the vehicle up to 140 pounds, between the rear wheels to examine the effect that vehicle weight would have on the harvesting pads output. Figure 4.79 shows the placement of the weights in the test vehicle. 


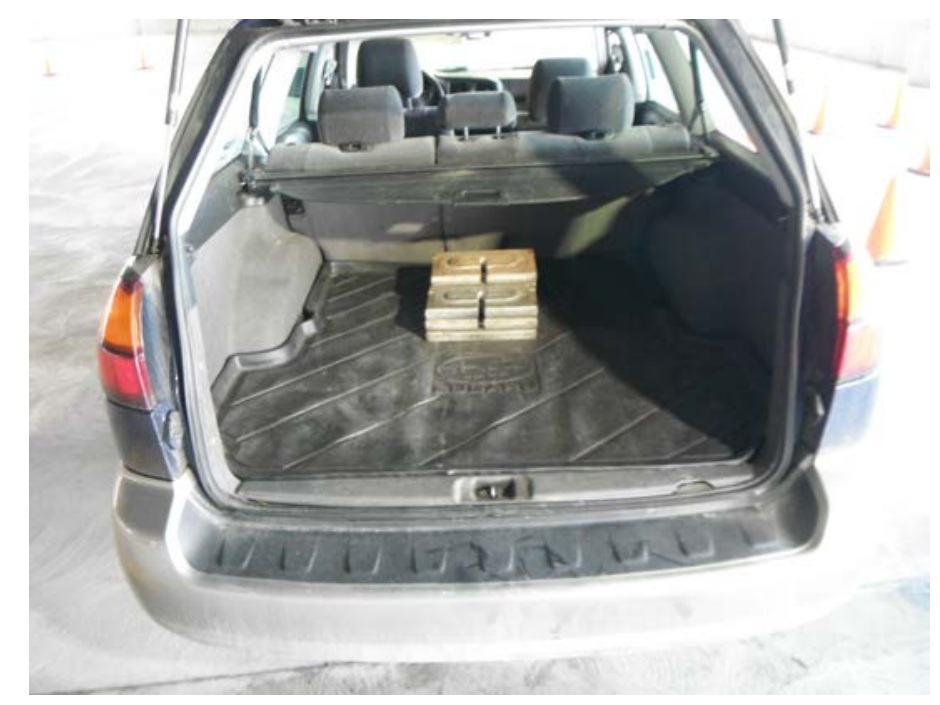

Fig. 4.79: All seven, $20 \mathrm{lb}$ weights placed in the rear of the test vehicle.

The results from the rear wheel loading tests reveal several points. Figure 4.80a and $4.80 \mathrm{~b}$ show the extrapolated voltage traces for rear wheel loading at 0 and 20 additional pounds. It can be seen that the peak voltages change in a way that indicates that the driving direction plays a significant role in the peak voltage production. Furthermore by comparing a loading scenario using the heavier front wheel (shown in Figure 4.78) to the rear wheel with no additional load (shown in Figure 4.80a) it can be seen that very similar peak voltages are produced, indicating that the transducers are compressed fully and additional loading force is being absorbed by the transducers protective housing. The vehicle loading in the two scenarios mentioned previously are applied with the vehicle traveling in the same direction further indicating that weight has little effect and that loading direction has a much more significant effect. 


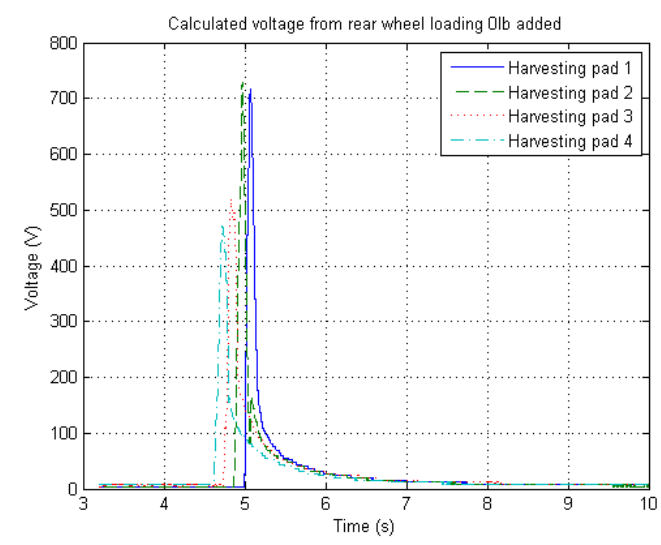

(a) Calculated voltage trace with 0 pound wheel loading.

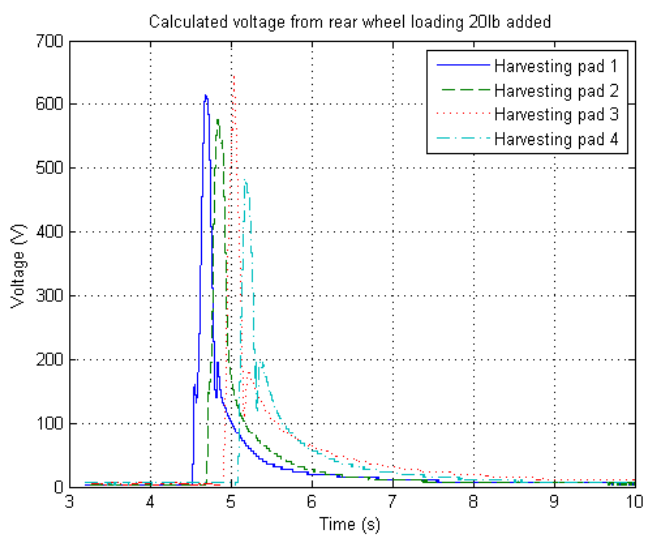

(b) Calculated voltage trace with 20 pound wheel loading.

Fig. 4.80: Calculated voltage traces for rear wheel loading scenarios.

Figure 4.81 plots the calculated peak voltages over the ranges of additional loading's tested and shows no significant or consistent change caused by increased weight. Comparing the two sub figures together however (Figure 4.81a and 4.81b) shows a large difference caused by the direction the vehicle traversed the harvesting pads. This may be caused by the harvesting pads protruding from the speed bump at their edges, a phenomenon caused by the horizontal mounting of the pads, and the speed bumps curved driving surface.

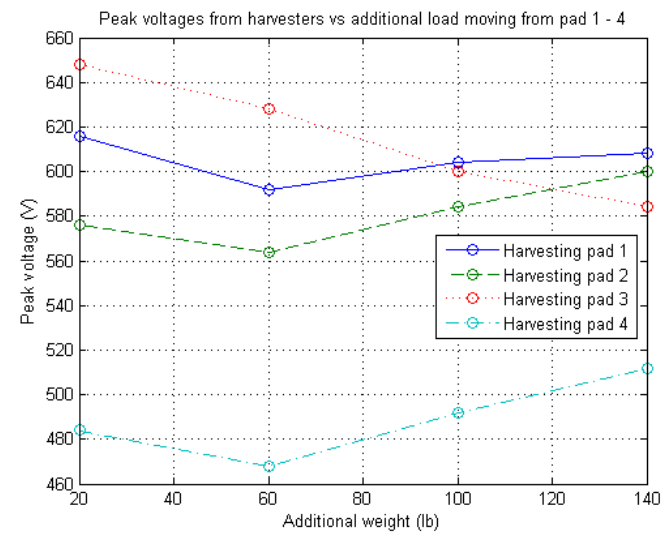

(a) Calculated peak voltages for vehicle motion from pad 1 to pad 4.

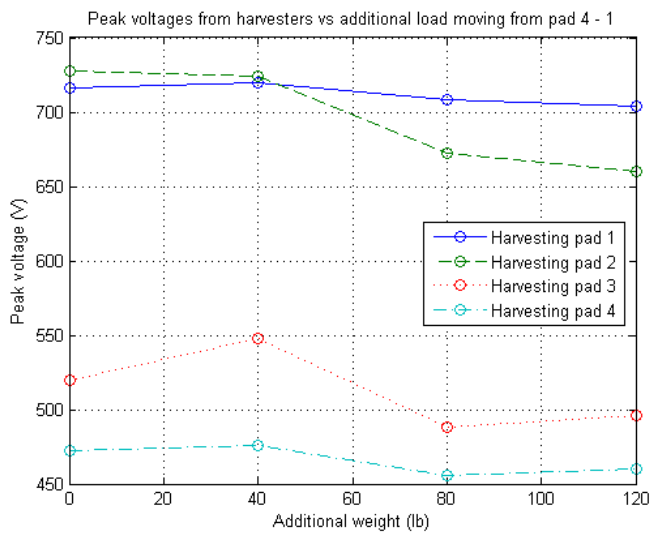

(b) Calculated peak voltages for vehicle motion from pad 4 to pad 1.

Fig. 4.81: Calculated peak voltages from all harvesting pads plotted against the added mass for rear wheel loading scenarios. 
Finally, measurements where taken at various vehicle speeds to examine the effect speed may have on the new full scale harvester. Figure 4.82 plots the average peak pad voltage against the speed measured by comparing voltage rise times and knowing the pad separation distance. From this test, it does not appear that speed significantly affects the power harvesters output.

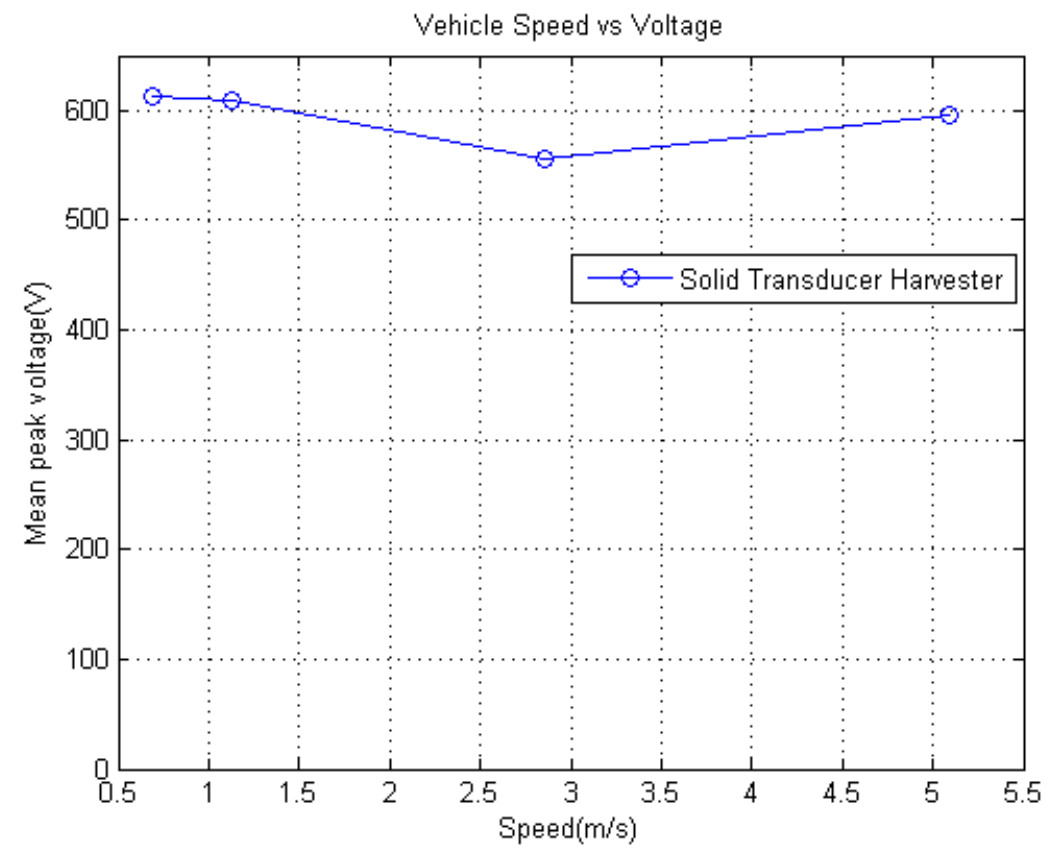

Fig. 4.82: Vehicle speed vs average peak harvesting pad voltage.

Using Equation 2.2 and the average peak voltages displayed in Figure 4.81, the total possible energy per tire compression can be calculated as:

$$
\frac{1}{2} C_{\text {total }} V_{\text {avg }}^{2}=\frac{1}{2} \cdot 6.33 \times 10^{-7} \mathrm{H} \cdot 584 \mathrm{~V}=0.108 \mathrm{~J}
$$

Assuming a daily traffic value of 150,000 , each vehicle having a single front and rear tire, the single harvesting pad is calculated to, on average, continuously output:

$$
300,000 \frac{1}{d a y} \cdot 0.108 \mathrm{~J}\left(\frac{1}{24} \frac{d a y}{h}\right)\left(\frac{1}{60} \frac{h}{\min }\right)\left(\frac{1}{60} \frac{\min }{s}\right)=0.375 \mathrm{~W}
$$


And with both pads operating together, an average ideal output would be $0.751 \mathrm{~W}$. This value is much higher than the estimation made at the end of Chapter 2 due to the much higher than expected peak voltages, however inevitable inefficiencies in any harvesting circuitry would reduce the usable power output.

This experiment demonstrates the improved power production capabilities of radially slitted and preloaded piezoelectric cymbal transducers applied to roadway power harvesting. Peak voltages recorded for the solid, non slitted and non preloaded transducers were $344 \mathrm{~V}$ less than the peak voltage from the novel transducer design at $732 \mathrm{~V}$. This represents a $113 \%$ increase in peak voltage production indicating that preloading and radially slitted designs may be the next way forward in roadway power harvesting. 


\section{Conclusion}

\subsection{Summary}

Cymbal transducers continue to be a viable option for roadway power harvesting due to their simplicity, ease of manufacture and lack of actuating joints. This thesis has demonstrated design and construction of a radially slitted and preloaded transducer, designed for roadway power harvesting applications. The transducers are bundled into a power harvesting speed bump system capable of generating and storing electricity by way of vehicles compressing the bump. The system is then capable of delivering the stored energy via a power conditioning circuit to an arbitrary application at a specified voltage. By applying a radial compressive preload we are able to improve the damage resistance of standard Cymbal piezoelectric transducers. This compensates for the increased radial tensile loading of higher compressive loading situations. Combined with a radially slitted Cymbal end cap design, the new transducer can output up to $732 \mathrm{~V}$ which is $113 \%$ more power than a conventional cymbal design. These new transducers, though larger and more complex to manufacture, are specifically designed for high load and higher power applications. These improvements also reduce the number of transducers needed for a specific application as the transducers that do exist apply a larger range of strain to their piezoelectric materials and can generate more power.

In addition it was found that by examining voltage rise times between sequential harvesting pads, the vehicles speed could be measured. Further exploration of this ability could lead to automated vehicle speed monitoring which wouldn't require additional roadway sensors and be an added feature to any roadway power harvesting system. Addition of a speed monitoring feature would require additional circuitry though such circuitry could be integrated into the existing PCB so that the compact 
nature of the integrated circuit board could be maintained.

\subsection{Recommendations for future work}

Improvements to the mechanical design of the roadway power harvesting transducers are as follows. Improvements could be made to manufacturing quality, by improving the type and thickness of the buffer rubber applied between the transducer and preload band. The preload band itself can be improved by designing a mechanism to allow the band to expand and contract with the transducer while applying a specific and continuous preload force. This improvement would also assist in estimating and setting a desired preload tension without need for strain gauges.

Improvements to the hardware prototypes include improvements to the securing method when installing pads in the rubber speed bump and improvements in terms of more weather resistant and waterproof harvesting container sealing. In addition, custom speed bumps could be designed to maximize the number of harvesting pads that they could accept. Methods for implanting the roadway harvesting pads into the road surface also still needs to be addressed including installation and wiring methods. Finally the design of the housing can be further improved by exploring more efficient transducer pattern layouts and tailoring layout shapes to specific applications and consumer needs.

Final improvements to the parametric design can be made by discarding assumptions made about the independence of parameters on each other and their affect on performance. Black box global optimization such as Kriging can be used in order to fine tune the basic transducer parameters for more specialized applications using FEA models.

Manufacturing processes can also be improved by exploring cheaper methods of cut- 
ting the end cap shapes such as machining or combining punching and cutting which may drastically lower cost especially if producing large batches of transducers. Finally the assembly of the transducers can be improved by using a more uniform and consistent epoxy application method such as spin coating as used in [44], and finding a more consistent way of applying the silver conductive epoxy. 


\section{References}

[1] D. Hill, A. Agarwal, and N. Tong, "Assessment of Piezoelectric Materials For Roadway Energy: Cost of Energy and Demonstration Roadmap," DNV KEMA Energy \& Sustainability, Tech. Rep., 2014.

[2] S. R. Anton and H. a. Sodano, "A review of power harvesting using piezoelectric materials (2003-2006)," Smart Materials and Structures, vol. 16, no. 3, pp. R1-R21, Jun. 2007. [Online]. Available: http://stacks.iop.org/0964-1726/16/i= $3 / \mathrm{a}=\mathrm{R} 01$ ?key $=$ crossref.2c5609f4e60f2ce4b89d0db98aedfe93

[3] C.-C. Ting, D.-Y. Tsai, and C.-C. Hsiao, "Developing a mechanical roadway system for waste energy capture of vehicles and electric generation," Applied Energy, vol. 92, pp. 1-8, Apr. 2012. [Online]. Available: http: //linkinghub.elsevier.com/retrieve/pii/S0306261911006556

[4] H. Zhao, J. Yu, and J. Ling, "Finite element analysis of Cymbal piezoelectric transducers for harvesting energy from asphalt pavement," Journal of the Ceramic Society of Japan, vol. 118, no. 1382, pp. 909-915, 2010. [Online]. Available: http://joi.jlc.jst.go.jp/JST.JSTAGE/jcersj2/118.909?from=CrossRef

[5] P. Kemball-Cook, L. and Tucker, "Energy harvesting," 2013. [Online]. Available: https://www.google.com/patents/US20130068047

[6] H. Abramovich, C. Milgrom, E. Harash, L. E. Azulay, and U. Amit, "Multi-Lyer Piezoelectric Generator," 2012.

[7] H. Abramovich, E. Harash, C. Milgrom, and U. Amit, "Energy Harvesting From Airport Runway," 2009.

[8] H. Abramovich, E. Harash, C. Milgrom, U. Amit, and L. E. Azulay, "Power Harvesting From Railway Aapparatus, System And Method," 2010.

[9] M. S. Vijaya, Piezoelectric Materials and Devices Applications in Engineering and Medical Sciences. Taylor \& Francis, 2012.

[10] J. F. Tressler, W. C. K. Uchino, and R. E. Newnham, "Finite element analysis of the cymbal-type flextensional transducer," IEEE Transactions on Ultrasonics, Ferroelectrics, and Frequency Control, vol. 45, no. 5, pp. 1363-1369, 1998.

[11] V. Piefort, "Finite Element Modelling of Piezoelectric Active Structures," Ph.D. dissertation, Université Libre de Bruxelles, 2001.

[12] R. J. Meyer, W. J. Hughes, T. C. Montgomery, D. C. Markley, and R. E. Newnham, "Design of and Fabrication Improvements to the Cymbal Transducer Aided by Finite Element Analysis," Journal of Electroceramics, vol. 8, pp. 163 - 174, 2002 . 
[13] S. Lin, "An improved cymbal transducer with combined piezoelectric ceramic ring and metal ring," Sensors and Actuators, A: Physical, vol. 163, no. 1, pp. 266-276, 2010. [Online]. Available: http://dx.doi.org/10.1016/j.sna.2010.06.022

[14] Z. Jindong, W. J. Hughes, A.C. Hladky-Hennion, and R. E. Newnham, "Concave cymbal transducers," in Innovations in Materials Conference, Washington, D.C., 1999, pp. 252-255.

[15] F. Bejarano, A. Feeney, and M. Lucas, "A cymbal transducer for power ultrasonics applications," Sensors and Actuators A: Physical, vol. 210, no. April, pp. 182-189, Apr. 2014. [Online]. Available: http: //linkinghub.elsevier.com/retrieve/pii/S0924424714000880

[16] M. Nouh, O. Aldraihem, and a. Baz, "Energy Harvesting of Thermoacoustic-Piezo Systems With a Dynamic Magnifier," Journal of Vibration and Acoustics, vol. 134, no. 6, p. 061015, Oct. 2012. [Online]. Available: http://vibrationacoustics.asmedigitalcollection.asme.org/ article.aspx?doi $=10.1115 / 1.4005834$

[17] R. Murray and J. Rastegar, "Novel Two-Stage Piezoelectric-Based Ocean Wave Energy Harvesters for Moored or Unmoored Buoys," Active and Passive Smart Structures and Integrated Systems, vol. 7288, pp. $72880 \mathrm{E}-72880 \mathrm{E}-12$, Mar. 2009. [Online]. Available: http://proceedings.spiedigitallibrary.org/proceeding. aspx?articleid $=821922$

[18] N. S. Shenck, "A Demonstration of Useful Electric Energy Generation from Piezoceramics in a Shoe," Ph.D. dissertation, Massachusetts Institute of Technology, 1997. [Online]. Available: http://resenv.media.mit.edu/pubs/ theses/Nate-Thesis-Final.PDF

[19] J. Palosaari, M. Leinonen, J. Hannu, J. Juuti, and H. Jantunen, "Energy harvesting with a cymbal type piezoelectric transducer from low frequency compression," Journal of Electroceramics, vol. 28, no. 4, pp. 214-219, Mar. 2012. [Online]. Available: http://link.springer.com/10.1007/s10832-012-9713-8

[20] S. Priya and D. J. Inman, Energy harvesting technologies. Springer, 2008.

[21] J. Kymissis, C. Kendall, J. Paradiso, and N. Gershenfeld, "Parasitic power harvesting in shoes," Digest of Papers. Second International Symposium on Wearable Computers (Cat. No.98EX215), 1998.

[22] A. Pirisi, F. Grimaccia, M. Mussetta, and R. Zich, "Novel Speed Bumps Design and Optimization for Vehicles' Energy Recovery in Smart Cities," Energies, vol. 5, no. 12, pp. 4624-4642, Nov. 2012. [Online]. Available: http://www.mdpi.com/1996-1073/5/11/4624/

[23] D. Arnold, W. Kinsel, W. W. Clark, and C. Mo, "Exploration of New Cymbal Design in Energy Harvesting," Active and Passive Smart Structures 
and Integrated Systems, vol. 7977, pp. 1-6, 2011. [Online]. Available: http://proceedings.spiedigitallibrary.org/proceeding. aspx? articleid $=729212$

[24] Y. Jiangbo, S. Xiaobiao, X. Tao, and C. Weishan, "Modeling and Improvement of a Cymbal Transducer in Energy Harvesting," Journal of Intelligent Material Systems and Structures, vol. 21, no. 8, pp. 765-771, Mar. 2010. [Online]. Available: http://jim.sagepub.com/cgi/doi/10.1177/1045389X10366318

[25] C. Mo, D. Arnold, W. C. Kinsel, and W. W. Clark, "Modeling and experimental validation of unimorph piezoelectric cymbal design in energy harvesting," Journal of Intelligent Material Systems and Structures, vol. 24, no. 7, pp. 828-836, Nov. 2012. [Online]. Available: http: //jim.sagepub.com/cgi/doi/10.1177/1045389X12463459

[26] T. Purviance, S. Wickler, K. Clayson, T. Barnes, and C. Mo, "Development of low-profile piezoelectric energy harvester for high load application," in Technologies for Sustainability (SusTech), 2013 1st IEEE Conference on. IEEE, 2013, pp. $120-125$.

[27] P. Materials, "Piezoelectric materials," Electronics and Power, vol. 21, p. 53, 1975.

[28] Ltd Apc International, Piezoelectric Ceramics. Mackyville: American Piezo Ceramis International, 2011.

[29] R. Ballas, G., Piezoelectric Multilayer Beam Bending Actuators: Static and Dynamic Behavior and Aspects of Sensor Integration. Springer London, Limited, 2007.

[30] J. W. Morris, "Notes on the Thermodynamics of Solids Chapter 15: Tensors and Tensor Properties," pp. 274-328, 2008. [Online]. Available: http://www.mse. berkeley.edu/groups/morris/MSE201/Lecture_Notes/15.tensorproperties.pdf

[31] Noliac Group, "Noliac Ceramics NCE datasheet," p. 12. [Online]. Available: http://www.noliac.com/Files/Billeder/02Standard/Ceramics/Noliac _ CEramics_NCE_datasheet.pdf

[32] C. Benham, P.P. and Crawford, R.J. and Armstrong, Mechanics of Engineering Materials. Longman Group, 1996.

[33] J. Dicken, P. D. Mitcheson, I. Stoianov, and E. M. Yeatman, "Power-extraction circuits for piezoelectric energy harvesters in miniature and low-power applications," IEEE Transactions on Power Electronics, vol. 27, pp. 4514-4529, 2012.

[34] G. D. Szarka, B. H. Stark, and S. G. Burrow, "Review of power conditioning for kinetic energy harvesting systems," IEEE Transactions on Power Electronics, vol. 27, no. 2, pp. 803-815, 2012. 
[35] E. A. Grace, S. E. Rajan, and A. C. Asis, "Performance evaluation of different rectifiers for Piezo-electric energy harvesting applications," 2011 International Conference on Recent Advancements in Electrical, Electronics and Control Engineering, pp. 248-252, Dec. 2011. [Online]. Available: http://ieeexplore.ieee.org/lpdocs / epic03/wrapper.htm?arnumber $=6129810$

[36] E. Lefeuvre, "Piezoelectric Energy Harvesting Device Optimization by Synchronous Electric Charge Extraction," Journal of Intelligent Material Systems and Structures, vol. 16, no. 10, pp. 865-876, Oct. 2005. [Online]. Available: http://jim.sagepub.com/cgi/doi/10.1177/1045389X05056859

[37] a. Badel, "Efficiency Enhancement of a Piezoelectric Energy Harvesting Device in Pulsed Operation by Synchronous Charge Inversion," Journal of Intelligent Material Systems and Structures, vol. 16, no. 10, pp. 889-901, Oct. 2005. [Online]. Available: http://jim.sagepub.com/cgi/doi/10.1177/1045389X05053150

[38] H. Zhao, J. Ling, and J. Yu, "A comparative analysis of piezoelectric transducers for harvesting energy from asphalt pavement," Journal of the Ceramic Society of Japan, vol. 120, pp. 317-323, 2012.

[39] Ministry of Transportation, "Provincial Highways Traffic Volumes 2010," Tech. Rep., 2010. [Online]. Available: http://www.raqsb.mto. gov.on.ca/techpubs/TrafficVolumes.nsf/fa027808647879788525708a004b5df8/ f51986ea499a13b08525745f006dd30b/\protect \T1 \textdollarFILE/ ProvincialHighwaysTrafficVolumes2010AADTOnly.pdf

[40] MicroStrain, "Powering an EH-Link with piezoelectric source," p. 12, 2011. [Online]. Available: http://files.microstrain.com/tech_notes/PZT_TN_Rev0. 2.pdf

[41] A. Dogan, "Flextensional" moonie and Cymbal" Actuators," Ph.D. dissertation, Pennsylvania State University., 1994.

[42] A. Dogan and E. Uzgur, "Size and Material Effects on Cymbal Transducer for Actuator Applications," Ferroelectrics, vol. 331, no. 1, pp. 53-63, Mar. 2006. [Online]. Available: http://www.tandfonline.com/doi/abs/10.1080/ 00150190600734955

[43] Y. Ke, T. Guo, and J. Li, "A new-style, slotted-cymbal transducer with large displacement and high energy transmission," IEEE Transactions on Ultrasonics, Ferroelectrics, and Frequency Control, vol. 51, no. 9, pp. 1171-1177, 2004.

[44] M. Narayanan and R. W. Schwartz, "Design, fabrication and finite element modeling of a new wagon wheel flextensional transducer," Journal of Electroceramics, vol. 24, no. 3, pp. 205-213, Dec. 2008. [Online]. Available: http://link.springer.com/10.1007/s10832-008-9559-2 
[45] F. Wolf, a. Sutor, S. J. Rupitsch, and R. Lerch, "Modeling and measurement of influence of mechanical prestress on hysteresis of ferroelectric actuators," Procedia Engineering, vol. 25, pp. 1613-1616, 2011. [Online]. Available: http://dx.doi.org/10.1016/j.proeng.2011.12.399

[46] H. W. Kim, S. Priya, K. Uchino, and R. E. Newnham, "Piezoelectric energy harvesting under high pre-stressed cyclic vibrations," Journal of Electroceramics, vol. 15, pp. 27-34, 2005.

[47] A. Badel, A. Benayad, E. Lefeuvre, L. Lebrun, C. Richard, and D. Guyomar, "Single crystals and nonlinear process for outstanding vibration-powered electrical generators," IEEE Transactions on Ultrasonics, Ferroelectrics, and Frequency Control, vol. 53, no. 4, pp. 673-683, 2006.

[48] McMaster-Carr, "More About Stainless Steel Alloys," p. 2, 2010. [Online]. Available: http://www.mcmaster.com/

[49] K. Uchino, Ferroelectric Devices, J. Giniewicz, Ed. New York, Basel, Hong Kong: CRC Press, 2000.

[50] N. Putthongchai, T. Kulworawanichpong, P. Laoratanakul, and S. Srilomsak, "Effects of Composition and Diameter of PZT Transducers on Energy Harvesting," Integrated Ferroelectrics, vol. 114, no. 1, pp. 81-91, Nov. 2010. [Online]. Available: http://www.tandfonline.com/doi/abs/10.1080/10584587. 2010.488604

[51] Fujifilm, "Prescale," 2015. [Online]. Available: http://www.fujifilm.com/ products/prescale/prescalefilm/\#See_All 